\title{
An asymptotic preserving method for the linear transport equation on general meshes
}

\author{
Pierre Anguill ${ }^{\mathrm{a}}$, Patricia Cargo ${ }^{\mathrm{a}}$, Cedric Énaux ${ }^{\mathrm{a}}$, Philippe $\mathrm{Hoch}^{\mathrm{a}}$, \\ Emmanuel Labourasse ${ }^{\mathrm{a}, \mathrm{b}, *}$, Gerald Samba ${ }^{\mathrm{a}}$ \\ ${ }^{a} C E A, D A M, D I F, F-91297$ Arpajon, France. \\ ${ }^{b}$ Université Paris-Saclay, CEA DAM DIF, Laboratoire en Informatique Haute Performance pour \\ le Calcul et la simulation, 91297 Arpajon, France.
}

\begin{abstract}
While many numerical methods for the linear transport equation are available in the literature in 1D or on Cartesian meshes, fewer works are dedicated to the resolution of this model on unstructured meshes. In the context of radiative hydrodynamics, we need a method capable to handle a wide range of radiation regimes going from freestreaming to diffusion and to be coupled with a Lagrangian hydrodynamics solver. In this paper we design a method based on the micro-macro paradigm and to the Discrete Ordinates $\left(S_{N}\right)$ angular discretization, which fulfills these requirements. It allows to choose the limit transport scheme and the limit diffusion scheme. It is compared on challenging test problems to a Discontinuous Finite Element (DFE) method.
\end{abstract}

Keywords:

Finite Volume; radiative transfer; asymptotic analysis; computational transport; monotone anistotropic diffusion; unstructured meshes.

\section{Introduction}

In this paper, we are interested in the transport of photons in astrophysical phenomena or in Inertial Confinement Fusion experiment (refer to [24, 59, 71] for

\footnotetext{
${ }^{*}$ Corresponding author

Email addresses: pierre.anguill@cea.fr (Pierre Anguill), patricia.cargo@cea.fr (Patricia Cargo), cedric.enaux@cea.fr (Cedric Énaux), philippe.hoch@cea.fr

(Philippe Hoch), emmanuel.labourasse@cea.fr (Emmanuel Labourasse), gerald.samba@cea.fr (Gerald Samba)
} 
an overview of radiation driven physics). To this end, we consider the grey radiative transfer equations [59]

$$
\left\{\begin{array}{l}
\frac{1}{c} \partial_{t} I+\boldsymbol{\omega} \cdot \nabla I+\sigma I=\sigma B\left(T_{M}\right) \\
\partial_{t} E_{M}=c \sigma\left(E_{r}-a T_{M}^{4}\right)
\end{array}\right.
$$

where $c$ is the speed of light, $I$ denotes the grey radiative intensity, $\sigma$ the cross section, $B$ is the Planck function at the matter temperature $T_{M}, \boldsymbol{\omega}$ the direction, $E_{M}$ the matter energy density, and $E_{r}$ the radiative energy density. One of the 5 problem encountered when dealing with these equations is that they are non-linear and coupled. One solution to deal with this difficulty is the linearization used by Fleck and Cummings in [36]. This brings us, at each time iteration $n$, to the following linear problem on the time interval $\left[t^{n}, t^{n}+\Delta t\right]$

$$
\left\{\begin{array}{l}
\frac{1}{c} \partial_{t} I+\boldsymbol{\omega} \cdot \nabla I+\sigma^{n} I=f^{n} \sigma^{n} B^{n}+\left(1-f^{n}\right) \sigma^{n}<I> \\
\partial_{t} E_{M}=f^{n} c \sigma^{n}\left(E_{r}-\Phi^{n}\right)
\end{array}\right.
$$

where $\langle\cdot\rangle$ accounts for the angular mean value, $f=1 /(1+\beta c \sigma \Delta t)$ denotes the factor of Fleck, $\beta=\frac{d \Phi}{d E_{M}}, \Phi=a T_{M}^{4}$, and the superscript $n$ denotes quantities taken at time $t^{n}$.

The System (1) involves very different length and time scales. These scales are related to the scaled mean free path of the photons, called the Knudsen number. For Knudsen numbers close to unity, the transport of photons can be modeled by a hyperbolic kinetic equation. However, as the Knudsen number tends to zero (for instance in opaque media), it is well-known (refer for instance to [8]) that this equation tends to a diffusion equation (that is of parabolic type). That poses major difficulties for the numerical simulation, since a standard discretization constrains the time and space step proportionally to the Knudsen number. One way to circumvent this problem, is to decompose the calculation domain into a diffusive domain (small Knudsen number) and a transport domain (large Knudsen number), as explained for instance in $[7,32,31,38,40,48,53]$. Unfortunately, this solution is complicated to apply in our case, because the boundaries between diffusion and transport are fuzzy and moving, due to ablation processes. The alternative is to design numerical schemes able to handle all the radiative regimes without loss of accuracy or too drastic time-step restriction. These type of scheme is called Asymptotic Preserving (AP), and has been the subject of a considerable amount of work since the seminal work of Larsen et al [51, 52] (refer also to Jin and Levermore [46], who introduced the expression Asymptotic Preserving). A comprehensive description of the work that has since been done in this area is beyond the scope of this paper, and we invite 
the interested reader to refer to the review papers [44, 45]. Most of the numerical methods described in these papers are dedicated to $1 \mathrm{D}$ calculation or restricted to Cartesian meshes. However, there are relatively few numerical schemes capable of taking into account general meshes in multi-D. Early works in this field are based on a Discontinuous Finite Element (DFE) discretization [2, 3, 5, 6, 25, 65], which has been proven to capture the diffusive regime by construction. Another method [18] uses a Treffz Discontinuous Galerkin basis. Some recent works are based on Finite Volume schemes with an approximate Riemann solver. For instance, Franck and coGosse [39] to the nodal approximate Riemann solver of the Lagrangian Glace hydrodynamic scheme $[22,57]$. Another step in this direction was achieved by Berthon, Turpault and co-authors $[10,11,12,13]$, based on the HLL approximate Riemann solver (see for instance [69]). In [42], an AP scheme based on the DDFV formalism [41] is proposed.

Since we want to couple our radiation solver with a Lagrangian hydrodynamic scheme for the Euler system (as $[19,22,56,70]$ ), we desire our solver to fulfill the following properties:

P1 to be consistent on general meshes (meaning any polytopal valid mesh),

P2 to enforce conservation of radiative energy,

P3 to be able to handle all the radiation regimes from free-streaming to diffusion (Asymptotic Preserving),

$\mathbf{P} 4$ to have the radiative energy degrees of freedom located at the centers of the elements to ensure the compatibility with the hydrodynamic scheme.

Moreover, one of the major drawback of the Discrete Finite Element method on general meshes, is their algorithmic cost in the diffusion regime. While they are indeed Asymptotic Preserving, in the sense that the accuracy of the result and the time-step are independent of the Knudsen number, the CPU consumption strongly depends on acceleration technics as DSA (Diffusion Synthetic Acceleration) [4] or Transport Synthetic Acceleration (TSA) [63]. The development of these methods is still a field of research. This is why we want to free ourselves from these algorithms, and even be able

P5 to choose the diffusion scheme towards which our method tends when the Knudsen number becomes low. 
To our knowledge, no existing scheme fulfills all these requirements. That is why we were interested in designing a new scheme. To achieve this, we were inspired by a very popular method in the AP community, called micro-macro formulation. This method was introduced in [49] and popularized by Lemou and co-authors [9, 54]. It consists in decomposing the unknown (the radiative intensity in our case) into an equilibrium part (the angular mean value) and a deviation. Since the radiation is isotropic (does not depend on angle) in the diffusion limit, the deviation part tends to zero with the Knudsen number. This principle has been widely used for designing numerical AP schemes during the past two decades ([27, 29, 34, 47, 50,64] among others), and has similarities with some earlier methods called Variable Eddington to general meshes has been achieved. It may be because the limit diffusion scheme arising from the discretization proposed in [54] is a two-point flux approximation, which is known to be inconsistent on most of the meshes (refer for instance to [35]). In this paper, we propose a micro-macro scheme for the linear transport equation which can be proven to fulfill the propositions $\mathbf{P} \mathbf{1}$ to $\mathbf{P} 5$. To achieve this, we first perform the angular semi-discretization of the transport equation, using a Discrete Ordinate method. Then we perform the micro-macro decomposition, and obtain a transport equation for the angular dependant variables (deviation), and a diffusion equation for the angular main value (Section 2). The spatial and semi-implicit temporal discretization are described in Section 3. It is shown that our temporal discretization permits to choose any diffusion scheme for the main angular value. It allows us to choose a diffusion scheme which preserves the positivity of the energy $e$, which is an important property, especially in this regime (in which radiation is strongly coupled with the matter temperature). Finally, we assess the new method on several test problems in a wide range of regime, and compare it either to analytical solutions (if they exist) or to Discrete Finite Element method results in Section 5.

\section{Problem and properties}

\subsection{Continuous transport equation}

We focus on the following linear integro-differential transport equation, which is indeed of the form of Eq. (1)

$$
\frac{1}{v} \partial_{t} u(t, \boldsymbol{x}, \boldsymbol{\omega})+\boldsymbol{\omega} \cdot \nabla u(t, \boldsymbol{x}, \boldsymbol{\omega})+\sigma_{t} u(t, \boldsymbol{x}, \boldsymbol{\omega})=q(t, \boldsymbol{x}, \boldsymbol{\omega})+\sigma_{s} \int_{S^{2}} u\left(t, \boldsymbol{x}, \boldsymbol{\omega}^{\prime}\right) \frac{d \boldsymbol{\omega}^{\prime}}{4 \pi},
$$

where 
- $\boldsymbol{x} \in \Omega$ is the space coordinates in the open domain $\Omega \subset \mathbb{R}^{d}$, with $d$ the dimension of the problem;

- $t \in \mathbb{R}^{+}$is the time;

- $u(t, \boldsymbol{x}, \boldsymbol{\omega}) \in \mathbb{R}$ the distribution function;

- $v \in \mathbb{R}^{+}$is the norm of the velocity of the particles (equal to the speed of light in the case of photons);

- $\boldsymbol{\omega} \in S^{2}$ accounts for the direction of the velocity of the particles, $S^{2}$ being the unit sphere;

- $\sigma_{t}=\sigma_{a}+\sigma_{s}$, with $\sigma_{t}, \sigma_{a}$ and $\sigma_{s}$ respectively the total, absorption, and scattering cross sections;

- $q(t, \boldsymbol{x}, \boldsymbol{\omega})$ is a given source term.

We introduce the two first moments of $u$ with respect to $\boldsymbol{\omega}$

$$
e=<u>=\int_{S^{2}} u\left(\boldsymbol{\omega}^{\prime}\right) \frac{d \boldsymbol{\omega}^{\prime}}{4 \pi}, \quad \boldsymbol{f}=\int_{S^{2}} \boldsymbol{\omega}^{\prime} u\left(\boldsymbol{\omega}^{\prime}\right) \frac{d \boldsymbol{\omega}^{\prime}}{4 \pi} .
$$

By averaging Eq. (2) over all directions $\boldsymbol{\omega}$, we obtain a balance equation satisfied by $e$

$$
\frac{1}{v} \partial_{t} e+\nabla \cdot \boldsymbol{f}+\sigma_{a} e=s,
$$

with the isotropic part of the source

$$
s(t, \boldsymbol{x})=\int_{S^{2}} q\left(\boldsymbol{\omega}^{\prime}\right) \frac{\boldsymbol{d} \boldsymbol{\omega}^{\prime}}{4 \pi} .
$$

It is shown in [23] that if $q>0, \sigma_{a}>0$ and $\sigma_{s}>0$, Eq. (2) satisfies positivity. Moreover, in [30], we have that if $\sigma_{a}=0$, and $q=0, u$ and $e$ satisfies a conservation law and verify a global maximum principle $\left(u_{\min } \leq u \leq u_{\max }\right.$ and $e_{\min } \leq e \leq$ $e_{\max }$, where $u_{\min }, u_{\max }, e_{\min }, e_{\max }$ depend only on the initial state and the boundary conditions). It is well known (refer for instance to [24, 59]), that Eq. (2) satisfies a diffusion limit, in thick media, in the sense that when $\sigma_{s} \rightarrow \infty, \sigma_{a} \rightarrow 0$ and $q \rightarrow 0$, up to $\mathcal{O}\left(\frac{1}{\sigma_{s}^{2}}\right)$ :

$$
u=e-\frac{1}{\sigma_{s}} \boldsymbol{\omega} \cdot \boldsymbol{\nabla} e, \quad \boldsymbol{f}=-\frac{1}{3 \sigma_{s}} \boldsymbol{\nabla} e \quad \text { and } \quad \frac{1}{v} \partial_{t} e=\boldsymbol{\nabla} \cdot\left(\frac{1}{3 \sigma_{s}} \boldsymbol{\nabla} e\right) .
$$




\subsection{Semi-discrete angular equation}

We introduce $K>0$ angular directions, noted $\boldsymbol{\omega}_{k}$. Each direction is weighted by a positive weight $w_{k}$. Following the recommendations of $[20,21]$, we impose on the couples $\left(w_{k}, \boldsymbol{\omega}_{k}\right)$ the following constraints:

$$
\left\{\begin{array}{l}
\sum_{k=1}^{K} w_{k}=1, \\
\sum_{k=1}^{K} w_{k} \boldsymbol{\omega}_{k}=\mathbf{0}, \\
\sum_{k=1}^{K} w_{k} \boldsymbol{\omega}_{k} \otimes \boldsymbol{\omega}_{k}=\frac{1}{3} \overline{\mathbf{I}}_{K} .
\end{array}\right.
$$

This leads to an even number of directions, opposite in pairs and of equal weights $1 / K$. This quadrature is called quadrature Equal Weights [20]. It is then sufficient to discretize the sphere uniformly and then apply a renormalization to all the discrete directions to restore the coefficient $1 / 3$ of the moment of order 2.In this work, we have chosen to use a simple uniform angular grid which is controlled by its number

of degrees of freedom $N \geq 1$, and yields a number of discrete ordinates $K=4 N^{2}$ (in $2 \mathrm{D})$. Thus, with $u_{k} \equiv u\left(t, \boldsymbol{x}, \boldsymbol{\omega}_{k}\right)$, we are able to write the $K$ semi-discrete equations associated to the transport equation (2):

$$
\frac{1}{v} \partial_{t} u_{k}+\omega_{k} \cdot \nabla u_{k}+\sigma_{t} u_{k}=q_{k}+\sigma_{s} \sum_{k^{\prime}=1}^{K} u_{k^{\prime}} w_{k^{\prime}}
$$

It yields the semi-discrete formula for $e, f$ and $s$ :

$$
e(t, \boldsymbol{x}) \approx \sum_{k=1}^{K} w_{k} u_{k}, \quad \mathbf{f}(t, \boldsymbol{x}) \approx \sum_{k=1}^{K} w_{k} u_{k} \boldsymbol{\omega}_{k} \quad \text { and } \quad s(t, \boldsymbol{x}) \approx \sum_{k=1}^{K} w_{k} q_{k} .
$$

Likewise, the semi-discrete diffusion limit comes down to:

$$
\begin{gathered}
\forall t, \text { when } \sigma_{s} \rightarrow \infty, \sigma_{a} \rightarrow 0 \text { and } q \rightarrow 0, \text { and up to } \mathcal{O}\left(\frac{1}{\sigma_{s}^{2}}\right), \\
u_{k}=e-\frac{1}{\sigma_{s}} \boldsymbol{\omega}_{k} \cdot \nabla e(\forall k), \quad \boldsymbol{f}=-\frac{1}{3 \sigma_{s}} \nabla e, \quad \frac{1}{v} \partial_{t} e=\boldsymbol{\nabla} \cdot\left(\frac{1}{3 \sigma_{s}} \nabla e\right) .
\end{gathered}
$$




\subsection{Semi-discrete micro-macro formulation}

In order to have an Asymptotic-Preserving scheme, and to clearly distinguish between transport terms and diffusion terms, we introduce $\delta u_{k} \equiv u_{k}-e$, the deviation to isotropy.

By substracting (4) to (7), we obtain:

$$
\frac{1}{v} \partial_{t} \delta u_{k}+\boldsymbol{\omega}_{k} \cdot \nabla \delta u_{k}+\sigma_{t} \delta u_{k}=\boldsymbol{\nabla} \cdot \mathbf{f}-\boldsymbol{\omega}_{k} \cdot \nabla e+\delta q_{k},
$$

where $\delta q_{k} \equiv q_{k}-s$.

Thus, the first two angular moments of $\delta u_{k}$ are:

$$
\sum_{k=1}^{K} w_{k} \delta u_{k}=0, \quad \sum_{k=1}^{K} w_{k} \delta u_{k} \boldsymbol{\omega}_{k}=\boldsymbol{f} .
$$

It leaves unchanged the balance law for $e$ (4) except for the definition (9) of $\boldsymbol{f}$ as a function of the $\delta u_{k}$. Since no further approximation has been made, we still have the semi-discrete diffusion limit, hence:

$$
\begin{gathered}
\text { when } \sigma_{s} \rightarrow \infty, \sigma_{a} \rightarrow 0 \text { and } q_{k} \rightarrow 0, \text { and up to } \mathcal{O}\left(\frac{1}{\sigma_{s}^{2}}\right), \\
\delta u_{k}=-\frac{1}{\sigma_{s}} \boldsymbol{\omega}_{k} \cdot \nabla e(\forall k), \quad \boldsymbol{f}=-\frac{1}{3 \sigma_{s}} \nabla e, \quad \frac{1}{v} \partial_{t} e=\boldsymbol{\nabla} \cdot\left(\frac{1}{3 \sigma_{s}} \nabla e\right) .
\end{gathered}
$$

\section{2D planar spatio-temporal discretization on unstructured mesh}

We aim at solving the system (4)-(8) with the closure relations (9), on a 2D unstructured mesh. Since our ambition is to couple it with Lagrangian hydro schemes as $[22,56,70]$ (even if it is out of the scope of this paper), the geometric quality of the mesh is not controlled. This is why our discretization choices are guided by the concern of robustness. The conservation property of $e$ is very important for the targeted applications and has to be preserved. Moreover, we want our numerical method to be able to deal with all range of radiation regime, going from pure transport to diffusion. We give ourselves a regular mesh $\mathcal{M}$ of $\Omega$. We consider here that $\mathcal{M}$ is defined by a finite collection of cells $j$ that partition $\Omega(t)$. Specifically, we have

$$
\begin{aligned}
& \text { 1. } \forall j \in \mathcal{M}, \quad j \subset \Omega, \\
& \text { 2. } \forall \mathbf{x} \in \Omega, \quad \exists j \in \mathcal{M} \text { s.t. } \mathbf{x} \in \bar{j}, \\
& 3 . \forall j_{1}, j_{2} \in \mathcal{M}, \quad \overline{j_{1}} \cap \overline{j_{2}} \neq \emptyset \Longleftrightarrow \mid \begin{array}{l}
j_{1}=j_{2}, \\
\text { or } \\
\partial j_{1} \cap \partial j_{2} \neq \emptyset \text { and } j_{1} \cap j_{2}=\emptyset .
\end{array}
\end{aligned}
$$


The last relationship indicates that if two distinct cells intersect, it can only be through a piece of their edge (possibly a vertex). We define the following notations depicted on Fig. 1.

$\underline{\text { Notations }}$

- $j$ : cell index, and by extension the cell,

- $r$ : node index, and by extension the dual cell,

- $n$ : time iteration index,

- $p$ : optional sub-iteration index,

- $V_{j}$ : volume of cell $j$,

- $V_{r}$ : volume of dual cell $r$,

- $\boldsymbol{x}_{j}=\frac{1}{V_{j}} \int_{j} \boldsymbol{x}$ : center of cell $j$,

- $\boldsymbol{x}_{r}$ : position of node $r$,

- $\boldsymbol{x}_{j_{1} j_{2}}$ : vector equals to $\boldsymbol{x}_{j_{2}}-\boldsymbol{x}_{j_{1}}$,

- $l$ : edge of the primal cell $j$,

- $\tilde{l}$ : edge of the dual cell associated to a vertex $r$,

- $r(j)$ : designates a node $r$ of the cell $j$,

- $j(r)$ : designates a cell $j$ which owns the node $r$,

- $r^{\prime}(r)$ : designates a node $r^{\prime}$ which is adjacent to the node $r$,

- $l(j)$ : designates an edge $l$ which is contained by the cell $j$,

- $\boldsymbol{C}_{j r}:=\nabla_{\boldsymbol{x}_{r}} V_{j}$ : outgoing unit normal at node $r$ with respect to cell $j$,

160

- $\boldsymbol{C}_{r j}:=\nabla_{\boldsymbol{x}_{j}} V_{r}$ : outgoing unit normal at the center of the cell $j$ with respect to the dual cell $r$,

- $\boldsymbol{n}_{j l}$ : outgoing normal at edge $l$ with respect to the cell $j$,

- $\boldsymbol{n}_{r \tilde{l}}$ : outgoing normal at edge $\tilde{l}$ with respect to the dual cell $r$. 


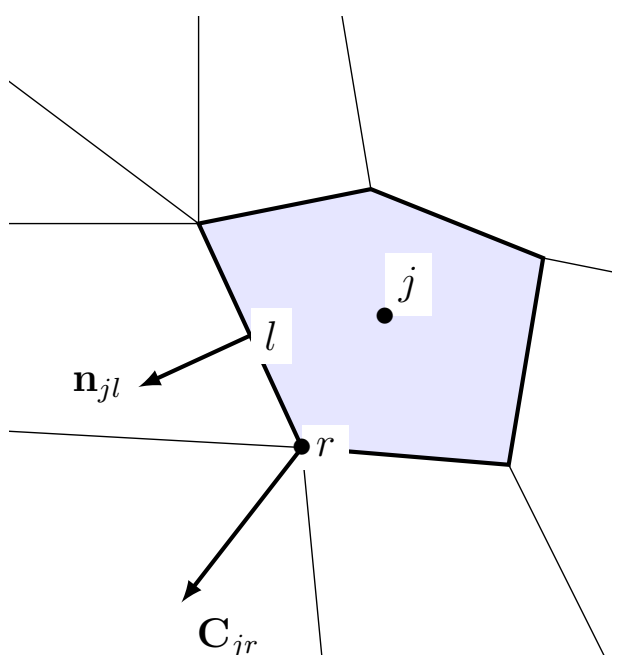

Figure 1: Notations (primal mesh)

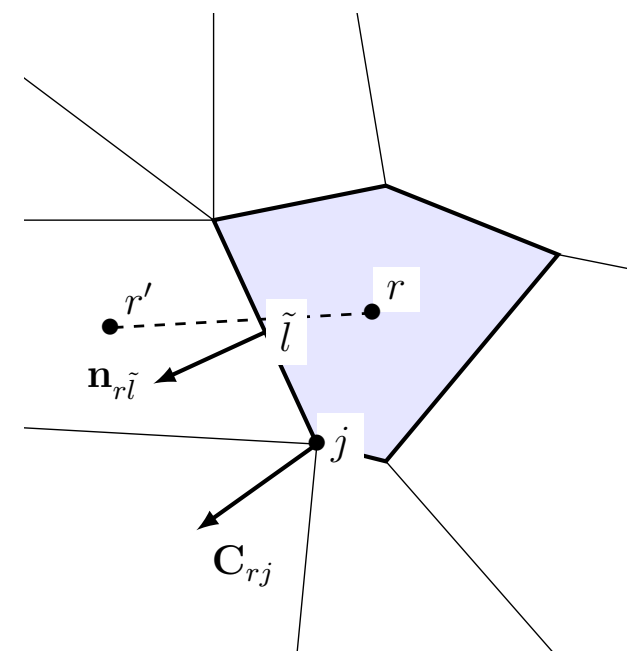

Figure 2: Notations (dual mesh)

We also need to define a dual mesh. It is built by joining the center of the cells $165 j$, and is depicted on Fig. 2.

We use a finite volume method for the spatial discretization. We integrate the Eq. (4) on the primal mesh, which gives

$$
\frac{1}{v} \partial_{t} \int_{j} e+\int_{j} \boldsymbol{\nabla} \cdot \boldsymbol{f}+\int_{j} \sigma_{a} e=\int_{j} s,
$$


We use the Green formula, a second-order accurate quadrature at nodes to approxi-

mate the boundary integrals, and define $\frac{1}{V_{j}} \int_{j} \varphi:=\varphi_{j}$, which yields

$$
V_{j} \frac{1}{v} \partial_{t} e_{j}+\sum_{r(j)} \boldsymbol{f}_{r} \cdot \boldsymbol{C}_{j r}+V_{j} \sigma_{a, j} e_{j}=V_{j} s_{j},
$$

With this quadrature formula, the flux $\boldsymbol{f}_{r}$ has to be evaluated at the nodes. As we saw previously, the angular discretization of $\boldsymbol{f}_{r}$, writes:

$$
\boldsymbol{f}_{r}=\sum_{k=1}^{K} w_{k} \delta u_{k, r} \boldsymbol{\omega}_{k}
$$

This is why we choice to perform a staggered discretization, and to writes the discrete balance of $\delta u_{k, r}$ at the nodes. Eq. (8) is then approximated with:

$$
\begin{gathered}
\frac{1}{v} \partial_{t} \delta u_{k, r}+\left[\boldsymbol{\omega}_{k} \cdot \boldsymbol{\nabla} \delta u_{k}\right]_{r}+\sigma_{t, r} \delta u_{k, r} \\
=\sum_{k^{\prime}=1}^{K} w_{k^{\prime}}\left[\boldsymbol{\omega}_{k^{\prime}} \cdot \boldsymbol{\nabla} \delta u_{k^{\prime}}\right]_{r}-\boldsymbol{\omega}_{k} \cdot[\boldsymbol{\nabla} e]_{r}+\delta q_{k, r} .
\end{gathered}
$$

The notation $[\varphi]_{r}$ accounts for a spatial discretization of $\varphi$ at the nodes, which is explained in the following.

Remark 3.1. Another possible choice of staggered discretization could be performed, in locating the $\delta u_{k}$ at the center of the edges instead at the nodes. In this case, the advection step would be performed on the diamond dual mesh, and the final global scheme would have the same properties than the one presented in this paper.

\subsection{Temporal discretization}

For this scheme, we use an implicit formulation. But, in order to avoid to solve a large linear system, we perform an hybrid time discretization, with sub-iterations.

The discrete version of Eq. (13) with this time discretization is:

$$
\begin{aligned}
& \frac{1}{v} \frac{\delta u_{k, r}^{n+1, p+1}-\delta u_{k, r}^{n}}{\Delta t}+\left[\boldsymbol{\omega}_{k} \cdot \boldsymbol{\nabla} \delta u_{k}\right]_{r}^{n+1, p+1 / 2}+\sigma_{t, r}^{n} \delta u_{k, r}^{n+1, p+1} \\
= & \sum_{k^{\prime}=1}^{K} w_{k^{\prime}}\left[\boldsymbol{\omega}_{k^{\prime}} \cdot \boldsymbol{\nabla} \delta u_{k^{\prime}}\right]_{r}^{n+1, p+1 / 2}-\boldsymbol{\omega}_{k} \cdot[\boldsymbol{\nabla} e]_{r}^{n+1, p+1}+\delta q_{k, r}^{n},
\end{aligned}
$$

where $n$ is the time index and $p$ the sub-iteration index. The index $p+1 / 2$ means that a part of discretization is performed at the sub-iteration $p$, while the other is 
performed at the iteration $p+1$ as explained in the following. The term $[\nabla e]_{r}^{n+1, p+1}$ is taken implicitly, which relies on an implicit diffusion equation on $e$, also explained in the following.

A very important property for this formulation to be meaningful is the following.

Proposition 3.1 (Preservation of the meaning of $e$ during time). With such a temporal discretization, $\sum_{k=1}^{K} w_{k} \delta u_{k, r}^{n}=0, \forall n, \quad \forall r$, whatever the nodal approximation of $\left[\boldsymbol{\omega}_{k} \cdot \boldsymbol{\nabla} \delta u_{k}\right]_{r}$ and $[\boldsymbol{\nabla} e]_{r}$ (see proof in Appendix I).

It means that $e$ indeed remains the discrete angular mean of $u_{k}$ with time.

The advection fluxes $\left[\boldsymbol{\omega}_{k} \cdot \boldsymbol{\nabla} \delta u_{k}\right]_{r}$ are discretized on the dual mesh, with an upwind implicit iterative scheme

$$
\begin{aligned}
{\left[\boldsymbol{\omega}_{k} \cdot \boldsymbol{\nabla} \delta u_{k}\right]_{r}^{n+1, p+1 / 2} } & =\frac{1}{V_{r}} \sum_{\tilde{l}, \boldsymbol{n}_{r \tilde{l}} \cdot \boldsymbol{\omega}_{k}>0}|\tilde{l}| \boldsymbol{n}_{r \tilde{l}} \cdot \boldsymbol{\omega}_{\boldsymbol{k}} \delta u_{k, r}^{n+1, p+1} \\
& +\frac{1}{V_{r}} \sum_{\tilde{l}, \boldsymbol{n}_{r \tilde{l}} \cdot \boldsymbol{\omega}_{k}<0}|\tilde{l}| \boldsymbol{n}_{r \tilde{l}} \cdot \boldsymbol{\omega}_{\boldsymbol{k}} \delta u_{k, r^{\prime}(r)}^{n+1, p},
\end{aligned}
$$

where $\tilde{l}$ is the edge intersecting the segment $\left[r r^{\prime}\right]$ joining the nodes $r$ and $r^{\prime}$ (two centers of the dual cells), see Fig. 2.

Proposition 3.2 (stability of the advection scheme). The advection scheme corresponding to (14) with the flux (15), and without source terms, is unconditionally stable (see proof in Appendix E).

It remains to discretize the term $\boldsymbol{\omega}_{k} \cdot[\boldsymbol{\nabla} e]_{r}^{n+1, p+1}$, to this end, we use the same nodal quadrature rule as before:

$$
\boldsymbol{\omega}_{k} \cdot[\nabla e]_{r}^{n+1, p+1} \equiv \frac{1}{V_{r}} \sum_{j(r)} e_{j}^{n+1, p+1} \boldsymbol{C}_{r j} \cdot \boldsymbol{\omega}_{k} .
$$

Thanks to our time discretization, we can provide an analytical expression for $\delta u_{k, r}^{n+1, p+1}$. Indeed, defining $\boldsymbol{\delta} \boldsymbol{u}_{r}$ as the vector whose $k$ th component is $\delta u_{k, r}$, the Eq. (14) can be put in the form of a $K \times K$ system, local to each node:

$$
\left(\overline{\overline{\mathbf{I}}}_{K}-\boldsymbol{a}_{r}^{n} \otimes \boldsymbol{b}_{r}^{n}\right) \boldsymbol{\delta} \boldsymbol{u}_{r}^{n+1, p+1}=\mathcal{A}_{r}^{n}+\mathcal{B}_{r}^{n+1, p}+\mathcal{C}_{r}^{n+1, p+1}
$$


where $\overline{\overline{\mathbf{I}}}_{K}$ is the identity matrix of rank $K, \boldsymbol{a}_{r}^{n}$ and $\boldsymbol{b}_{r}^{n}$ are the vectors of size $K$ of components

$$
\begin{aligned}
& a_{k, r}^{n}=1 /\left(1+v \Delta t \sigma_{t, r}^{n}+\frac{v \Delta t}{V_{r}} \sum_{\tilde{l}, \boldsymbol{n}_{r \tilde{l}} \cdot \boldsymbol{\omega}_{k}>0}|\tilde{l}| \boldsymbol{n}_{r \tilde{l}} \cdot \boldsymbol{\omega}_{\boldsymbol{k}}\right), \\
& b_{k, r}^{n}=w_{k} \frac{v \Delta t}{V_{r}} \sum_{\tilde{l},, \boldsymbol{n}_{r \tilde{l}} \cdot \boldsymbol{\omega}_{k}>0}|\tilde{l}| \boldsymbol{n}_{r \tilde{l}} \cdot \boldsymbol{\omega}_{\boldsymbol{k}} .
\end{aligned}
$$

and $\mathcal{A}_{r}^{n}, \mathcal{B}_{r}^{n+1, p}$ and $\mathcal{C}_{r}^{n+1, p+1}$ are the vectors of size $K$ defined by:

$$
\begin{aligned}
& \mathcal{A}_{k, r}^{n}=a_{k, r}^{n}\left(\delta u_{k, r}^{n}+v \Delta t \delta q_{k, r}^{n}\right) \\
& \mathcal{B}_{k, r}^{n+1, p}=a_{k, r}^{n} v \Delta t\left(\sum_{k^{\prime}=1}^{K} w_{k^{\prime}} \frac{1}{V_{r}} \sum_{\tilde{l}, \boldsymbol{n}_{r \tilde{l}} \cdot \boldsymbol{\omega}_{k^{\prime}}<0}|\tilde{l}| \boldsymbol{n}_{r \tilde{l}} \cdot \boldsymbol{\omega}_{k^{\prime}} \delta u_{k^{\prime}, r^{\prime}}^{n+1, p}\right.
\end{aligned}
$$

$$
\begin{aligned}
& \left.-\frac{1}{V_{r}} \sum_{\tilde{l}, \boldsymbol{n}_{r \tilde{l}} \cdot \boldsymbol{\omega}_{k}<0}|\tilde{l}| \boldsymbol{n}_{r \tilde{l}} \cdot \boldsymbol{\omega}_{\boldsymbol{k}} \delta u_{k, r^{\prime}}^{n+1, p}\right), \\
& \mathcal{C}_{k, r}^{n+1, p+1}=-a_{k, r}^{n} v \Delta t \boldsymbol{\omega}_{k} \cdot[\boldsymbol{\nabla} e]_{r}^{n+1, p+1} .
\end{aligned}
$$

Here, to prove the invertibility of the linear system (16), we use a corollary of the Sherman-Morrison lemma whose proof is provided in Appendix C. The statement of the corollary is: Let $\overline{\mathbf{I}}_{K}$ be the identity matrix of size $K \times K$, and let $\boldsymbol{a}$ and $\boldsymbol{b}$ be two vectors (columns) of size $K$. The matrix $\overline{\overline{\mathbf{I}}}_{K}-\boldsymbol{a} \otimes \boldsymbol{b}$ is invertible if $\boldsymbol{a} \cdot \boldsymbol{b} \neq 1$, and its inverse is:

$$
\left(\overline{\overline{\mathbf{I}}}_{K}-\boldsymbol{a} \otimes \boldsymbol{b}\right)^{-1}=\overline{\overline{\mathbf{I}}}_{K}+\frac{\boldsymbol{a} \otimes \boldsymbol{b}}{1-\boldsymbol{a} \cdot \boldsymbol{b}} .
$$

Using that $\sum_{k} w_{k}=1$, it is easy to show that $\boldsymbol{a}_{r}^{n} \cdot \boldsymbol{b}_{r}^{n}<1$, and consequently that the system (16) is invertible. Moreover, we are able to express its solution $\boldsymbol{\delta} \boldsymbol{u}_{r}^{n+1, p+1}$

$$
\boldsymbol{\delta} \boldsymbol{u}_{r}^{n+1, p+1}=\boldsymbol{\alpha}_{r}^{n}+\boldsymbol{\beta}_{r}^{n+1, p}+\boldsymbol{\gamma}_{r}^{n+1, p+1},
$$


where $\boldsymbol{\alpha}_{r}^{n}, \boldsymbol{\beta}_{r}^{n+1, p}$ and $\boldsymbol{\gamma}_{r}^{n+1, p+1}$ are the vectors of size $K$ defined by:

$$
\begin{aligned}
& \boldsymbol{\alpha}_{r}^{n}=\left(\overline{\overline{\mathbf{I}}}_{K}+\frac{\boldsymbol{a}_{r}^{n} \otimes \boldsymbol{b}_{r}^{n}}{1-\boldsymbol{a}_{r}^{n} \cdot \boldsymbol{b}_{r}^{n}}\right) \mathcal{A}_{r}^{n}, \\
& \boldsymbol{\beta}_{r}^{n+1, p}=\left(\overline{\overline{\mathbf{I}}}_{K}+\frac{\boldsymbol{a}_{r}^{n} \otimes \boldsymbol{b}_{r}^{n}}{1-\boldsymbol{a}_{r}^{n} \cdot \boldsymbol{b}_{r}^{n}}\right) \mathcal{B}_{r}^{n+1, p}, \\
& \boldsymbol{\gamma}_{r}^{n+1, p+1}=\left(\overline{\overline{\mathbf{I}}}_{K}+\frac{\boldsymbol{a}_{r}^{n} \otimes \boldsymbol{b}_{r}^{n}}{1-\boldsymbol{a}_{r}^{n} \cdot \boldsymbol{b}_{r}^{n}}\right) \mathcal{C}_{r}^{n+1, p+1} .
\end{aligned}
$$

The expression of $\gamma_{r}^{n+1, p+1}$ can be simplified thanks to the following argument. Recalling that the directions are opposite in pairs and of equal weights, meaning that $\forall k \in[1 ; K], \exists \bar{k} \in[1 ; K]$ s.t. $\left\{\bar{k} \neq k, w_{\bar{k}}=w_{k}, \boldsymbol{\omega}_{\bar{k}}=-\boldsymbol{\omega}_{k}\right\}$, we infer from Eq. (17) that $a_{\bar{k}, n}^{i}=a_{k, n}^{i}$ and $b_{\bar{k}, n}^{i}=b_{k, n}^{i}$, s.t. $\forall k$ :

$$
\gamma_{k, r}^{n+1, p+1}=-v \Delta t(a_{k, r}^{n} \boldsymbol{\omega}_{k}+a_{k, r}^{n} \underbrace{\sum_{k^{\prime}=1}^{K} \frac{a_{k^{\prime}, r}^{n} b_{k^{\prime}, r}^{n}}{1-\boldsymbol{a}_{r}^{n} \cdot \boldsymbol{b}_{r}^{n}} \boldsymbol{\omega}_{k^{\prime}}}_{\equiv \mathbf{0}}) \cdot[\boldsymbol{\nabla} e]_{r}^{n+1, p+1},
$$

which means that $\boldsymbol{\gamma}_{r}^{n+1, p+1}$ reduces to:

$$
\gamma_{r}^{n+1, p+1}=\mathcal{C}_{r}^{n+1, p+1}
$$

It gives the discrete approximate solution of Eq. (8).

It remains now to solve the Eq. (4). To avoid a drastic parabolic CFL, we perform an implicit backward Euler time discretization of (11):

$$
\frac{1}{v} \frac{e_{j}^{n+1}-e_{j}^{n}}{\Delta t}+\frac{1}{V_{j}} \sum_{r(j)} \boldsymbol{f}_{r}^{n+1} \cdot \boldsymbol{C}_{j r}+\sigma_{a, j}^{n} e_{j}^{n+1}=s_{j}^{n} .
$$

Since $\boldsymbol{f}_{r}^{n+1, p+1}=\sum_{k=1}^{K} w_{k} \delta u_{k, r}^{n+1, p+1} \boldsymbol{\omega}_{k}$, the expression (19) of $\delta u_{k, r}^{n+1, p+1}$ yields

$$
\begin{aligned}
\boldsymbol{f}_{r}^{n+1, p+1} & =\sum_{k=1}^{N} w_{k} \boldsymbol{\omega}_{k}\left(\alpha_{k, r}^{n}+\beta_{k, r}^{n+1, p}+\gamma_{k, r}^{n+1, p+1}\right) \\
& =\sum_{k=1}^{K} w_{k} \boldsymbol{\omega}_{k} \alpha_{k, r}^{n}+\sum_{k=1}^{K} w_{k} \boldsymbol{\omega}_{k} \beta_{k, r}^{n+1, p}-\overline{\bar{D}}_{r}^{n}[\nabla e]_{r}^{n+1, p+1}
\end{aligned}
$$


where $\overline{\overline{\boldsymbol{D}}}_{r}^{n}$ is an anisotropic tensor diffusion coefficient defined by

$$
\overline{\overline{\boldsymbol{D}}}_{r}^{n}=v \Delta t \sum_{k=1}^{K} w_{k} a_{k, r}^{n} \boldsymbol{\omega}_{k} \otimes \boldsymbol{\omega}_{k}
$$
fusion operator comes from the expression of $\boldsymbol{\gamma}_{r}^{n+1} \overline{\overline{\boldsymbol{D}}}_{r}^{n}[\boldsymbol{\nabla} e]_{r}^{n+1, p+1}$, it remains to define $\overline{\overline{\boldsymbol{D}}}_{l}^{n}$ from $\overline{\overline{\boldsymbol{D}}}_{r}^{n}$.

We have the important property for the tensor $\overline{\overline{\boldsymbol{D}}}_{r}^{n}$. positive (see proof in Appendix B). taken at the edges and not at the nodes:

Proposition 3.3 (definite positiveness of the diffusion tensor). $\overline{\overline{\boldsymbol{D}}}_{r}^{n}$ is definite

The term $\sum_{r(j)}\left(\overline{\overline{\boldsymbol{D}}}_{r}^{n}[\nabla e]_{r}^{n+1, p+1}\right) \cdot \boldsymbol{C}_{j r}$ in Eq. (24) is a discrete diffusion operator. However, it would lead to a matrix which is not unconditionally invertible, and a diffusion scheme which is not positive [37]. This is why we reformulate Eq. (24), in order to use the diffusion scheme of our choice, and for which the outgoing fluxes are

$$
\sum_{r(j)}\left(\overline{\overline{\boldsymbol{D}}}_{r}^{n}[\boldsymbol{\nabla} e]_{r}^{n+1, p+1}\right) \cdot \boldsymbol{C}_{j r} \approx \sum_{l(j)}|l|\left(\overline{\overline{\boldsymbol{D}}}_{l}^{n}[\boldsymbol{\nabla} e]_{l}^{n+1, p+1}\right) \cdot \boldsymbol{n}_{j l}
$$

where $[\nabla e]_{l}$ is the gradient of $e$ evaluated at the center of the edge $l$. This is a valid approximation since $\sum_{r(j)} \varphi_{r} \boldsymbol{C}_{j r}$ and $\sum_{l(j)}|l| \varphi_{l} \boldsymbol{n}_{j l}$ are two second-order accurate approximations of $\int_{\partial j} \varphi \boldsymbol{n}$ (we recall the proof in Appendix A). It is the subject of the following section to explain how we approximate $[\nabla e]_{l}^{n+1, p+1}$. Since, the diffusion operator comes from the expression of $\gamma_{r}^{n+1, p+1}$, and more particularly from 
In order to obtain such a discretization, we define, for each edge $l$ (defined by the nodes $r$ and $r^{\prime}$ )

$$
\overline{\overline{\boldsymbol{D}}}_{l}^{n}=\frac{1}{2} \overline{\overline{\boldsymbol{D}}}_{r}^{n}+\frac{1}{2} \overline{\overline{\boldsymbol{D}}}_{r^{\prime}}^{n}
$$

which is also a second-order accurate approximation. Thereby, we obtain a linear system on $e$ :

245

$$
\begin{aligned}
& \left(1+v \Delta t \sigma_{a, j}^{n}\right) e_{j}^{n+1, p+1}-\frac{v \Delta t}{V_{j}} \sum_{l(j)}|l|\left(\overline{\boldsymbol{D}}_{l}^{n}[\nabla]_{l}^{n+1, p+1}\right) \cdot \boldsymbol{n}_{j l} \\
& =e_{j}^{n}+v \Delta t s_{j}^{n}-\frac{v \Delta t}{V_{j}} \sum_{r(j)} \sum_{k=1}^{K} w_{k} \boldsymbol{\omega}_{k}\left(\alpha_{k, r}^{n}+\beta_{k, r}^{n+1, p}\right) \cdot \boldsymbol{C}_{j r}
\end{aligned}
$$

Finally, to complete the scheme, the last ingredient we need is the calculation of the diffusion operator:

$$
\frac{v \Delta t}{V_{j}} \sum_{l(j)}|l|\left(\overline{\overline{\boldsymbol{D}}}_{l}^{n}[\boldsymbol{\nabla} e]_{l}^{n+1, p+1}\right) \cdot \boldsymbol{n}_{j l} .
$$

To achieve that, we use an implicit solver for diffusion which is an extension of [14] to tensorial diffusion coefficient, and is presented in the following subsection.

\subsection{Diffusion}

Consider the following tensorial anisotropic diffusion problem :

$$
\begin{cases}\partial_{t} e-\boldsymbol{\nabla} \cdot(\overline{\overline{\boldsymbol{D}}} \boldsymbol{\nabla} e)=h & \text { in }(0, T) \times \Omega \\ e=g & \text { on }(0, T) \times \partial \Omega\end{cases}
$$

- $\Omega$ bounded open set of $\mathbb{R}^{2}$,

- $h \in L^{2}(\Omega)$,

- $g \in H^{1 / 2}(\partial \Omega)$,

- $\overline{\overline{\boldsymbol{D}}}:(0, T) \times \Omega \rightarrow \mathcal{M}_{2,2}$ is a bounded measurable function, s.t. $\overline{\overline{\boldsymbol{D}}}$ is a definite positive symmetric tensor,

- $e(t=0, \boldsymbol{x})=e_{0} \in H^{1}(\Omega)$ initial condition.

By integration of the equation in a cell $j$, we obtain

$$
\int_{j} \frac{\partial e}{\partial t}+\sum_{l \in \partial j}\left(-\int_{l} \boldsymbol{\nabla} e \cdot \tilde{\boldsymbol{n}}_{j l}\right)=\int_{j} h
$$


where $\tilde{\boldsymbol{n}}_{\boldsymbol{j} \boldsymbol{l}}=\overline{\overline{\boldsymbol{D}}}_{l} \boldsymbol{n}_{j l}$, with $\boldsymbol{n}_{j l}$ the outgoing normal (defined on previous section, refer to Fig. 1) to the edge $l$ of the cell $j$. We consider the flux (notations are given on Fig. 3)

$$
\mathcal{F}_{j, l}=-\int_{l} \nabla e \cdot \tilde{\boldsymbol{n}}_{j l}
$$

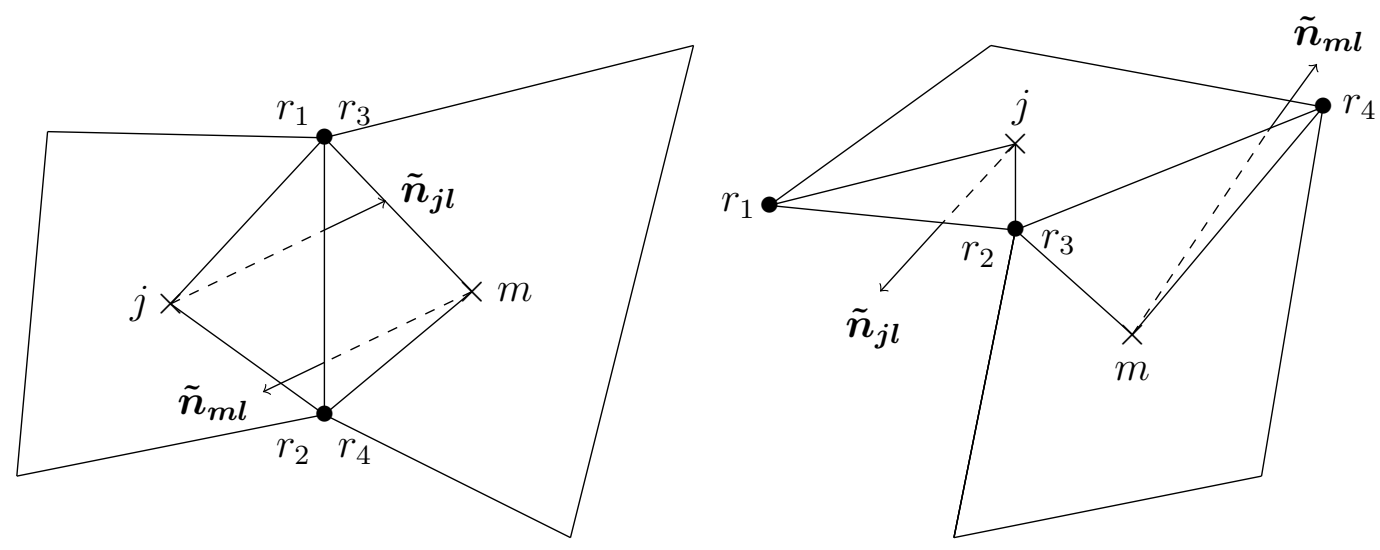

Figure 3: Expression of $\tilde{\boldsymbol{n}}_{\boldsymbol{j l}}$ and $\tilde{\boldsymbol{n}}_{\boldsymbol{m l}}$

Let us define $\boldsymbol{x}_{j r_{1}}$ and $\boldsymbol{x}_{j r_{2}}$ s.t. $\tilde{\boldsymbol{n}}_{\boldsymbol{j} \boldsymbol{l}}$ has non-negative coefficients when decomposed into the basis $\left(\boldsymbol{x}_{j r_{1}}, \boldsymbol{x}_{j r_{2}}\right)$ (refer to Fig. 3 for different examples). Then, we express $\tilde{\boldsymbol{n}}_{\boldsymbol{j} l}$ in the basis $\left(\boldsymbol{x}_{j r_{1}}, \boldsymbol{x}_{j r_{2}}\right)$

$$
\tilde{\boldsymbol{n}}_{\boldsymbol{j l}}=\lambda_{j} \frac{\boldsymbol{x}_{j r_{1}}}{\left\|\boldsymbol{x}_{j r_{1}}\right\|}+\eta_{j} \frac{\boldsymbol{x}_{j r_{2}}}{\left\|\boldsymbol{x}_{j r_{2}}\right\|}
$$

with $\lambda_{j} \in \mathbb{R}^{+}$and $\eta_{j} \in \mathbb{R}^{+}$. Inserting this decomposition into (26) yields

$$
\mathcal{F}_{j, l}=-\int_{l}\left(\lambda_{j} \nabla e \cdot \frac{\boldsymbol{x}_{j r_{1}}}{\left\|\boldsymbol{x}_{j r_{1}}\right\|}+\eta_{j} \nabla e \cdot \frac{\boldsymbol{x}_{j r_{2}}}{\left\|\boldsymbol{x}_{j r_{2}}\right\|}\right) .
$$

Thanks to a Taylor expansion, we have

$$
\nabla e \cdot \frac{\boldsymbol{x}_{j r_{i}}}{\left\|\boldsymbol{x}_{j r_{i}}\right\|}=\frac{e\left(\boldsymbol{x}_{r_{i}}\right)-e\left(\boldsymbol{x}_{j}\right)}{\left\|\boldsymbol{x}_{j r_{i}}\right\|}+O(\Delta x),
$$

and then,

$$
\mathcal{F}_{j, l}=-|l|\left(\lambda_{j} \frac{e_{r_{1}}-e_{j}}{\left\|\boldsymbol{x}_{j r_{1}}\right\|}+\eta_{j} \frac{e_{r_{2}}-e_{j}}{\left\|\boldsymbol{x}_{j r_{2}}\right\|}\right)+O\left(\Delta x^{2}\right)
$$


This last formulation involves $e_{r_{1}}$ and $e_{r_{2}}$ which are nodal evaluations of $e$ at the nodes $r_{i}$. To achieve the computation of $e$ at these nodes, we use a least-squares procedure as in [26]. We perform the same procedure for approximating the flux $\mathcal{F}_{m, l}$.

We can now define

$$
F_{1}=-|l|\left(\lambda_{j} \frac{e_{r_{1}}-e_{j}}{\left\|\boldsymbol{x}_{j r_{1}}\right\|}+\eta_{j} \frac{e_{r_{2}}-e_{j}}{\left\|\boldsymbol{x}_{j r_{2}}\right\|}\right) \text { and } F_{2}=-|l|\left(\lambda_{m} \frac{e_{r_{3}}-e_{m}}{\left\|\boldsymbol{x}_{m r_{3}}\right\|}+\eta_{m} \frac{e_{r_{4}}-e_{m}}{\left\|\boldsymbol{x}_{m r_{4}}\right\|}\right)
$$

which gives us two consistent approximations of the flux. It yields our expression of the flux from the cell $j$ to the cell $m$ as a convex combination of $F_{1}$ and $-F_{2}$

$$
F_{j, l}=\mu_{1}(e) F_{1}-\mu_{2}(e) F_{2}, \quad\left(=-F_{m, l}\right),
$$

with $\mu_{1}+\mu_{2}=1$. It leads to the following expression for our discrete flux $F_{j, l}$

$$
F_{j, l}=\mu_{1}|l|\left(\frac{\lambda_{j}}{\left\|\boldsymbol{x}_{j r_{1}}\right\|}+\frac{\eta_{j}}{\left\|\boldsymbol{x}_{j r_{2}}\right\|}\right) e_{j}-\mu_{2}|l|\left(\frac{\lambda_{m}}{\left\|\boldsymbol{x}_{m r_{3}}\right\|}+\frac{\eta_{m}}{\left\|\boldsymbol{x}_{m r_{4}}\right\|}\right) e_{m}+\mu_{1} R_{1}-\mu_{2} R_{2},
$$

where $R_{1}$ and $R_{2}$ depend respectively on $e_{r_{1}}, e_{r_{2}}$ and $e_{r_{3}}, e_{r_{4}}$. Choosing $\mu_{1}$ and $\mu_{2}$ as the solution of the following system

$$
\left\{\begin{array}{l}
\mu_{1}+\mu_{2}=1 \\
\mu_{1} R_{1}-\mu_{2} R_{2}=0
\end{array}\right.
$$

we end up with a two-point but non-linear approximation of the flux $\mathcal{F}_{j, l}$

$$
F_{j, l}=\mu_{1}|l|\left(\frac{\lambda_{j}}{\left\|\boldsymbol{x}_{j r_{1}}\right\|}+\frac{\eta_{j}}{\left\|\boldsymbol{x}_{j r_{2}}\right\|}\right) e_{j}-\mu_{2}|l|\left(\frac{\lambda_{m}}{\left\|\boldsymbol{x}_{m r_{3}}\right\|}+\frac{\eta_{m}}{\left\|\boldsymbol{x}_{m r_{4}}\right\|}\right) e_{m},
$$

since $\mu_{1}$ and $\mu_{2}$ now depend on $e$. We note that $\mu_{1}$ and $\mu_{2}$ are non-negative, provided that the nodal interpolations of $e$ are non-negative. To this end, we apply the same trick as in [14]. As we explain in the next subsection, this procedure enforces the positivity of the diffusion scheme. However, a fixed point algorithm is required to solve the non-linearity of the fluxes.

It remains to express the boundary conditions, for which we use the following approximation

$$
\boldsymbol{\nabla} e \cdot \boldsymbol{x}_{j t}=e_{j}-e_{t}+O(\Delta x)
$$

using $\lambda_{j} \boldsymbol{x}_{j t}=\overline{\overline{\boldsymbol{D}}}_{l} \boldsymbol{n}_{\boldsymbol{j}, l}=\tilde{n}_{j, l}$. With $e_{t}=g\left(\boldsymbol{x}_{t}\right)$, we have

$$
F_{j, l}=|l| \lambda_{j}\left(e_{j}-g\left(\boldsymbol{x}_{t}\right)\right) .
$$




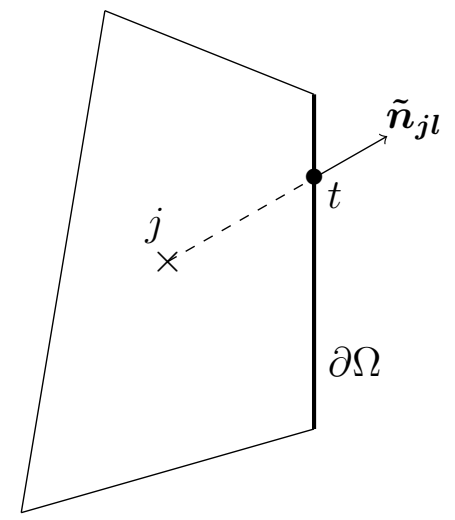

Figure 4: Boundary conditions notations

Finally, the implicit diffusion scheme is

$$
\left\{\begin{array}{l}
\frac{1}{\Delta t}\left(e^{n+1}-e^{n}\right)+M\left(e^{n+1}\right) e^{n+1}=h+g \\
e^{n+1} \geq 0
\end{array},\right.
$$

with

and

$$
\begin{gathered}
\Phi_{j, l}=\mu_{1}|l|\left(\frac{\lambda_{j}}{\left\|\boldsymbol{x}_{j r_{1}}\right\|}+\frac{\eta_{j}}{\left\|\boldsymbol{x}_{j r_{2}}\right\|}\right), \\
\Phi_{m, l}=\mu_{2}|l|\left(\frac{\lambda_{m}}{\left\|\boldsymbol{x}_{m r_{3}}\right\|}+\frac{\eta_{m}}{\left\|\boldsymbol{x}_{m r_{4}}\right\|}\right),
\end{gathered}
$$

$$
\left\{\begin{array}{l}
{[M(e)]_{j j}=\frac{1}{V_{j}}\left(\sum_{l \in \mathcal{M} \backslash \partial \Omega} \Phi_{j, l}+\sum_{l \in \mathcal{M} \cap \partial \Omega}|l| \lambda_{j}\right) .} \\
{[M(e)]_{j m}=-\frac{\Phi_{m, l}}{V_{j}} \text { if } j \neq m}
\end{array} .\right.
$$

\subsection{Properties of the diffusion scheme and of the global scheme}

The diffusion scheme fulfills the following properties.

Proposition 3.4 (Positivity (monotony) of the diffusion scheme). The diffusion scheme is positive and is unconditionnaly stable.

This property is also called monotony in other publications [55,60,68]. It means that if $e^{n} \geq 0, h \geq 0$ and $g \geq 0$, then $e^{n+1} \geq 0$. The proof is displayed in Appendix G. 
Proposition 3.5 (Convergence). Providing that $e^{n} \geq 0, h \geq 0$ and $g \geq 0$, the scheme has a unique solution, and the fixed-point strategy can be proven to converge under a parabolic CFL restriction.

The proof can be found in [14].

Proposition 3.6 (Conservation of the diffusion scheme). The diffusion scheme is conservative.

See the proof in Appendix F.

We are now able to demonstrate the following property on the global balance of energy (25).

Proposition 3.7 (Conservation of the global scheme). The global scheme is conservative.

See the proof in Appendix $\mathrm{H}$.

We finally prove that the global scheme is Asymptotic Preserving.

Proposition 3.8 (Asymptotic Preserving behaviour of the scheme). In the limit

$$
v \Delta t \sigma_{t, r}^{n}>>1, \quad v \Delta t \sigma_{a, j}^{n}<<1, \quad v \Delta t\left|s_{j}^{n}\right|<<e_{j}^{n}, \text { and } v \Delta t\left|q_{k, r}^{n}\right|<<\left|\delta_{k, r}^{n}\right|,
$$

the system (14)-(25) tends to the following discrete implicit diffusion operator

$$
e^{n+1}-e^{n}=\Delta t \overline{\bar{M}} e^{n+1}
$$

where $\boldsymbol{e} \in \mathbb{R}^{\# j}$ is the vector of components $e_{j}$ and of size the number of cells \#j, and $\overline{\overline{\boldsymbol{M}}} \in \mathbb{R}^{\# j} \times \mathbb{R}^{\# j}$ is the diffusion matrix corresponding to the discretization of $\sum_{l(j)}|l|\left(\frac{1}{3 \sigma_{s, j}}[\nabla e]_{l}^{n+1}\right) \cdot \boldsymbol{n}_{j l}$ with the algorithm described in this section.

See the proof in Appendix J.

Remark 3.2 (About the Asymptotic Preserving property). Despite that the transport scheme used to solve Eq. (8) is a simple upwind discretization, the global scheme is Asymptotic Preserving. This is due to the fact that all the $\delta u_{k}$ tends to zero in the diffusive regime, and then only the equation on e matters. 


\section{Summary of the scheme}

Time iterations $(n)$ :

- calculation of $\boldsymbol{a}_{r}^{n}, \boldsymbol{b}_{r}^{n}$, and $\boldsymbol{\alpha}_{r}^{n}$, with Eq. (17), (18) and (20),

300

305

- Do (sub-iterations $p$ ):

- construction of the diffusion matrix (see section (3.2)),

- initialization of $\delta u_{k, r}^{n+1, p=0}=\delta u_{k, r}^{n}$,

- calculation of $\boldsymbol{\beta}_{r}^{n+1, p}$ with (18) and (20),

- construction of the system on $e$ (25),

- calculation of $e_{j}^{n+1, p+1}$ by solving the linear system (25),

- deduction of $\boldsymbol{\gamma}_{r}^{n+1, p+1}$ with (18) and (20),

- deduction of $\boldsymbol{\delta} \boldsymbol{u}_{r}^{n+1, p+1}$ with (19),

- While (stopping criterion) $\max _{j}\left|e_{j}^{n+1, p+1}-e_{j}^{n+1, p}\right|>\epsilon$ and $\max _{k, r}\left|\delta u_{k, r}^{n+1, p+1}-\delta u_{k, r}^{n+1, p}\right|>$ $\epsilon$, with $\epsilon=10^{-9}$.

It is important to notice that there is only one loop on $p$ for the fixed point algorithm for $e$ and the implicit solve of $\boldsymbol{\delta} \boldsymbol{u}$.

\section{Numerical experiments}

In this last section, we are first interested in four $1 \mathrm{D}$ tests, which aim to validate the numerical scheme. We perform them on a Cartesian mesh and on an unstructured mesh, and compare the results with those of a Discontinuous Finite Element (DFE) code on a Cartesian mesh, and to an analytical solution if it exists. In order to test the scheme efficiency on a fully 2D configuration, we perform a fifth and final test: the lattice problem. This problem has been studied in numerous publications in the past decades [16, 28, 42, 43, 58,62, 66, 67], and the reader is invited to refer to them for comparison. Moreover, we also provide the results obtained with the DFE method.

Before detailing the test problems and the results, we give a short description of the DFE method we use for the sake of comparison. 


\subsection{Short description of the Discontinuous Finite Element (DFE) method}

325 port equation especially for photons. Its ability to give good results in transparent and opaque media and in the last case, without meshing at the mean free path length, explains this popularity. Several extensions to general polygonal (resp. polyhedral) meshes in 2D (resp. 3D) which degenerate in 1D into P1 DFE and still possess the of the radiative intensity at the vertices of the cell. The main features of the DFE method are:

- Accuracy The P1 DFE is second order accurate in space, at least on Cartesian mesh. As a consequence, positivity is not guaranteed if no limiter is used.

- Diffusion limit 1D P1 DFE method has the diffusion limit [51]. The limit scheme is a valid discretization of the diffusion equation by P1 continuous finite elements. 1D P1 DFE also captures the boundary layers accurately even using a crude mesh [51]. Note that the P0 Discontinuous Finite Element (the well known donor cell scheme), does not recover the diffusion limit.

- Implicitness In order not to constraint the time step, the scheme is made implicit in time. The source term which couples all directions is usually lagged. This allows to solve the angular discretized problem by direction. This technique is named the source iteration method. In opaque media, the convergence of the iterated process can be arbitrary slow. Solving between iterations some low order problem (DSA [4], TSA [63]) is often used to accelerate the convergence.

The discretization of the transport term leads to solve by direction a large linear system whose the unknowns are the values of the radiative intensity at the vertices of the cells. The properties of the transport operator allow to make this system block triangular by ordering the cells. Solving the whole linear system directly block by block is much cheaper than solving it with an iterative method. By cell, each block is a $N(j) \times N(j)$ matrix with $N(j)$ the number of the vertices of the cell. Finally, compute the unknowns needs to solve a $N(j) \times N(j)$ linear system per cell. Such ordering exists in 2D if only

convex cells are present. In 3D, this is no longer the case. Graph algorithms allow to recover such an ordering at the expense of lagging some intensities at the previous iteration in the source iteration method [61]. 
In this article, the Bailey method for the spatial discretization and an acceleration technique TSA [63]) is used.

\section{2. $1 D$ tests}

Regarding the first four tests:

- The first test free streaming limit aims to check the consistency and the good behavior (stability, precision) of the new scheme within the free streaming limit.

- The second test diffusion in homogeneous media aims to check the AsymptoticPreserving property of the new scheme, as well as its ability to support arbitrarily large time steps as would do a classical solver for implicit diffusion.

- The third test transport/diffusion interfaces aims to evaluate the behavior (robustness, precision) of the new scheme in the presence of an heterogeneous media, made of a succession of transport zones and diffusion zones.

- The fourth test stationary boundary layer aims to check the ability of the new scheme to solve a non-trivial stationary problem, and to evaluate the accuracy of the results on a coarse mesh in the presence of a boundary layer.

In the figures, one can observe two or three curves: one curve corresponding to our finite volume scheme (named FV), one curve corresponding to the DFE method, and for the tests one and two, a last curve corresponds to the analytical solution. To compare Cartesian mesh to unstructured mesh results in $1 \mathrm{D}$, we use the spatial discretization of the boundary obtained on the Cartesian mesh as the basis for the unstructured discretization. For instance, when we say that the discretization corresponds to 100 cells along the $x$-axis, it means that if we use 100 edges of squares (resp. 100 edges of triangles) discretizing the $y=y_{\min }$ boundary for the Cartesian mesh (resp. for the unstructured mesh).

\subsubsection{First test: free streaming limit}

The analytical solution is obtained using the characteristic method. We work on the domain $\Omega=[-1,+1] \times[-0.08,0.08]$. The type of unstructured mesh we use for this test is shown on Fig. 5. Initial condition is

$$
u(t=0, \boldsymbol{x}, \mu)=\mathbb{1}_{x \in[-0.5 ;+0.5]}, \quad q=\sigma_{a}=\sigma_{s}=0 .
$$

Zero incoming flux conditions are applied on boundaries

$$
u\left(t, x=-1, y, \boldsymbol{\omega} \cdot \boldsymbol{n}_{\boldsymbol{x}}>0\right)=u\left(t, x=1, y, \boldsymbol{\omega} \cdot \boldsymbol{n}_{\boldsymbol{x}}<0\right)=0,
$$


mesh test 1

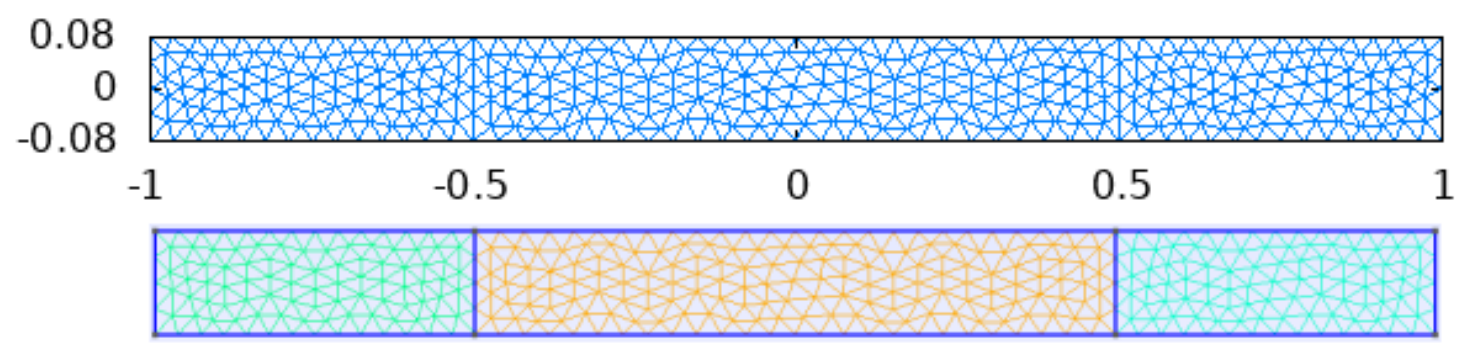

Figure 5: Unstructured mesh used for the free streaming problem.

where $\boldsymbol{n}_{\boldsymbol{x}}=(1,0)^{T}$ is the unit normal along the $x$ axis. Final time of the calculation is $t=0.2$, and we use a time-step $\Delta t=0.02$. The number of cells along the $x$-axis is 100 for the Cartesian cells. We compare the plot $e(\boldsymbol{x})$ obtained with our new method (FV) to the analytical solution, and the DFE result, for $N=1$ ( $K=4$ directions, Fig. 6 and 7), and $N=6(K=144$ directions Fig. 8 and 9$)$.

Results are very close to each other and in good agreement with the analytical solution, whatever the kind of mesh (Cartesian or unstructured). Our scheme is more diffusive than DFE, but DFE exhibits small overshoots and undershoots, which makes the solution goes below zero, which relies on the second-order accuracy of these methods.

The angular convergence is illustrated for $N=6(K=144)$, on Fig. 8 and 9 .

We have done tests with very large CFL numbers, to verify that the new method indeed restores the purely transport regime in an unconditionally stable way, whereas the algorithmic complexity of the solver is comparable to the one of an implicit diffusion type solver.

\subsubsection{Second test: diffusion in homogeneous media}

In this test, initial and boundary conditions are unchanged, except for the scattering cross section, which is taken as $\sigma_{s}=10^{5}$. In these conditions, the problem is steep enough for the diffusion limit to be valid. This problem is intended to verify that our new scheme is Asymptotic Preserving. In addition, we do not expect any dependence of the result w.r.t. angular discretization. The analytical solution of the test is the solution of the diffusion equation $\partial_{t} e=D \partial_{x^{2}}^{2} e$ with $D=v /\left(3 \sigma_{s}\right)$ associated with the initial condition $e(t=0, x)=\mathbb{1}_{x \in[-0,5 ;+0,5]}$.

The time-step $\Delta t=1$ is used for all the results in this section. The type of unstructured mesh used for this test is the same than for the previous one (see Fig. 5, with 


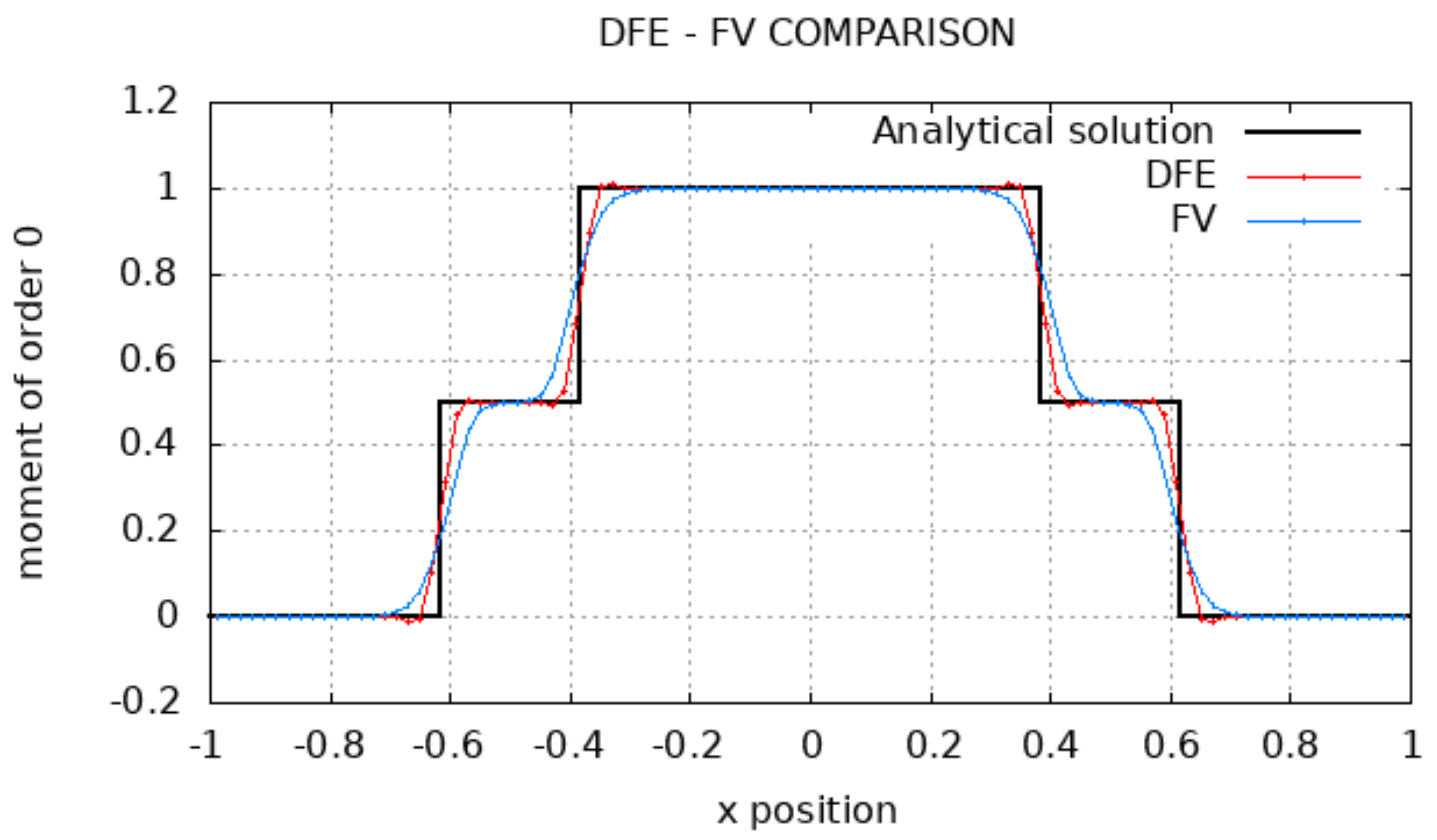

Figure 6: Comparison of $e$ between finite volume and DFE, on Cartesian mesh, for the free streaming problem, with $N=1(K=4)$ and $\Delta t=0.02$.

100 cells along the $x$-axis. We plot the curve of $e(\boldsymbol{x})$ at time $t=10^{3}$, with two different angular discretizations $N=1(K=4)$, see Fig. 10 and 11 and $N=6(K=144)$, see Fig. 12 and 13. We observe a very good agreement between the two methods. The solution does not depend on the angular discretization, and is superimposed to the analytical solution, even on these coarse meshes. The unstructured mesh result is as satisfactory as that of the Cartesian mesh, and respects the maximum principle on $e$. Moreover, it behaves like a classical implicit diffusion solver, whereas the DFE method requires the use of an acceleration technique (DSA or TSA).

\subsubsection{Third test: transport/diffusion interfaces}

This problem requires a sufficient number of angular directions to be interesting, and we take $N=11$ ( $K=484$ directions) for the finite volume method and $K=496$ 


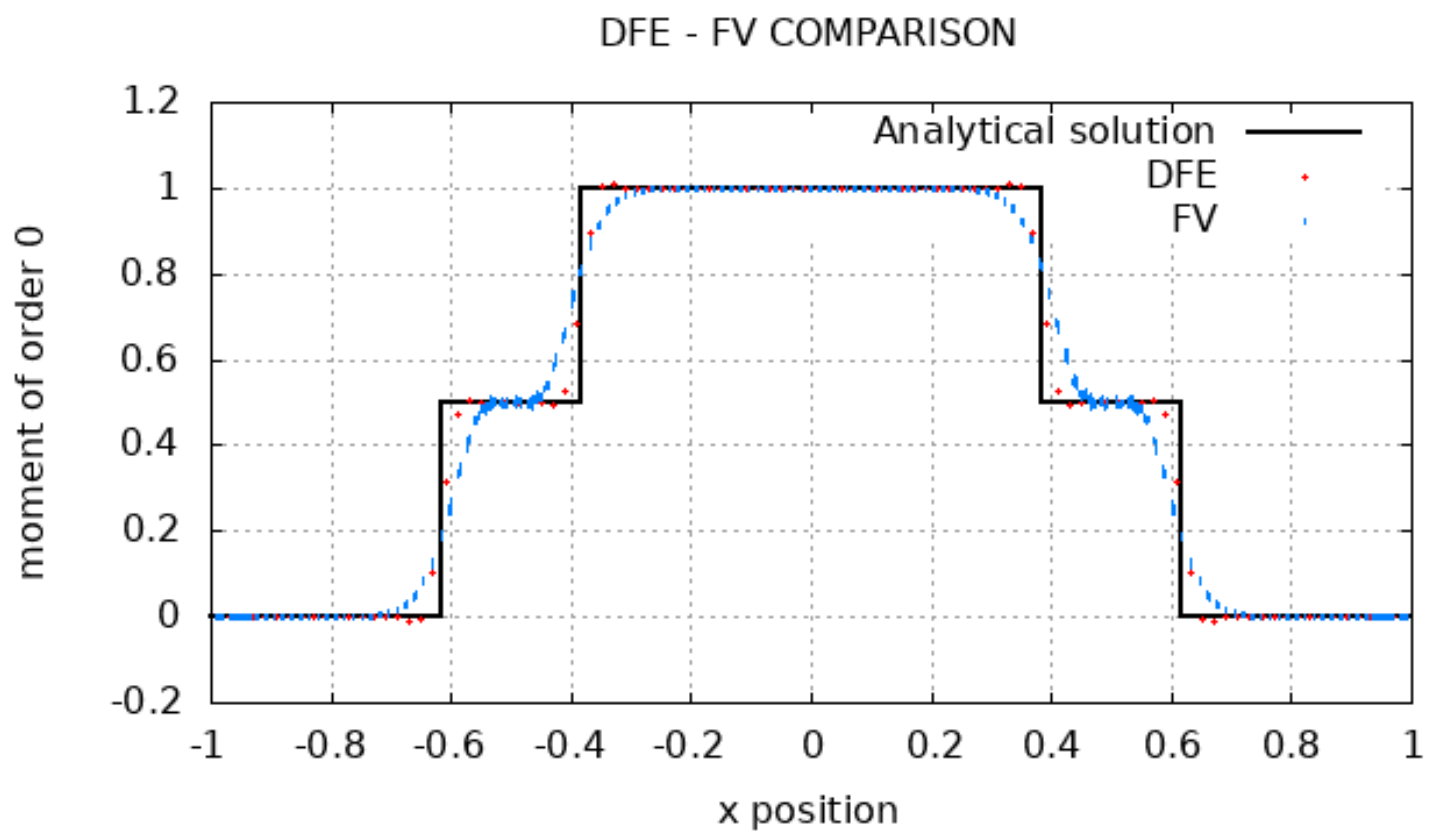

Figure 7: Comparison of $e$ between finite volume and DFE, on unstructured mesh, for the free streaming problem, with $N=1(K=4)$ and $\Delta t=0.02$.

directions for the in the DFE method in the following ${ }^{1}$. For this test, the scattering cross section is discontinuous:

$$
\sigma_{s}=10^{2} \mathbb{1}_{|x| \in[0.3 ; 0.4]}+10^{3} \mathbb{1}_{|x| \in[0.5 ; 0.6]}+10^{4} \mathbb{1}_{|x| \in[0.7 ; 0.8]} \mathbb{1}_{|x| \geq 0.9},
$$

and we have

$$
q=0, \quad \sigma_{a}=0
$$

\footnotetext{
${ }^{1}$ The angular quadrature formula implemented being different for the two methods, we cannot use exactly the same number of directions. However, we checked that the angular convergence is achieved.
} 


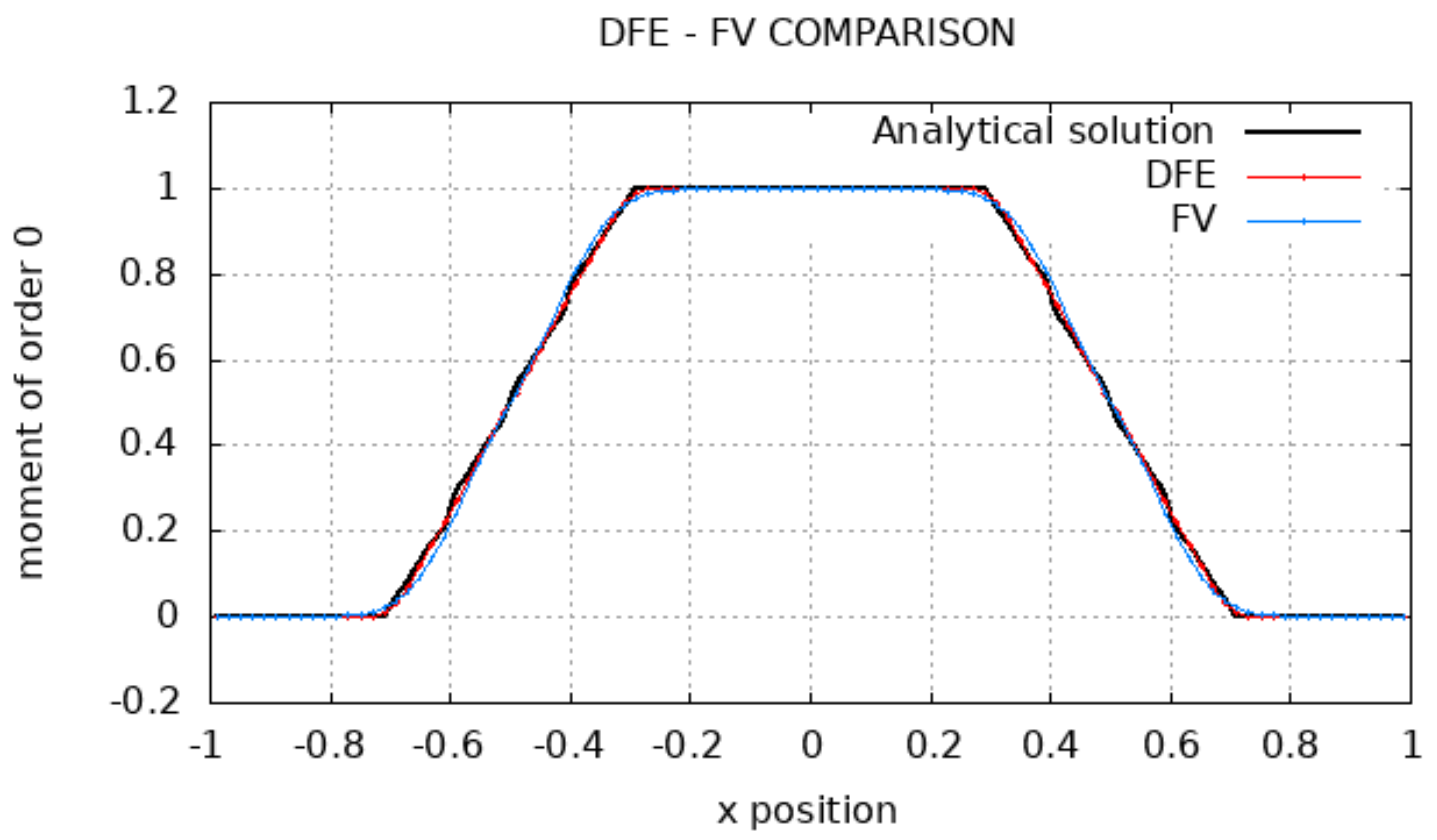

Figure 8: Comparison of the angular moment $e$ between finite volume and DFE, on Cartesian mesh, for the free streaming problem, with $N=12(K=576$ for FV and $K=544$ for DFE) and $\Delta t=0.02$.

The initial condition is

$$
u(t=0, \boldsymbol{x}, \mu)=\mathbb{1}_{x \in[-0.1 ;+0.1]} .
$$

The unstructured mesh is depicted on Fig. 14.

We draw the profile $e(t, x)$ at time $t=1,10$ and 100, and compare our new scheme with the DFE method. Results for $\mathbf{t}=\mathbf{1}$ are given on Fig. 15 and 16.

All the curves agree well for $|x|>0,4$. The precision with which these waves are captured in the $x<0.3$ zone obviously does not influence the precision for $x \geq 0.3$, which is reassuring. In particular, the height of the flat area for $x \in] 0.4 ; 0.5[$ fits perfectly between methods, whatever the mesh (Cartesian or unstructured).

Results for $\mathbf{t}=\mathbf{1 0}$ and for $\mathbf{t}=\mathbf{1 0 0}$ are displayed on Fig. 17 and 18 and Fig. 19 and 20, respectively. The results of our method fit very well the results of the DFE 


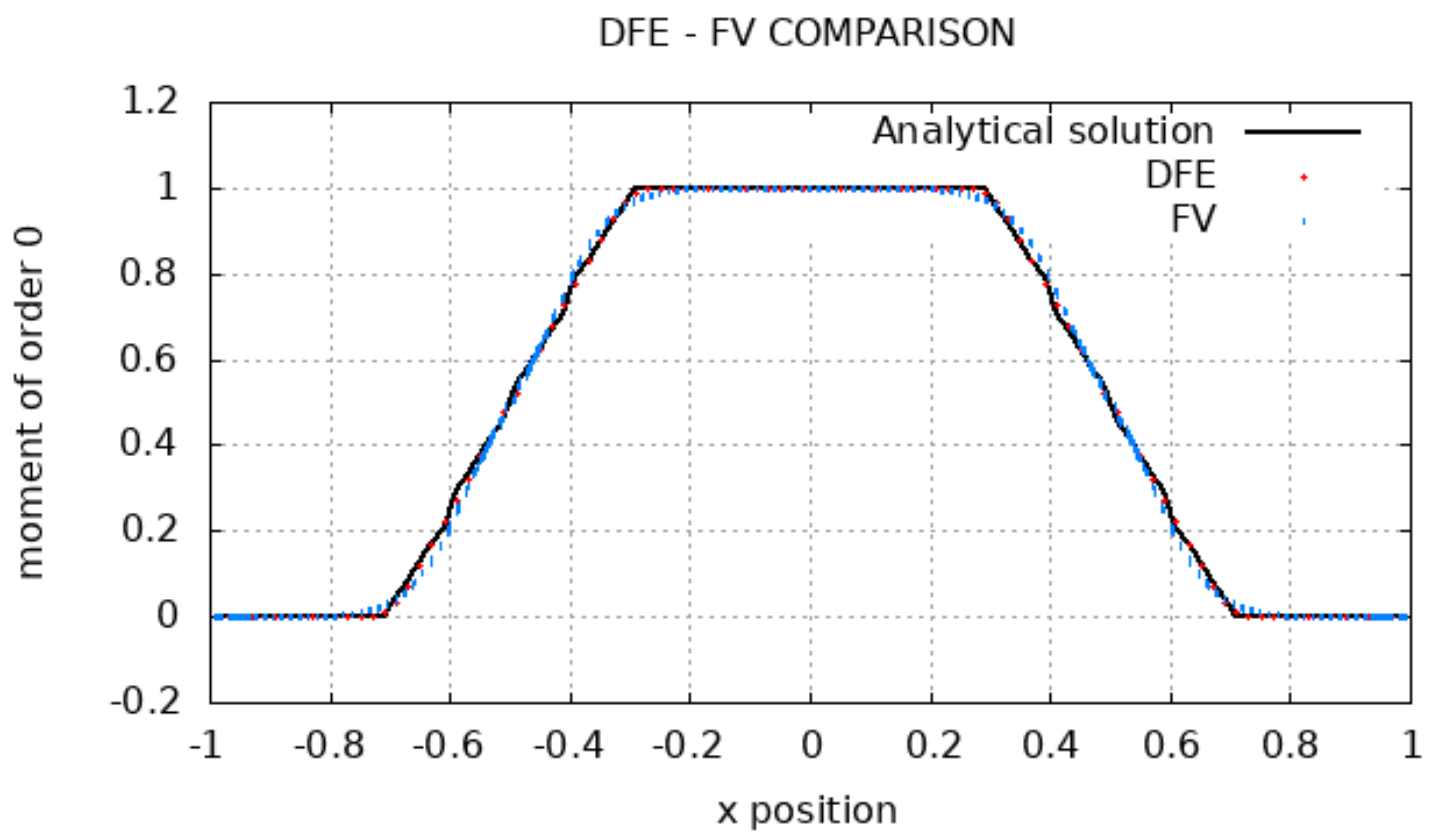

Figure 9: Comparison of the angular moment $e$ between finite volume and DFE, on unstructured mesh, for the free streaming problem, with $N=12(K=576$ for FV and $K=544$ for DFE) and $\Delta t=0.02$.

method. In addition, the positions of the discontinuities of the derivative of $e$ match those of the cross section, as expected. We observe that our method fulfills the maximum principle on $e$, while small oscillations occur in the DFE calculations, again because of the second order accuracy of the method.

\subsubsection{Fourth test: stationary boundary layer}

This test is freely inspired from the analysis of Larsen \& Morel [51]. It aims at verifying the ability of our method to handle unresolved boundary layers due to the steep variation of the cross sections. The objective is to be able to calculate the correct slope of $e$ on coarse meshes. As for the previous problem, this problem requires a sufficient number of angular directions to be interesting. We take $N=4$ ( $K=64$ directions in 2D) in the following. We are then interested in the profile 


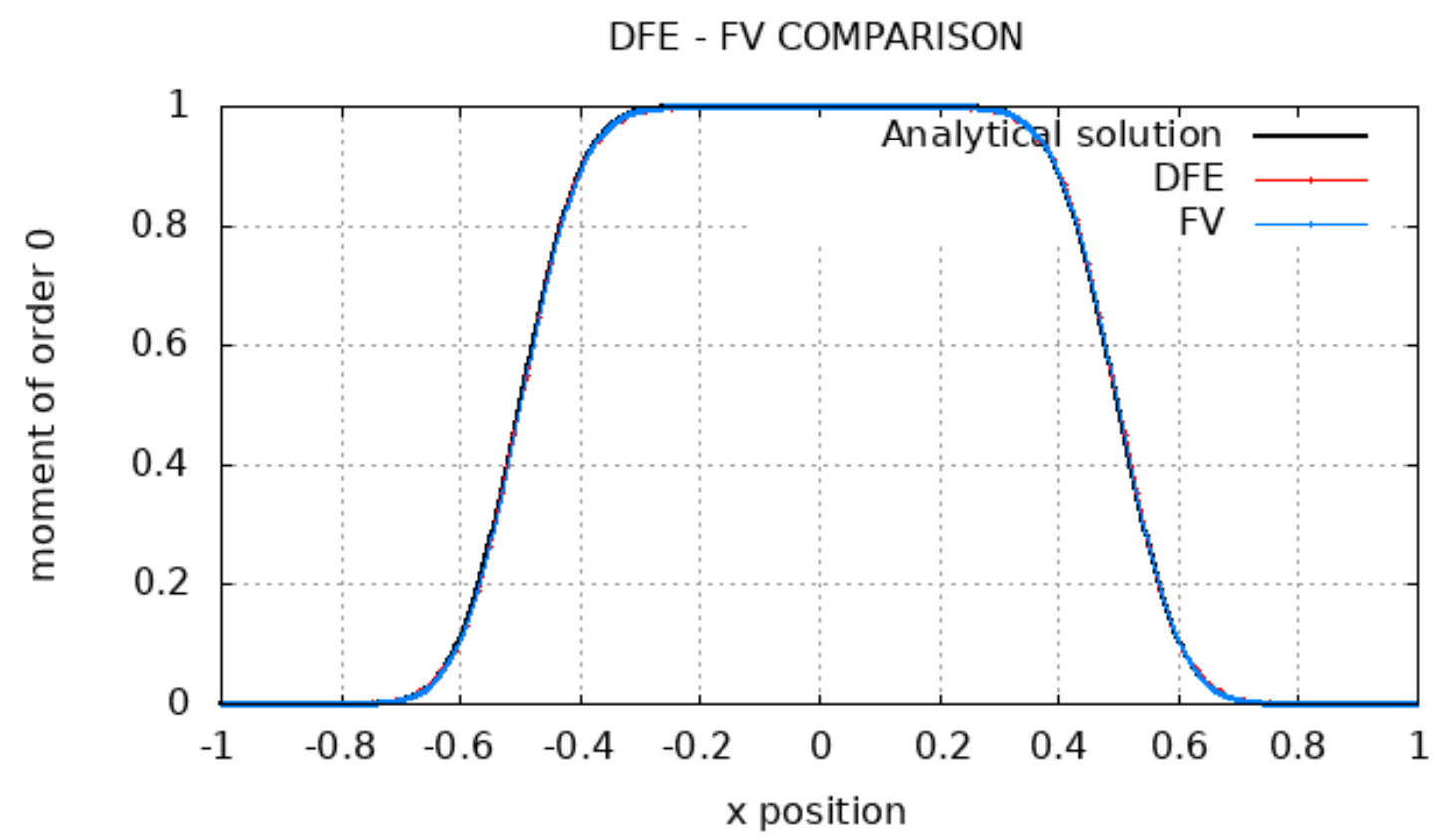

Figure 10: Comparison of the angular moment $e$ between finite volume and DFE, on Cartesian mesh, for the diffusion in homogeneous media problem, with $N=1(K=4)$ and $\Delta t=10$.

of the moment of order $0, e$, in particular close to the interface located at $x=0.8$ where a boundary layer develops.

For this test, we have :

$$
q=\mathbb{1}_{|x| \leq 0,1}, \sigma_{a}=\mathbb{1}_{|x|<0.8}+10 \mathbb{1}_{|x| \in[1.0 ; 1.2]} \text { and } \sigma_{s}=10^{2} \mathbb{1}_{|x| \in[0.8 ; 1.0]} .
$$

The initial condition is :

$$
u(t=0, \boldsymbol{x}, \mu)=0 .
$$

The code used being unsteady, we reach the solution of this stationary problem by taking in practice:

$$
v=1, \quad u(t=0, \boldsymbol{x}, \mu)=0, \quad t=10^{9},
$$




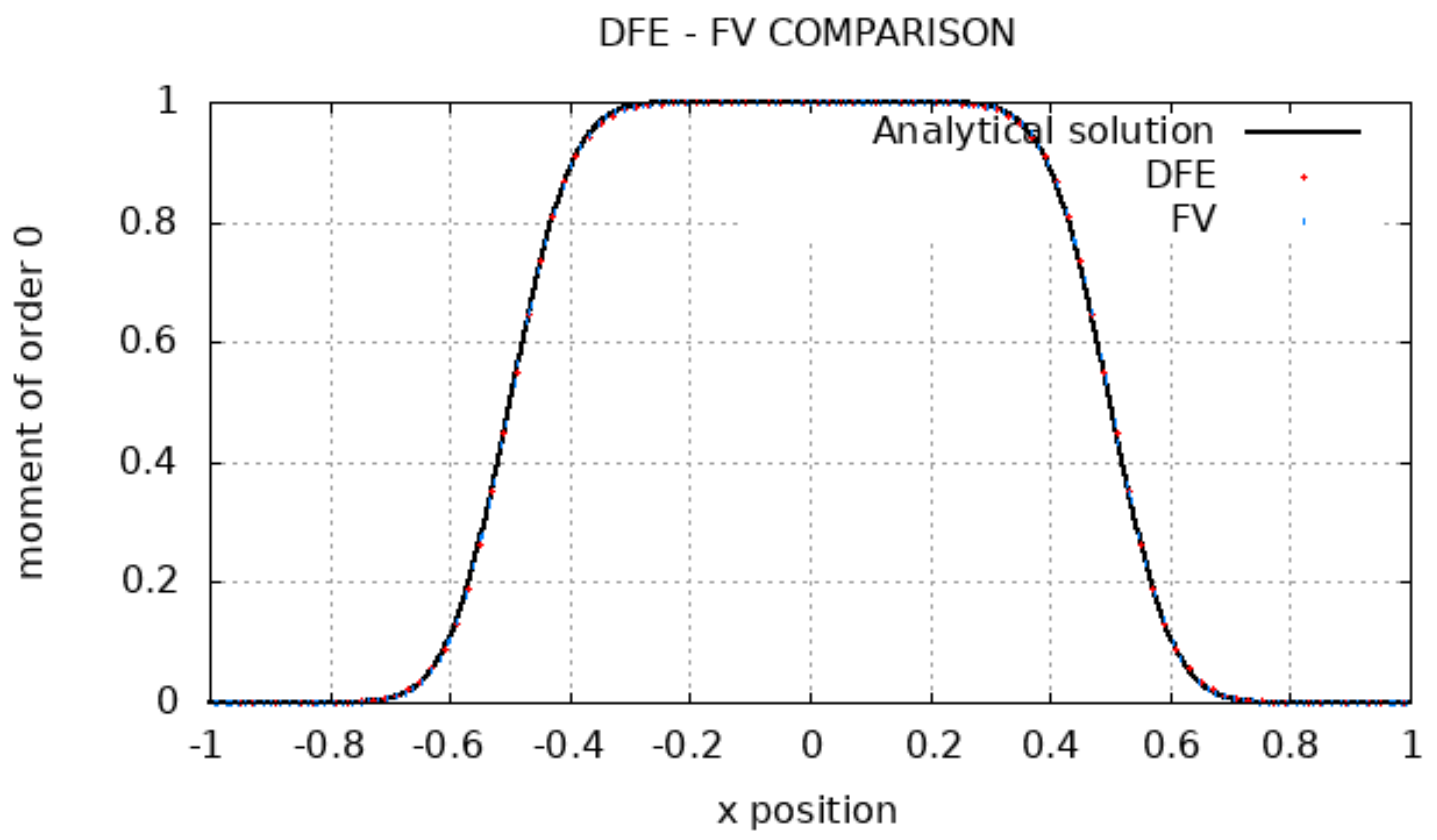

Figure 11: Comparison of the angular moment $e$ between finite volume and DFE, on unstructured mesh, for the diffusion in homogeneous media problem, with $N=1(K=4), \Delta t=10$.

with a single iteration in time $\left(\Delta t=10^{9}\right)$. The presence of an absorption term $\sigma_{a}>0$ quickly makes the solution tends towards 0 for $|x|>1$ and thus preserves it from the influence of the boundaries of the domain.

We take $\Omega=[-1.2,+1.2] \times[-1 ;+1]$ for the Cartesian mesh. On unstructured mesh, we work on the domain $\Omega=[-1.2,+1.2] \times[-0.2,0.2]$. An example of unstructured mesh used for this test is shown on Fig. 21.

In order to simplify the initialization (of $\delta u$ and $e$ ), we divide the calculation domain in three different areas.

The results on Cartesian and unstructured meshes are displayed on Fig. 22. For the comparison purpose, the DFE method is used with a very fine mesh, to obtain a DFE reference solution. Our results, on both structured and unstructured grids, agree very well with the reference DFE calculation. 


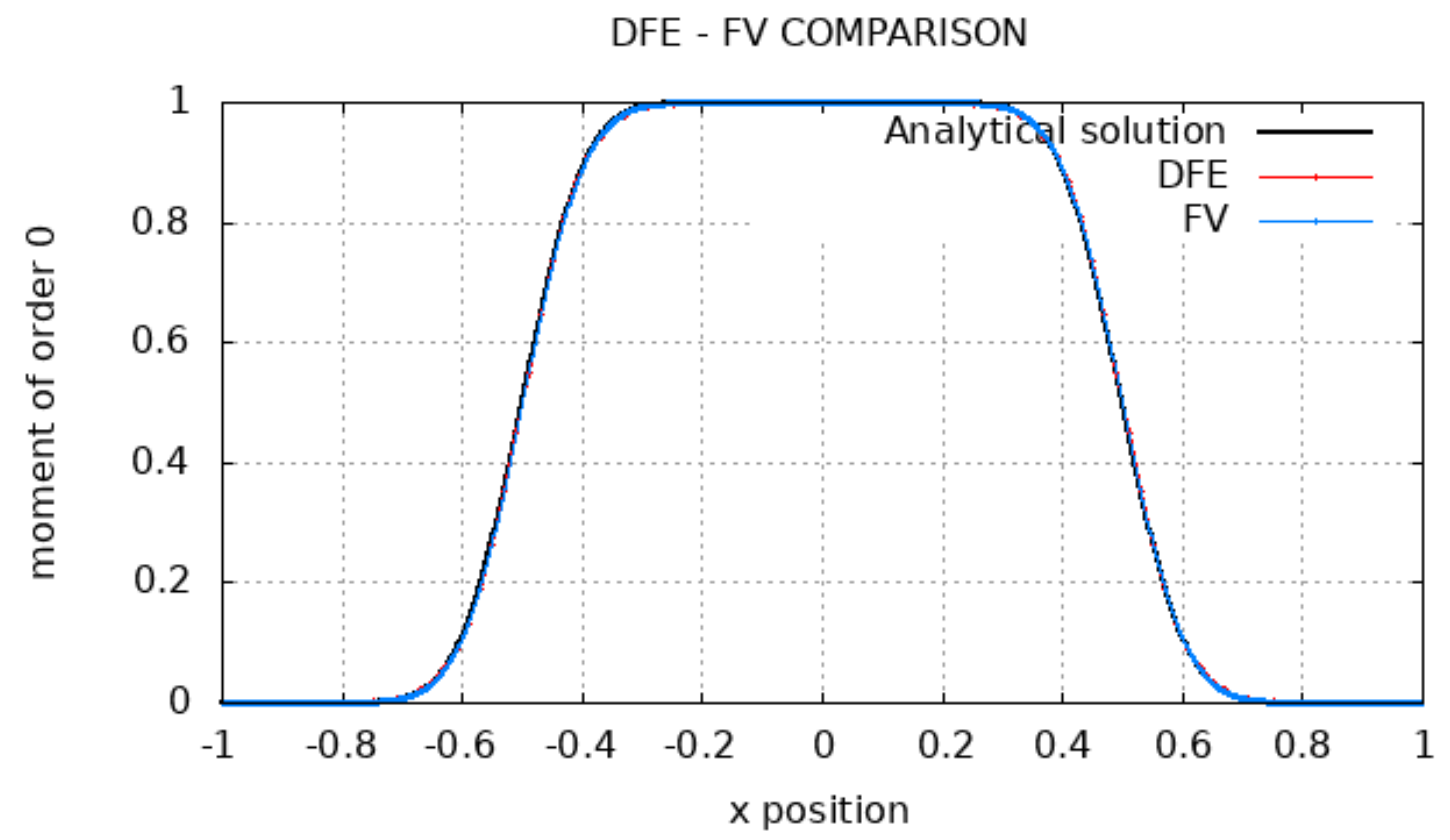

Figure 12: Comparison of the angular moment $e$ between finite volume and DFE, on Cartesian mesh, for the diffusion in homogeneous media problem, with $\Delta t=10$.

We now focus on the behaviour of $e$ at the boundary layer, close to $x=0.8$. Results are given on Fig. 24.

On this coarse mesh, the discontinuity of $e$ at $x=0.8$ is poorly restored in the $\mathrm{FV}$ code, but the good agreement of the slope in the diffusion zone $(x>0.8)$ testifies that the net flux at the interface is correct. With the DFE code, we can observe a discontinuity of $e$ at $x=0.8$. As DFE methods are known to very well capture these boundary layers [51], the very good agreement with our method is very satisfactory. It means that the net flux at the interface is correctly calculated by the new method.

Then we perform a spatial convergence study on the test 4 . Results are displayed on Fig. 25. It shows that the slope is correctly calculated even on the 24 cells mesh. 


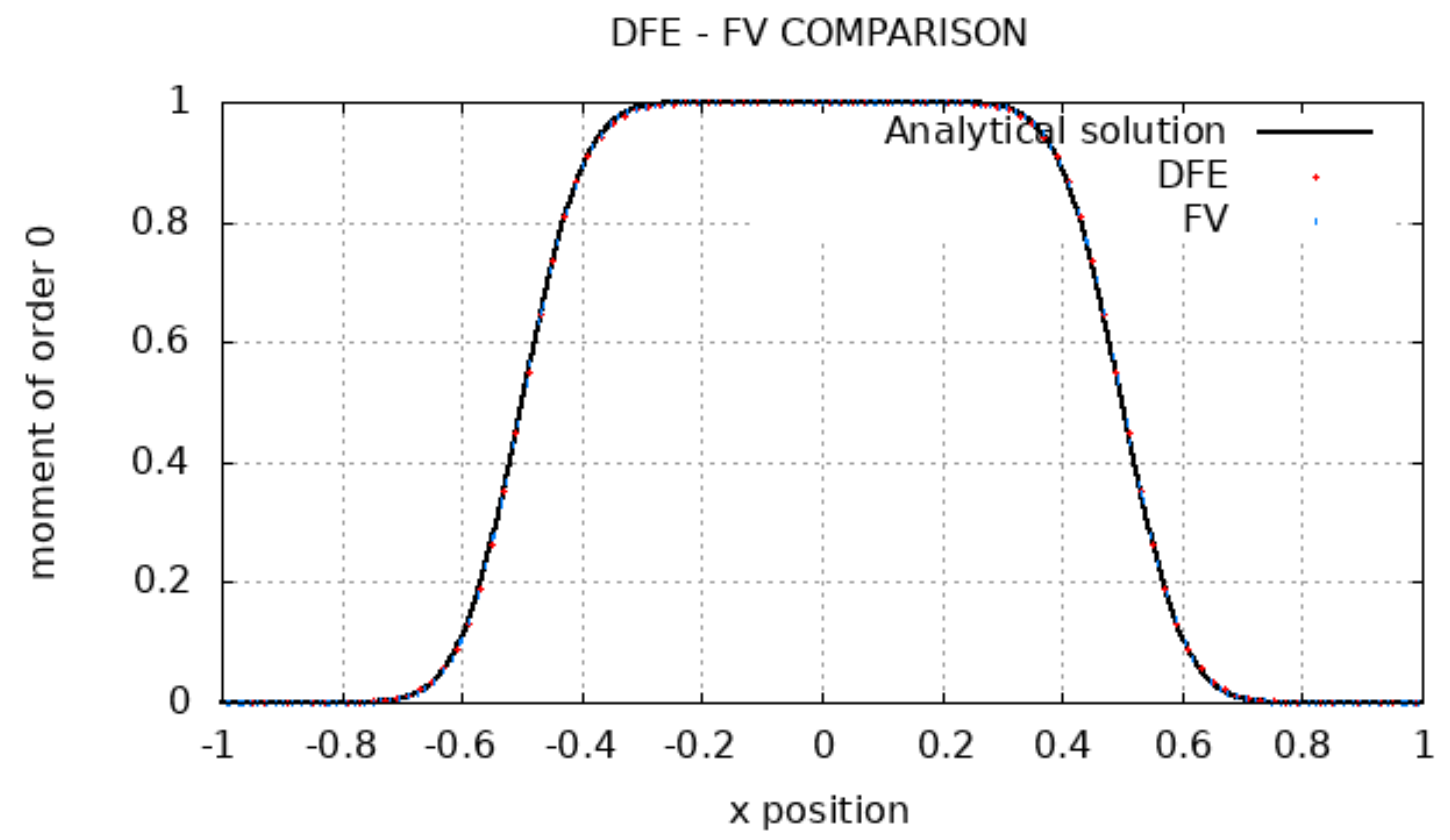

Figure 13: Comparison of the angular moment $e$ between finite volume and DFE, on unstructured mesh, for the diffusion in homogeneous media problem, with $\Delta t=10$.

In addition, it shows the quick convergence of our new algorithm to the reference DFE solution obtained on a very fine mesh (20000 degrees of freedom).

\subsection{Lattice problem}

We consider the lattice problem as described in [15]. The domain is $\Omega=[0,7] \times$ $[0,7]$.

We divide the domain as in Fig. 26. The white area, bulk of the lattice, corresponds to a scattering material and eleven blue area to absorbing regions. The black region in the center is a scattering region, where a source of photons is emitted. Let $\Omega_{A}$ be the union of the eleven striped squares (absorption region) and let $\Omega_{C}=[3,4]^{2}$ 
mesh test 3

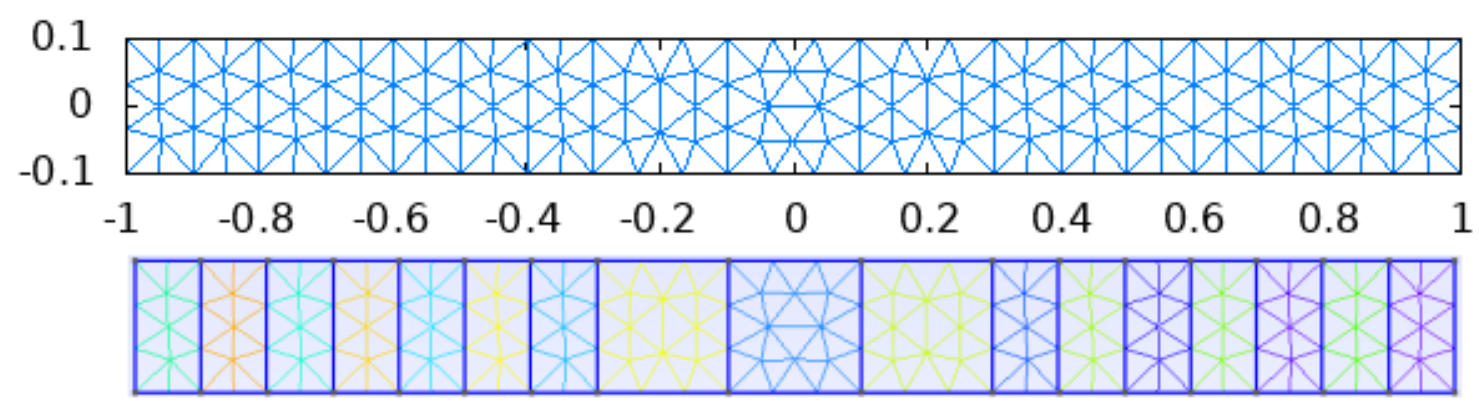

Figure 14: Unstructured mesh used for the transport/diffusion interfaces problem.

be the center region.

$$
\text { Then, we have : }\left\{\begin{array}{l}
\sigma_{a}(\boldsymbol{x})=10 \text { and } \sigma_{s}(\boldsymbol{x})=0 \text { if } \boldsymbol{x} \in \Omega_{A} \\
\sigma_{a}(\boldsymbol{x})=0 \text { and } \sigma_{s}(\boldsymbol{x})=1 \text { else }
\end{array}\right.
$$

The source is concentrated in the center (in the black square), such as

$$
\begin{cases}s(\boldsymbol{x})=1 & \text { if } \boldsymbol{x} \in \Omega_{C} \\ s(\boldsymbol{x})=0 & \text { else }\end{cases}
$$

We used a $140 \times 140$ mesh, with $\Delta t=10^{-2}$. The test is performed on Cartesian and unstructured meshes for our method, and on a Cartesian mesh for the DFE method. The kind of unstructured mesh used for this first test is given on Fig. 27.

We plot the map of $e(\boldsymbol{x}, t)$ at time $t=3.2$ on Fig. 28 to Fig. 33. Our results are comparable to those obtained in other publications [16, 28, 42, 43, 58, 62, 66, 67].

We compare our results in 2D on Cartesian mesh, unstructured mesh and a test carried out with DFE on Fig. 34 and Fig. 35. The left figure represents the results obtained on Cartesian mesh with the new scheme, the right figure represents the results obtained with the new scheme on unstructured mesh, and finally, the middle figure is that obtained with the DFE. 


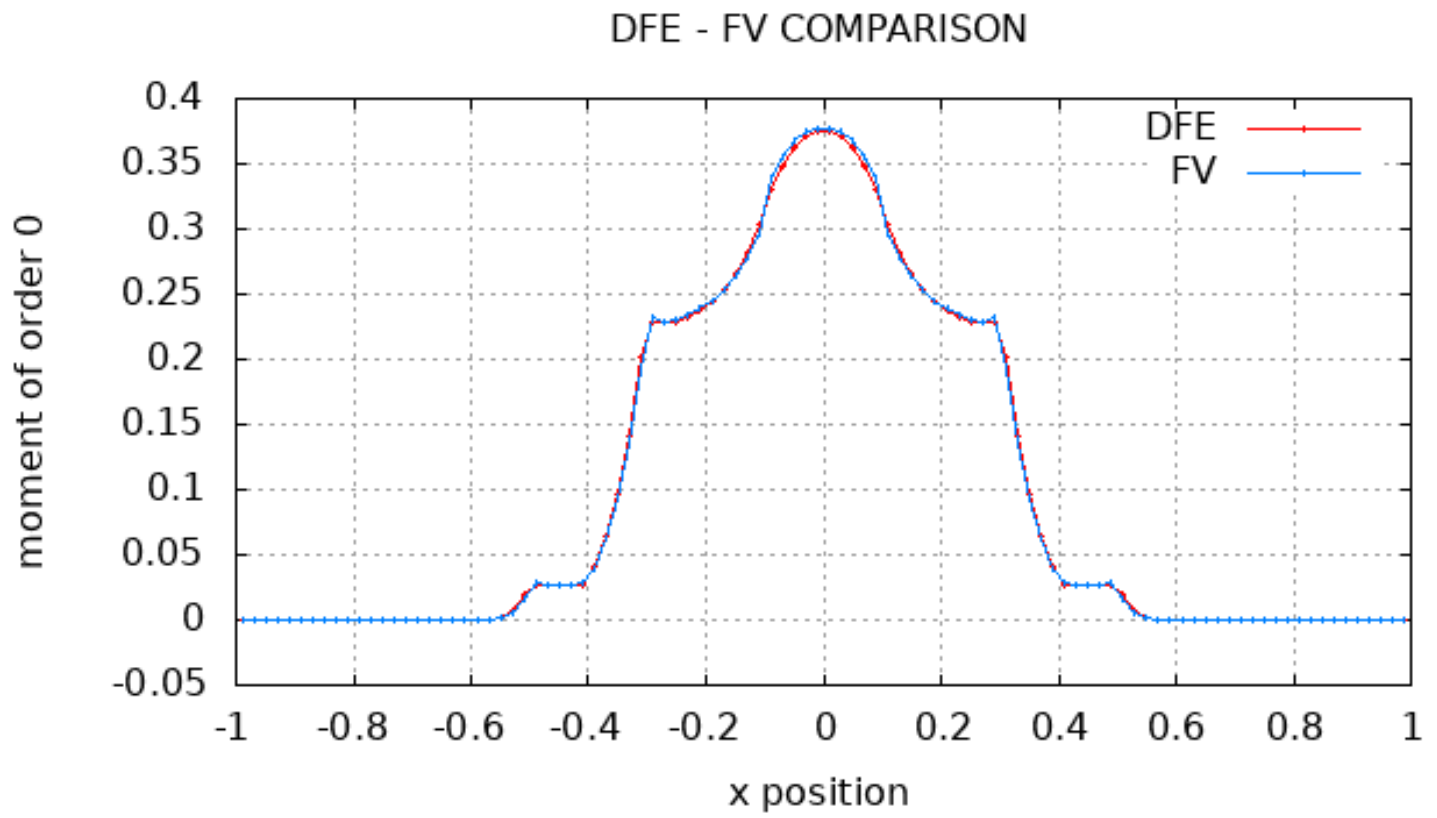

Figure 15: Comparison of the angular moment $e$ between finite volume and DFE, on Cartesian mesh, for the transport/diffusion interfaces problem, with $N=11(K=484)$ and $\Delta t=1$, plot at time $t=1$.

\section{Conclusion}

We have presented in this article a finite-volume scheme for a linear transport equation derived from a linearization of the radiative transfer equations. This scheme as been implemented on 2D unstructured meshes, and satisfies the following properties:

$\mathbf{P 1}$ to be consistent on general meshes,

P2 to enforce the conservation of radiative energy,

P3 to be able to handle all the radiation regimes from free-streaming to diffusion (Asymptotic Preserving), 


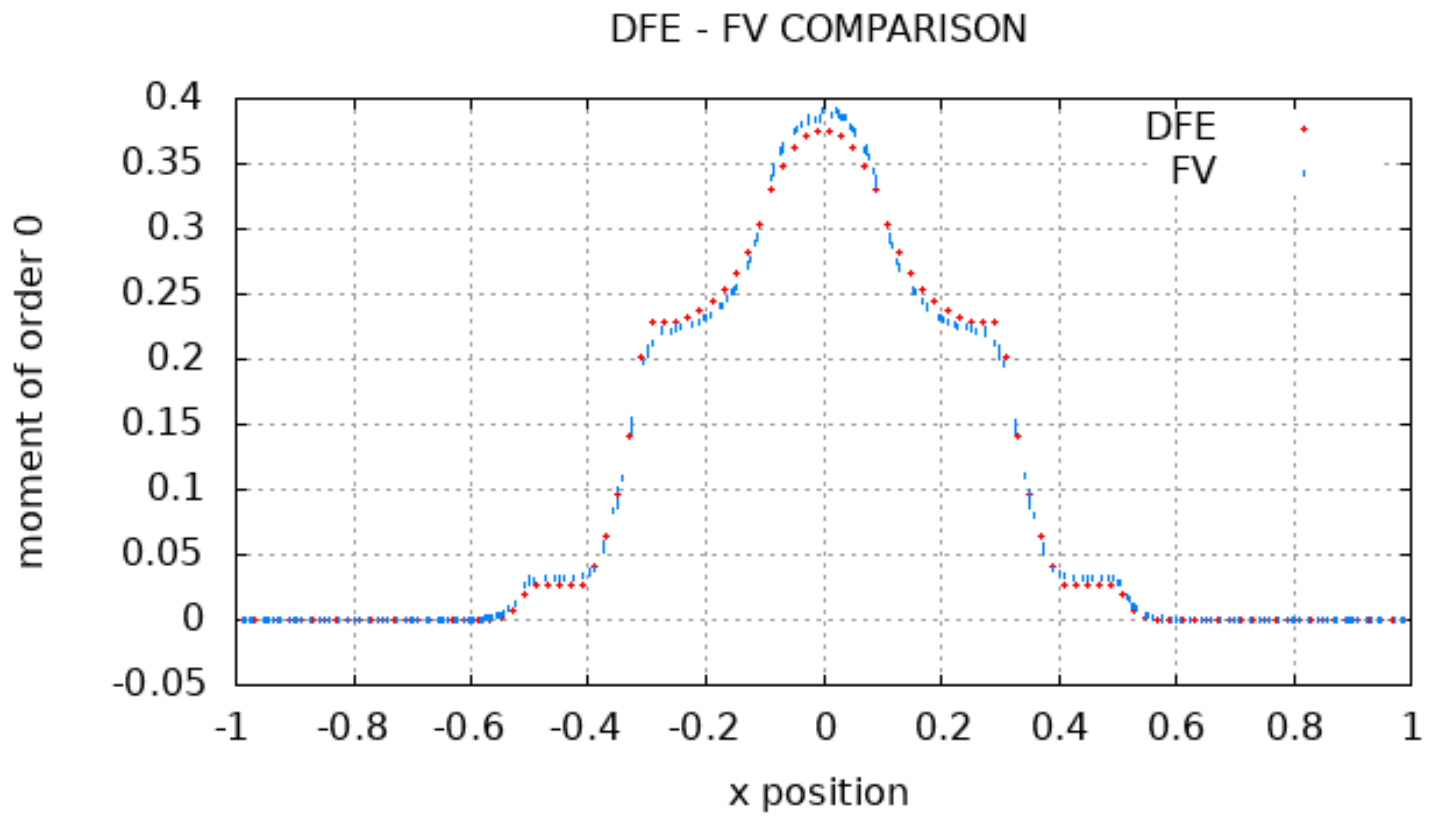

Figure 16: Comparison of the angular moment $e$ between finite volume and DFE, on unstructured mesh, for the transport/diffusion interfaces problem, with $N=11(K=484)$ and $\Delta t=1$, plot at time $t=1$.

$\mathbf{P} 4$ to have the radiative energy degrees of freedom located at the centers of the elements to ensure the compatibility with the hydrodynamic scheme.

P5 to allow us to use the limit diffusion scheme of our choice.

As our scheme is implicit, we used a system of sub-iterations to avoid to solve a global linear system (of size equal to the cells number $\times$ directions number), while remaining stable. In addition, this sub-iterations system is acting as a fixed point loop, in order to get rid of the non-linearity of the diffusion scheme. Thanks to the properties of our diffusion scheme, we enforce the energy to remain positive in this regime, for which radiation and matter temperature are strongly coupled. We have conducted numerical 1D tests on structured and unstructured 2D meshes, which assess that the 


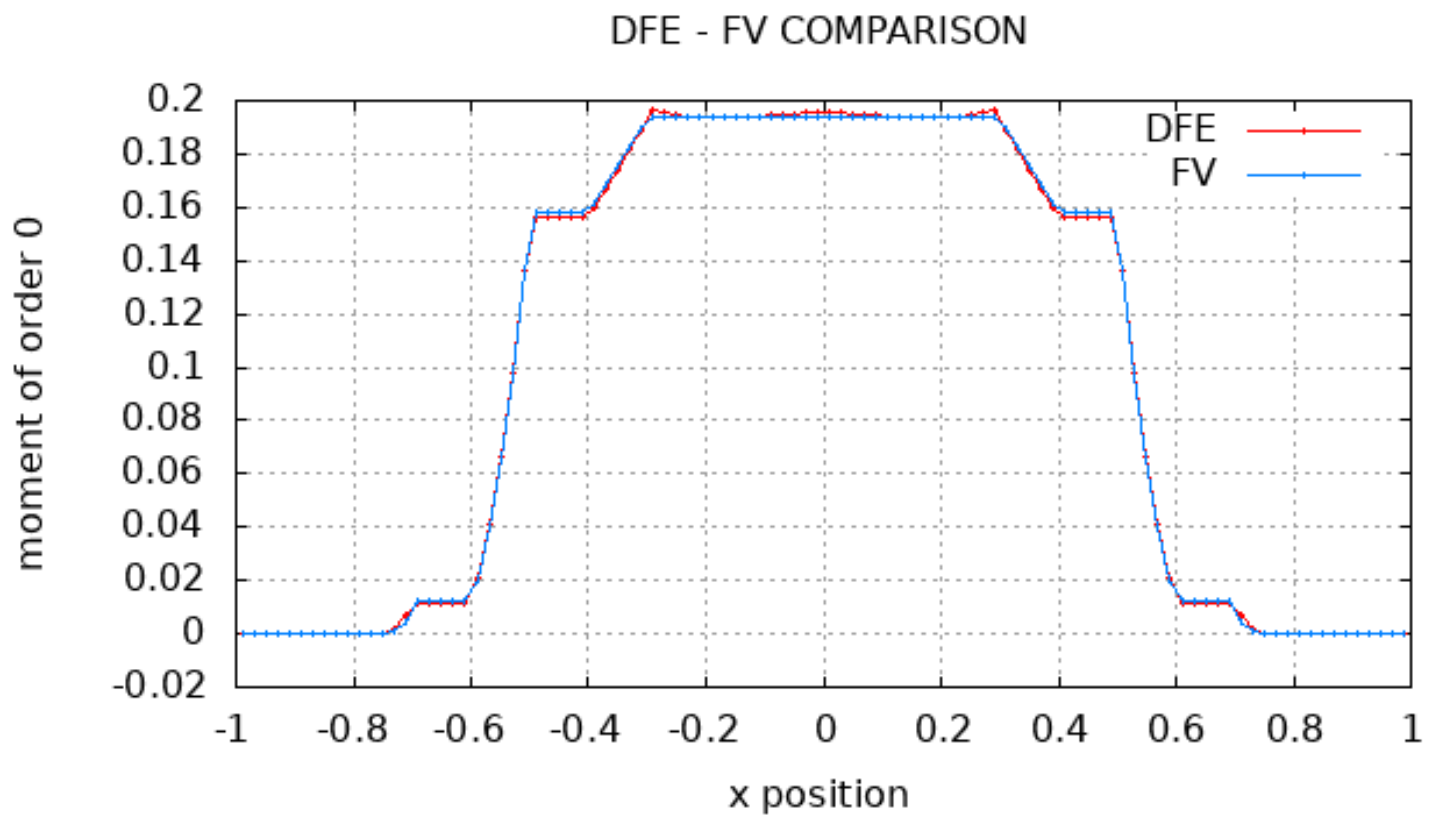

Figure 17: Comparison of the angular moment $e$ between finite volume and DFE, on Cartesian mesh, for the transport/diffusion interfaces problem, with $N=11(K=484)$ and $\Delta t=1$, plot at time $t=10$.

expected properties are respected. Finally, we carried out the Lattice problem test to compare our results with existing methods, in particular the Discontinuous Finite Element (DFE) discretization. In the future, we aim at designing a scheme which enforces the positivity of the energy in all regimes. 


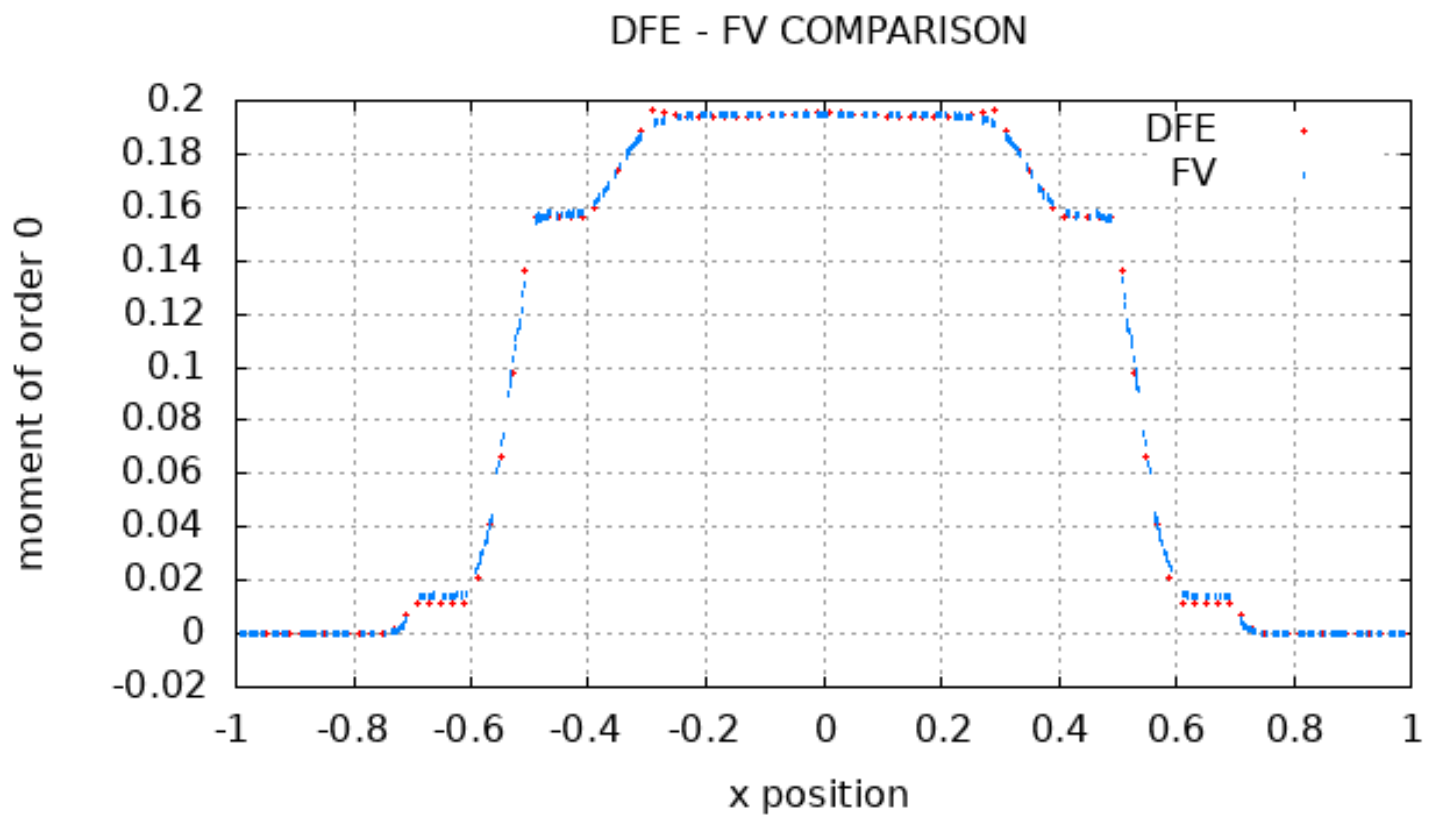

Figure 18: Comparison of the angular moment $e$ between finite volume and DFE, on unstructured mesh, for the transport/diffusion interfaces problem, with $N=11(K=484)$ and $\Delta t=1$, plot at time $t=10$.

\section{Appendix A. Proof of the accuracy of the boundary integral approxima- tion}

In the following we prove that

$$
\left\{\begin{array}{l}
\frac{1}{|\partial j|} \int_{\partial j} \varphi \boldsymbol{n}=\frac{1}{|\partial j|} \sum_{l(j)}|l| \varphi_{l} \mathbf{n}_{j l}+\mathcal{O}\left(h^{2}\right), \\
\frac{1}{|\partial j|} \int_{\partial j} \varphi \boldsymbol{n}=\frac{1}{|\partial j|} \sum_{r(j)} \varphi_{r} \mathbf{C}_{j r}+\mathcal{O}\left(h^{2}\right),
\end{array}\right.
$$




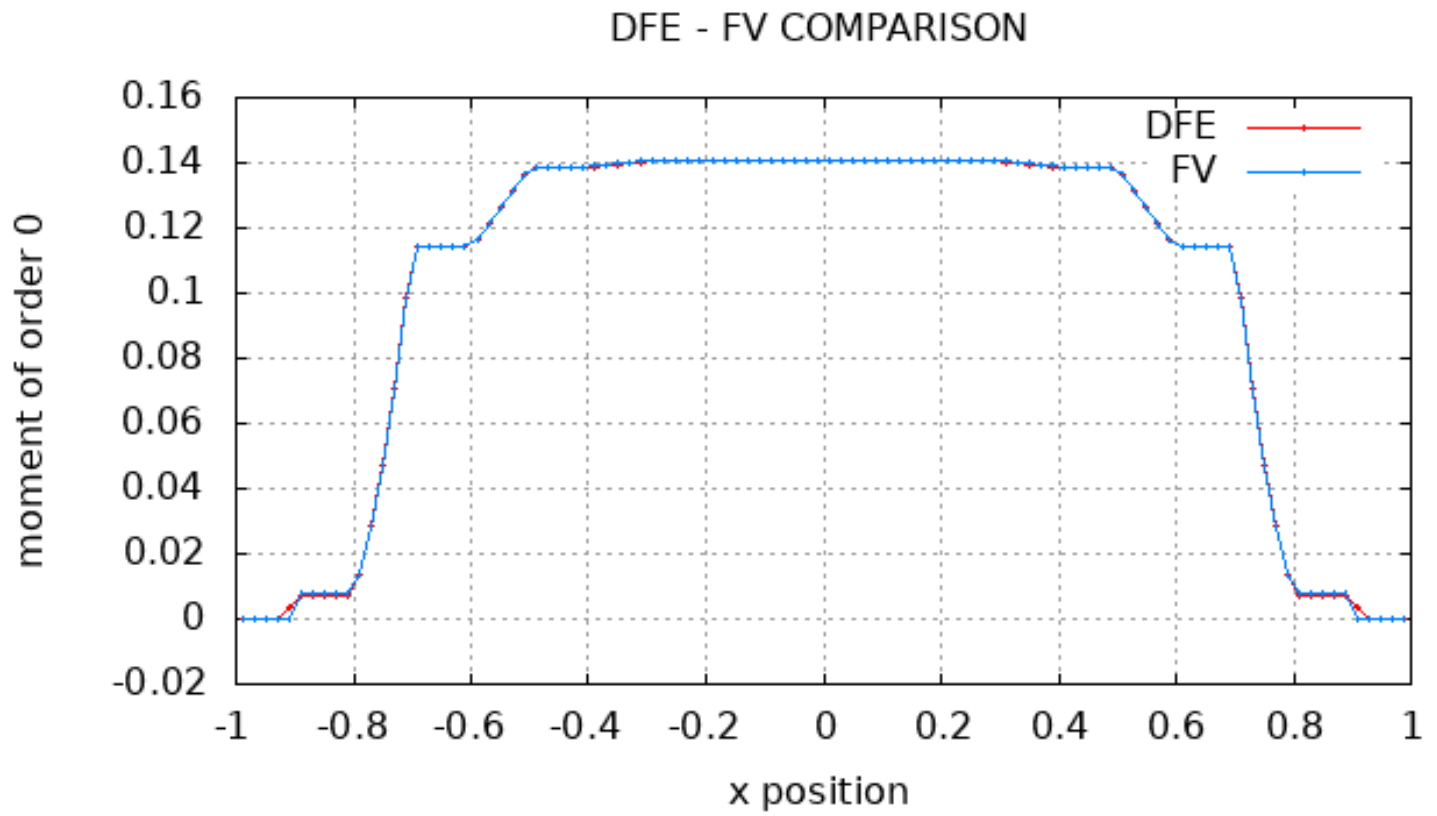

Figure 19: Comparison of the angular moment $e$ between finite volume and DFE, on Cartesian mesh, for the transport/diffusion interfaces problem, with $N=11(K=484)$ and $\Delta t=1$, plot at time $t=100$.

where $h=\max _{l(j)}|l|$.

$$
\begin{aligned}
& \int_{\partial j} \varphi \boldsymbol{n}=\sum_{l(j)} \int_{l} \varphi \boldsymbol{n}_{j l}, \quad \quad 1 \text { are straight } \\
& =\sum_{l(j)} \int_{l}\left(\varphi_{l} \boldsymbol{n}_{j l}+(\nabla \varphi)_{l}\left(\boldsymbol{x}-\boldsymbol{x}_{l}\right)+\mathcal{O}\left(h^{2}\right)\right), \text { Taylor expansion, } \\
& =\sum_{l(j)}|l| \varphi_{l} \boldsymbol{n}_{j l}+\mathcal{O}\left(h^{3}\right), \quad \quad \int_{l(j)}\left(\boldsymbol{x}-\boldsymbol{x}_{l}\right)=\mathbf{0} .
\end{aligned}
$$

Since $|\partial j|=\mathcal{O}(h)$, it proves the first line of (A.1). 


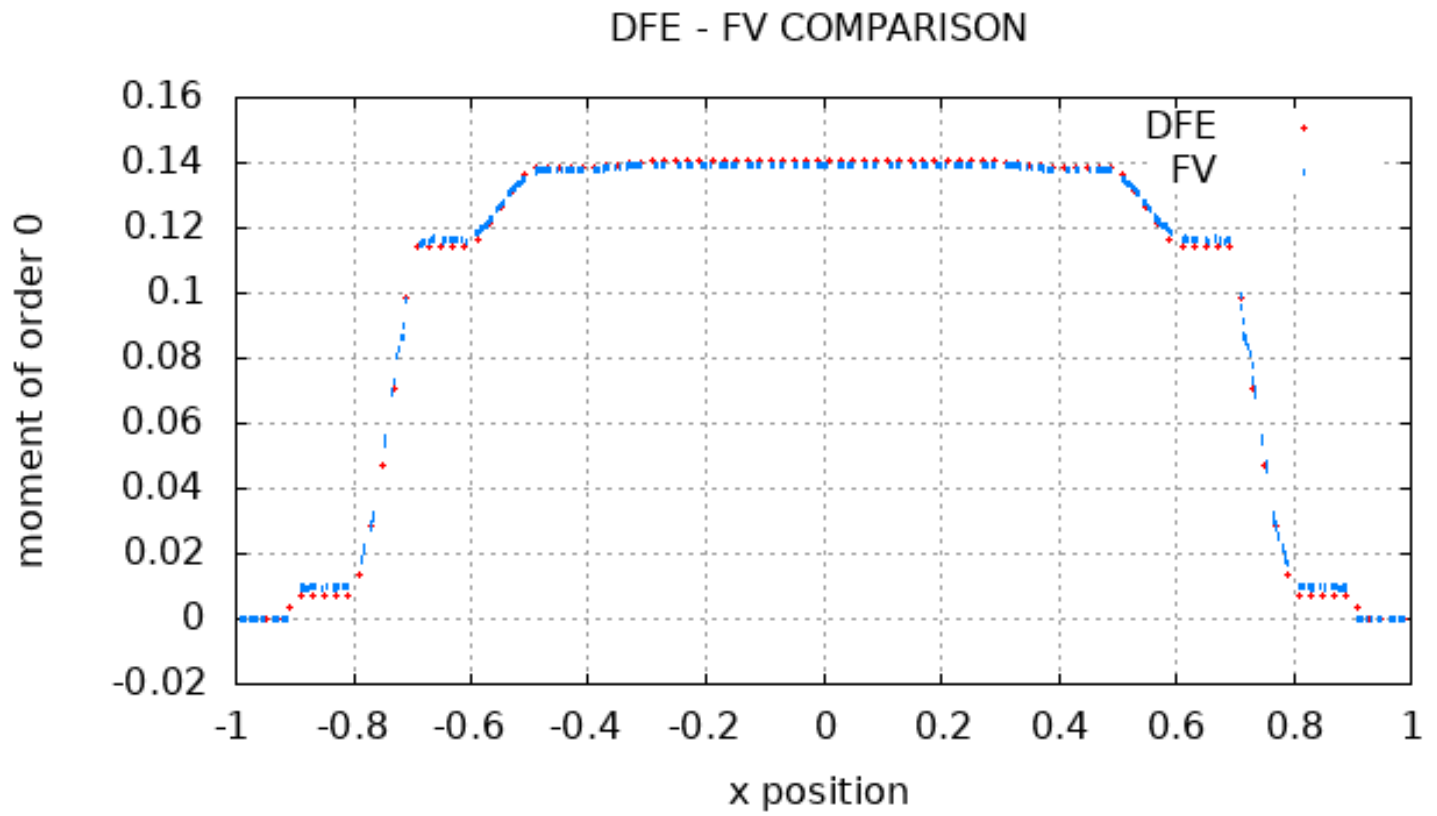

Figure 20: Comparison of the angular moment $e$ between finite volume and DFE, on unstructured mesh, for the transport/diffusion interfaces problem, with $N=11(K=484)$ and $\Delta t=1$, plot at time $t=100$.

Now, we define $l(r(j))$ as the edges sharing the vertex $r$ in the cell $j$ and $r(l(j))$ as the vertices of the edge $l$ of the cell $j$. Using $\mathbf{C}_{j r}=\frac{1}{2} \sum_{l(r(j))}|l| \boldsymbol{n}_{j l}$, and $\varphi_{l}=$ $\frac{1}{2} \sum_{r(l(j))} \varphi_{r}+\mathcal{O}\left(h^{2}\right)$, we deduce

$$
\begin{aligned}
\sum_{r(j)} \varphi_{r} \mathbf{C}_{j r} & =\frac{1}{2} \sum_{r(j)} \sum_{l(r(j))} \varphi_{r}|l| \mathbf{n}_{j l}, \\
& =\frac{1}{2} \sum_{l(j)} \sum_{r(l(j))} \varphi_{r}|l| \mathbf{n}_{j l}, \\
& =\sum_{l(j)}|l| \varphi_{l} \boldsymbol{n}_{j l}+\mathcal{O}\left(h^{3}\right) .
\end{aligned}
$$


mesh test 4

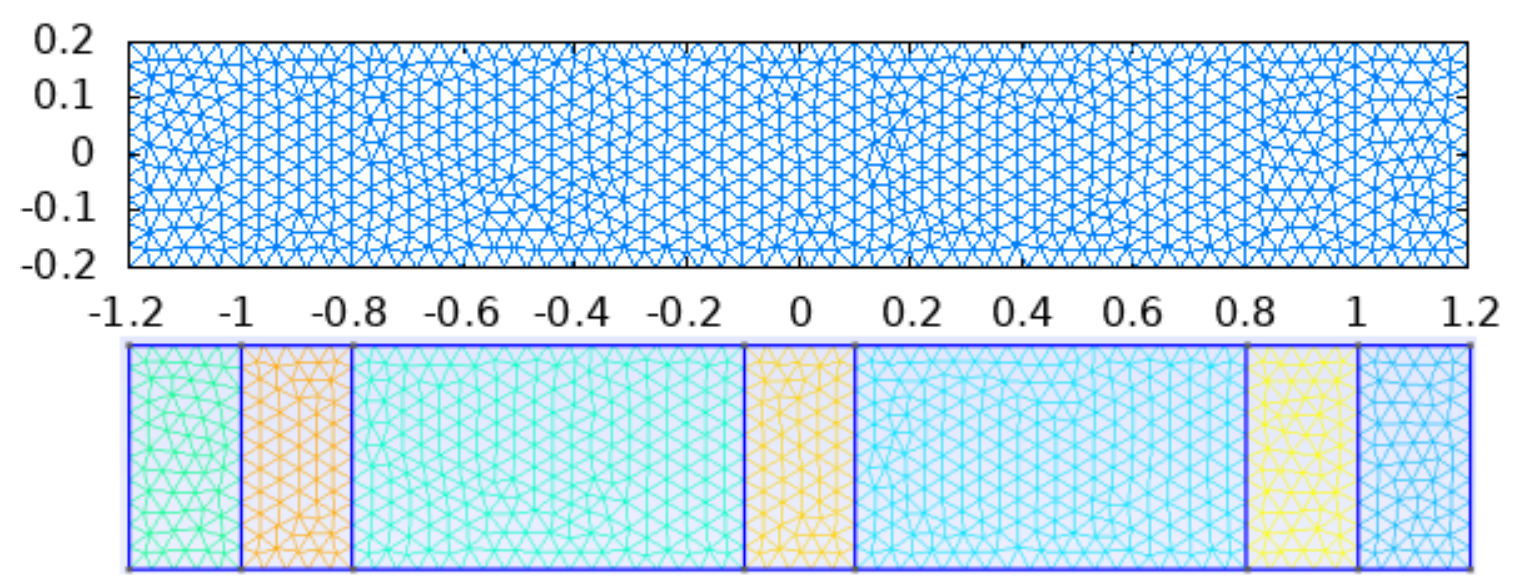

Figure 21: Unstructured mesh used for test four: stationary boundary layer.

Using $|\partial j|=\mathcal{O}(h)$, yields the second line of (A.1).

\section{Appendix B. Proof that $\overline{\bar{D}}_{r}^{n}$ is symmetric definite positive}

Due to the definition of $\overline{\overline{\boldsymbol{D}}}_{r}^{n}=v \Delta t \sum_{k=1}^{K} w_{k} a_{k, r}^{n} \boldsymbol{\omega}_{k} \otimes \boldsymbol{\omega}_{k}$, it is obvious that it is symmetric and non negative. The only non obvious property is the invertibility of the tensor. Let $\boldsymbol{x} \in \mathbb{R}^{d}$ ( $d$ the dimension of the space), we have to prove that $\boldsymbol{x}^{T} \overline{\overline{\boldsymbol{D}}}_{r}^{n} \boldsymbol{x}=0 \Leftrightarrow \boldsymbol{x}=\mathbf{0}$. The sufficient part of the condition is obvious. Simple algebra shows that

$$
\boldsymbol{x}^{T} v \Delta t \sum_{k=1}^{K} w_{k} a_{k, r}^{n} \boldsymbol{\omega}_{k} \otimes \boldsymbol{\omega}_{k} \boldsymbol{x}=v \Delta t \sum_{k=1}^{K} w_{k} a_{k, r}^{n}\left(\boldsymbol{\omega}_{k} \cdot \boldsymbol{x}\right)^{2} .
$$

Since $\Delta t, v, w_{k}$ and $a_{k, r}$ are positive and non zero, the condition $\boldsymbol{x}^{T} \overline{\overline{\boldsymbol{D}}}_{r}^{n} \boldsymbol{x}=0$ is equivalent to

$$
\boldsymbol{\omega}_{k} \cdot \boldsymbol{x}=0, \quad \forall k .
$$

Since there is at least $d$ independent vectors $\boldsymbol{\omega}_{k}$, the set of $\boldsymbol{\omega}_{\boldsymbol{k}}$ spans $\mathbb{R}^{d}$, meaning that $\boldsymbol{x}$ is orthogonal to all vectors of $\mathbb{R}^{d}$. So $\boldsymbol{x}=\mathbf{0}$. 


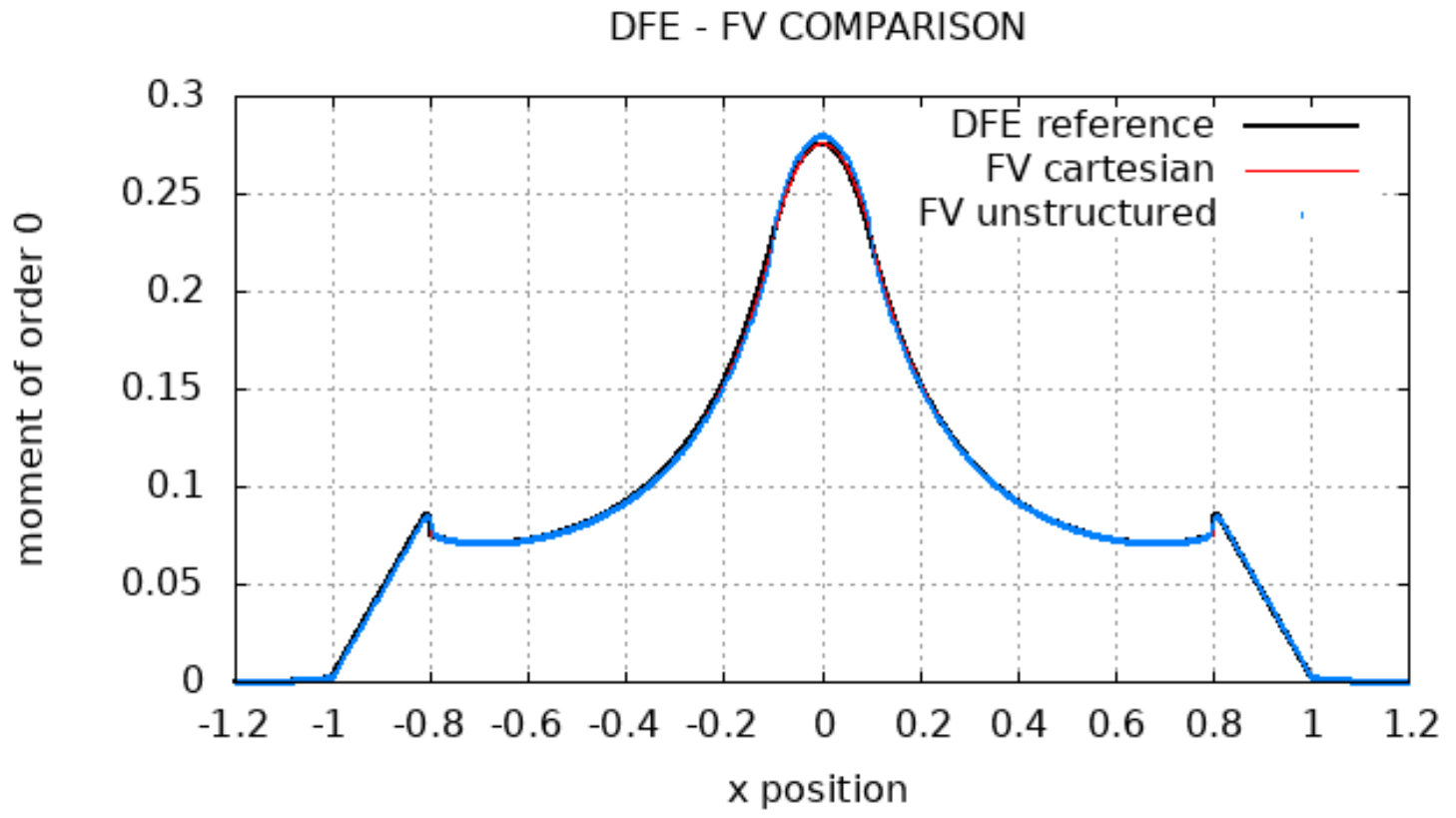

Figure 22: Comparison of the angular moment $e$ between the DFE method and the FV method in Cartesian and unstructured meshes, for the stationary boundary layer problem, with $N=4$ $(K=64)$ and $\Delta t=10^{9}$. The DFE reference uses 10000 cells (20000 degrees of freedom).

\section{Appendix C. Reminder about the Sherman-Morrison lemma}

A corollary of the Sherman-Morrison lemma. Let $\overline{\overline{\mathbf{I}}}_{K}$ be the identity matrix of size $K \times K$, and let $\boldsymbol{a}$ and $\boldsymbol{b}$ be two vectors of size $K$. The matrix $\overline{\mathbf{I}}_{K}-\boldsymbol{a} \otimes \boldsymbol{b}$ is invertible if $\boldsymbol{a} \cdot \boldsymbol{b} \neq 1$, and its inverse is:

$$
\left(\overline{\overline{\mathbf{I}}}_{K}-\boldsymbol{a} \otimes \boldsymbol{b}\right)^{-1}=\overline{\overline{\mathbf{I}}}_{K}+\frac{\boldsymbol{a} \otimes \boldsymbol{b}}{1-\boldsymbol{a} \cdot \boldsymbol{b}} .
$$




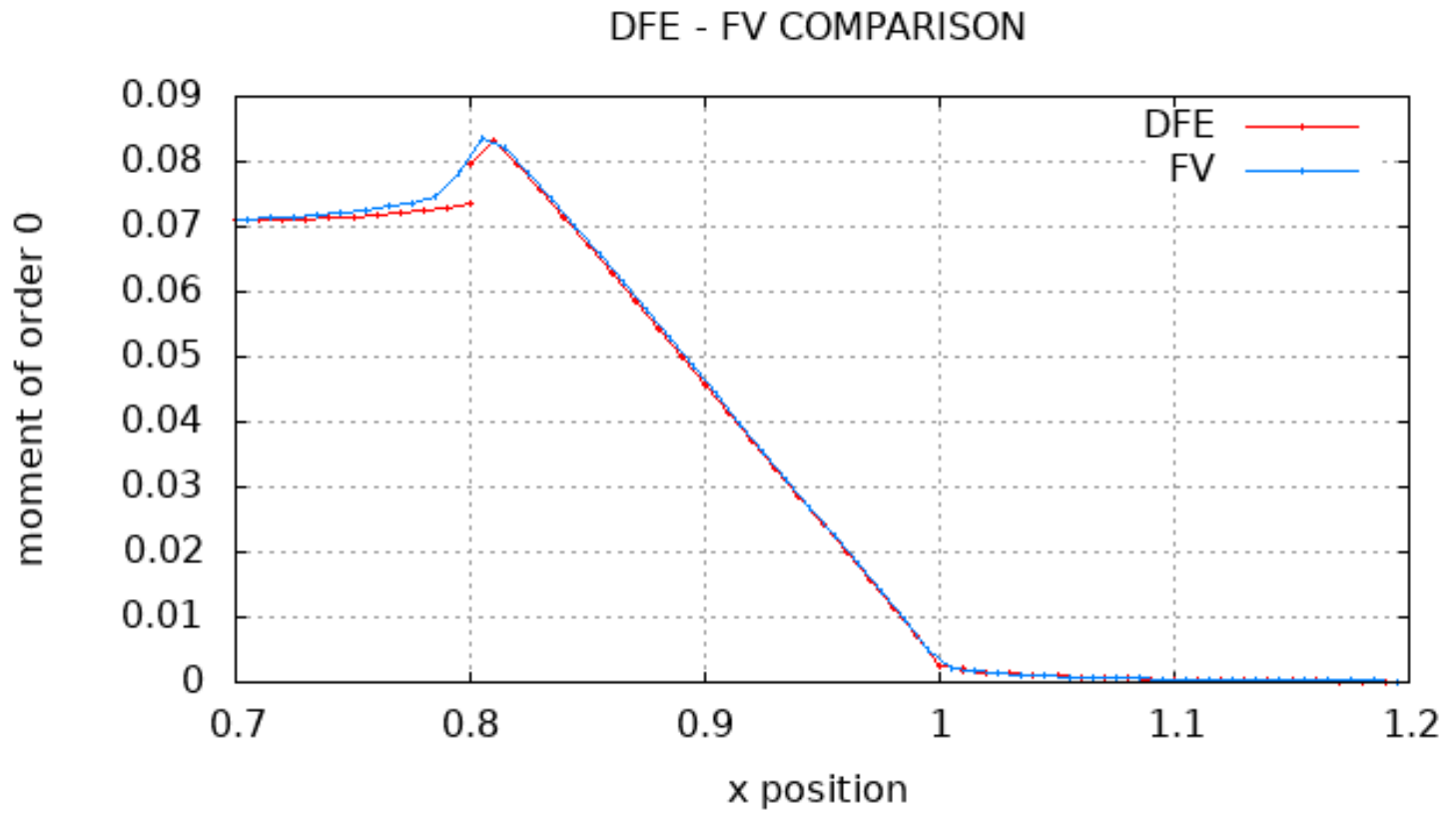

Figure 23: Comparison of the angular moment $e$ between finite volume and DFE, on Cartesian mesh, for the stationary boundary layer problem, with $N=4(K=64)$ and $\Delta t=10^{9}$. Zoom around $x=0.8$.

$\underline{\text { Proof: }}$

We start by noting that $(\boldsymbol{a} \otimes \boldsymbol{b})(\boldsymbol{a} \otimes \boldsymbol{b})=(\boldsymbol{a} \cdot \boldsymbol{b})(\boldsymbol{a} \otimes \boldsymbol{b})$; indeed:

For $i, j$ from 1 to $K:[(\boldsymbol{a} \otimes \boldsymbol{b})(\boldsymbol{a} \otimes \boldsymbol{b})]_{i, j}=\sum_{k=1}^{K} a_{i} b_{k} a_{k} b_{j}=(\boldsymbol{a} \cdot \boldsymbol{b})[(\boldsymbol{a} \otimes \boldsymbol{b})]_{i, j}$.

Then, we suppose that $\boldsymbol{a} \cdot \boldsymbol{b} \neq 1$ and we develop the product of matrices:

$$
\left(\overline{\overline{\mathbf{I}}}_{K}-\boldsymbol{a} \otimes \boldsymbol{b}\right)\left(\overline{\overline{\mathbf{I}}}_{K}+\frac{\boldsymbol{a} \otimes \boldsymbol{b}}{1-\boldsymbol{a} \cdot \boldsymbol{b}}\right)=\overline{\overline{\mathbf{I}}}_{K}-\boldsymbol{a} \otimes \boldsymbol{b}+\frac{\boldsymbol{a} \otimes \boldsymbol{b}}{1-\boldsymbol{a} \cdot \boldsymbol{b}}-\frac{(\boldsymbol{a} \otimes \boldsymbol{b})(\boldsymbol{a} \otimes \boldsymbol{b})}{1-\boldsymbol{a} \cdot \boldsymbol{b}}=\overline{\overline{\mathbf{I}}}_{K}
$$

that proves the corollary 


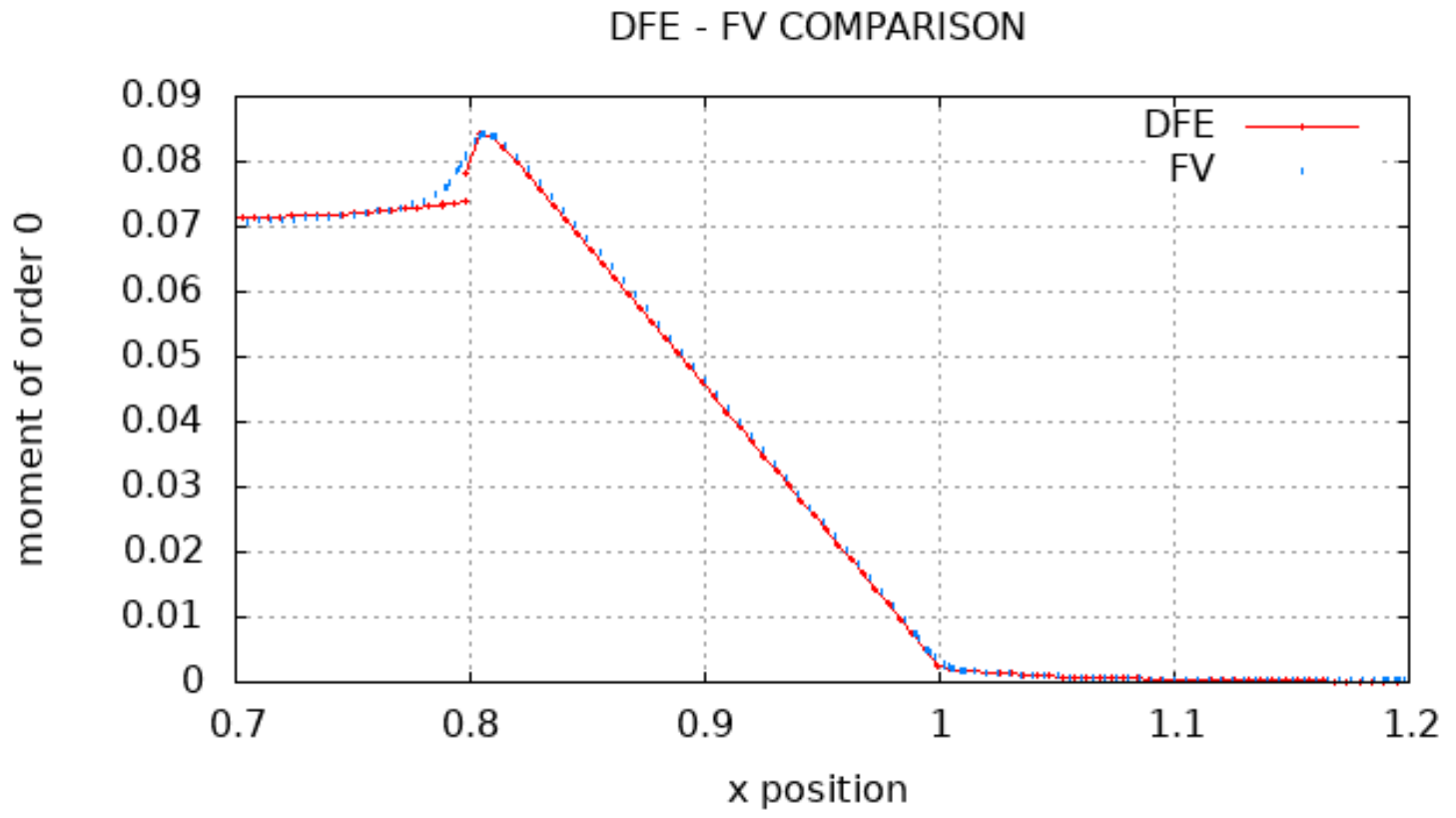

Figure 24: Comparison of the angular moment $e$ between finite volume and DFE, on unstructured mesh, for the stationary boundary layer problem, with $N=4(K=64)$ and $\Delta t=10^{9}$. Zoom around $x=0.8$.

\section{Appendix D. Proof that the advection scheme is conservative}

At convergence of the sub-iterations, we have an advection scheme of the form:

$$
\frac{\delta u_{k, r}^{n+1}-\delta u_{k, r}^{n}}{v \Delta t}+\left[\boldsymbol{\omega}_{k} \cdot \boldsymbol{\nabla} \delta u_{k}\right]_{r}^{n+1}=0,
$$

with

$$
\left[\boldsymbol{\omega}_{k} \cdot \boldsymbol{\nabla} \delta u_{k}\right]_{r}^{n+1}=\frac{1}{V_{r}} \sum_{\tilde{l}, \boldsymbol{n}_{r i} \cdot \boldsymbol{\omega}_{k}>0}|\tilde{\mid}| \boldsymbol{n}_{r \tilde{l}} \cdot \boldsymbol{\omega}_{\boldsymbol{k}} \delta u_{k, r}^{n+r}+\frac{1}{V_{r}} \sum_{\tilde{l}, \boldsymbol{n}_{r \tilde{l}} \boldsymbol{\omega}_{k}<0}|\tilde{l}| \boldsymbol{n}_{r \tilde{l}} \cdot \boldsymbol{\omega}_{\boldsymbol{k}} \delta u_{k, r^{\prime}(r)}^{n+1},
$$

where $\tilde{l}$ is the edge intersecting segment $\left[r r^{\prime}\right]$. 


\section{FV CONVERGENCE}

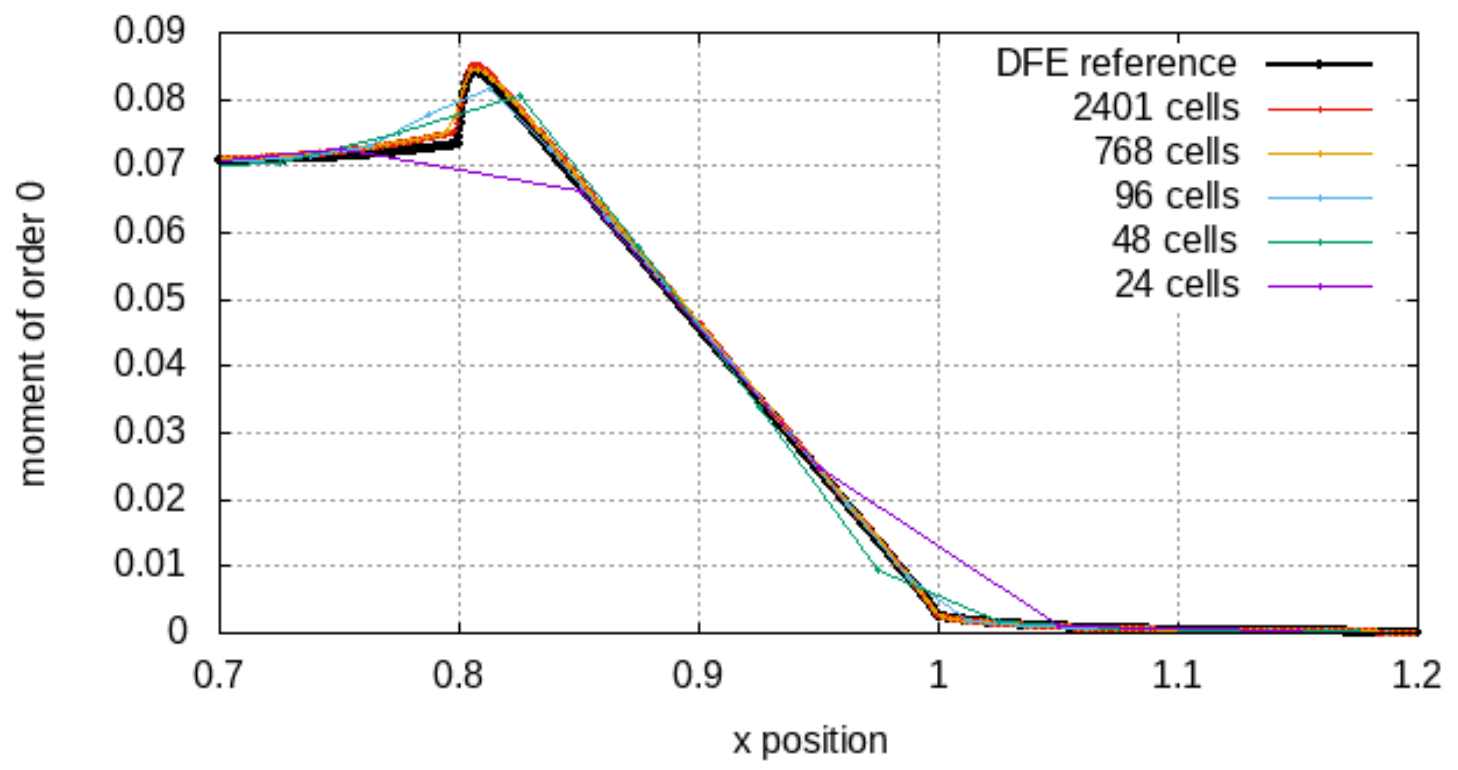

Figure 25: Comparison of the angular moment $e$ of the FV code, for different numbers of cells, for test 4: stationary boundary layer, with $N=4(K=64)$ and $\Delta t=10^{9}$. The DFE reference use 10000 cells (20000 degrees of freedom). Zoom around $x=0.8$.

We would like to show that the sum of the flux $\left[\boldsymbol{\omega}_{k} \cdot \boldsymbol{\nabla} \delta u_{k}\right]_{r}^{n+1}$ on each node is equal to zero. It remains to show that the value $V_{r} \delta u_{k, r}$ is conserved.

In the following, we consider periodic boundary conditions.

Let $r$ and $r^{\prime}$ be two adjacent nodes.

We write the flux from node $r$ to node $r^{\prime}$, we get

$$
F_{r r^{\prime}}= \begin{cases}|\tilde{l}| \boldsymbol{n}_{r \tilde{l}} \cdot \boldsymbol{\omega}_{\boldsymbol{k}} \delta u_{k, r}^{n+1} & \text { if } \boldsymbol{n}_{r \tilde{l}} \cdot \boldsymbol{\omega}_{\boldsymbol{k}}>0 \\ |\tilde{l}| \boldsymbol{n}_{r \tilde{l}} \cdot \boldsymbol{\omega}_{\boldsymbol{k}} \delta u_{k, r^{\prime}}^{n+1} & \text { if } \boldsymbol{n}_{r \tilde{l}} \cdot \boldsymbol{\omega}_{\boldsymbol{k}}<0\end{cases}
$$

Thus, $F_{r r^{\prime}}+F_{r^{\prime} r}=0$. 


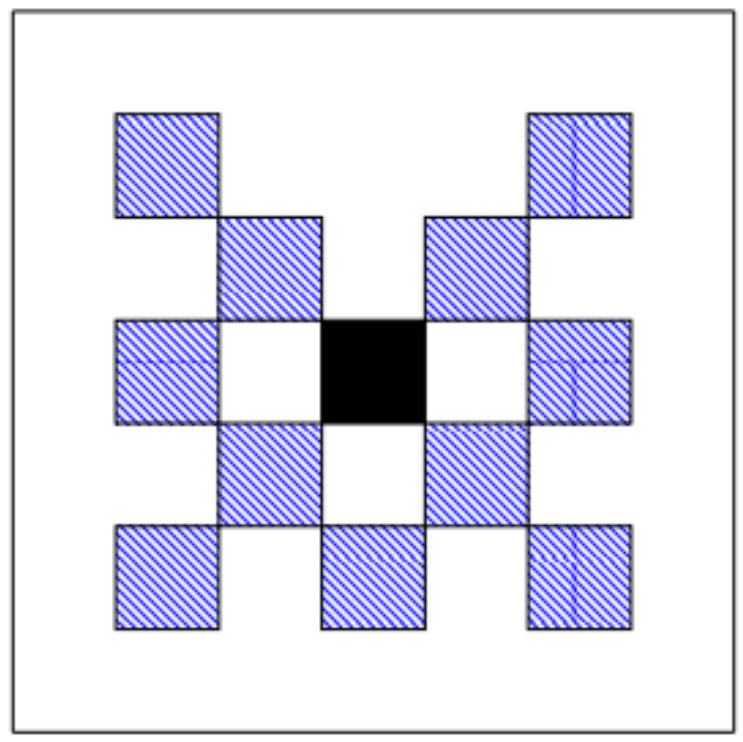

Figure 26: Lattice calculation domain.

\section{Appendix E. Proof that the advection scheme is unconditionally stable}

The advection scheme has the form

$$
\delta u_{k, r}^{n+1, p+1}=\delta u_{k, r}^{n}-\Delta t v\left[\boldsymbol{\omega}_{k} \cdot \boldsymbol{\nabla} \delta u_{k}\right]_{r}^{n+1, p+1 / 2} .
$$

We can rewrite it with the expression of $\left[\boldsymbol{\omega}_{k} \cdot \boldsymbol{\nabla} \delta u_{k}\right]_{r}^{n+1, p+1 / 2}$, which gives

$$
\delta u_{k, r}^{n+1, p+1}=\delta u_{k, r}^{n}-\Delta t v\left(\frac{1}{V_{r}} \sum_{\tilde{l}, \boldsymbol{n}_{r i} \boldsymbol{\omega}_{k}>0}|\tilde{l}| \boldsymbol{n}_{r \tilde{l}} \cdot \boldsymbol{\omega}_{\boldsymbol{k}} \delta u_{k, r}^{n+1, p+1}+\frac{1}{V_{r}} \sum_{\tilde{l}, \boldsymbol{n}_{r \tilde{l}} \cdot \boldsymbol{\omega}_{k}<0}|\tilde{l}| \boldsymbol{n}_{r \tilde{l}} \cdot \boldsymbol{\omega}_{\boldsymbol{k}} \delta u_{k, r^{\prime}(r)}^{n+1, p}\right) .
$$

$$
\text { Let } \nu_{r}=\frac{v \Delta t}{V_{r}}|\tilde{l}| \boldsymbol{n}_{r \tilde{l}} \cdot \boldsymbol{\omega}_{\boldsymbol{k}} \text { and } \nu=\frac{1}{V_{r}} \sum_{\tilde{l}, \boldsymbol{n}_{r i} \cdot \boldsymbol{\omega}_{k}>0} v \Delta t|\tilde{l}| \boldsymbol{n}_{r \tilde{l}} \cdot \boldsymbol{\omega}_{\boldsymbol{k}}=\sum_{\tilde{l}, \boldsymbol{n}_{r i} \cdot \boldsymbol{\omega}_{k}>0} \nu_{r} \text {. }
$$

Then, factorising by $\delta u_{k, r}^{n+1, p+1}$, we get

$$
\delta u_{k, r}^{n+1, p+1}(1+\nu)=\delta u_{k, r}^{n}-\sum_{\tilde{l}, \boldsymbol{n}_{r} \cdot \boldsymbol{\omega}_{k}<0} \nu_{r} \delta u_{k, r}^{n+1, p}
$$



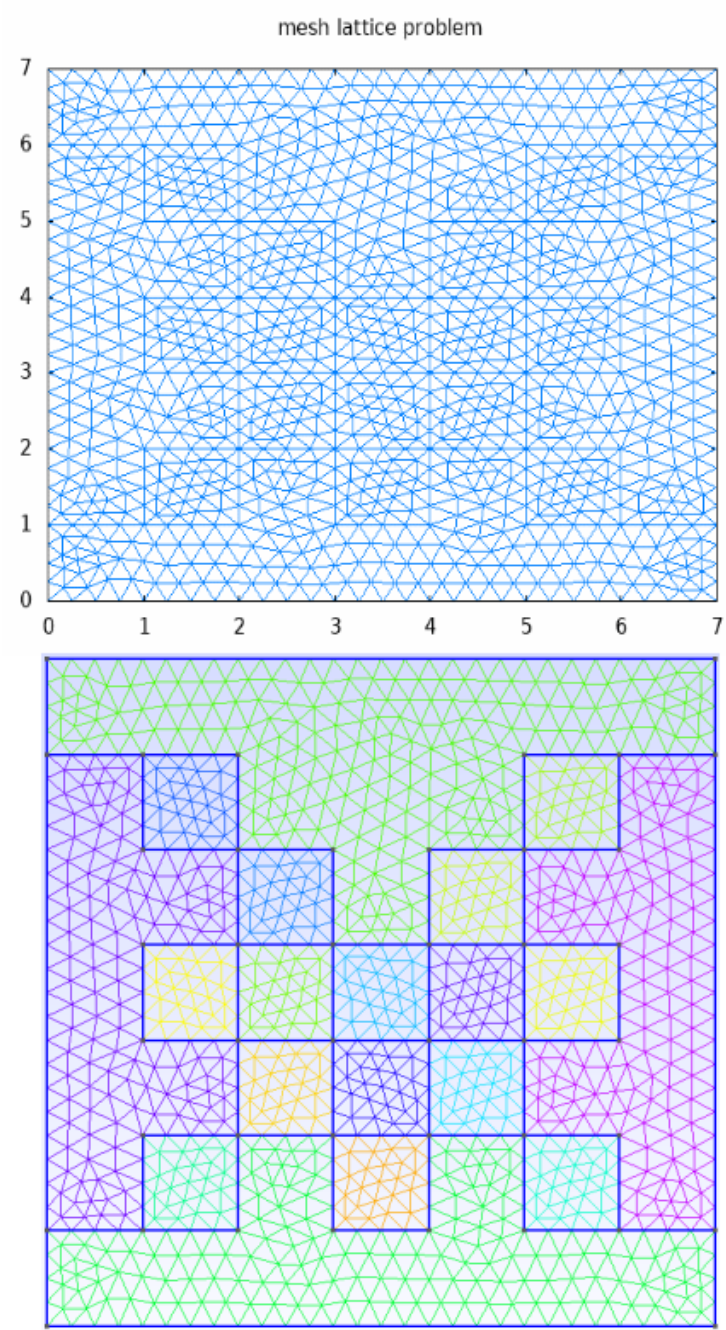

Figure 27: Unstructured mesh used for lattice problem.

$$
\Rightarrow \delta u_{k, r}^{n+1, p+1}=\frac{1}{1+\nu} \delta u_{k, r}^{n}-\sum_{\tilde{l}, \boldsymbol{n}_{r i} \boldsymbol{\omega}_{k}<0} \frac{\nu_{r}}{1+\nu} \delta u_{k, r^{\prime}}^{n+1, p} .
$$

Given that $\frac{1}{1+\nu}-\sum_{\tilde{l}, \boldsymbol{n}_{r i} \cdot \boldsymbol{\omega}_{k}<0} \frac{\nu_{r}}{1+\nu}=1$, we obtain a convex combination of $\delta u_{k, r}^{p+1, p+1}$ depending on $\delta u_{k, r}^{n}$ and $\delta u_{k, r^{\prime}}^{n+1, p}, \forall \nu$, which means $\forall \Delta t$. 


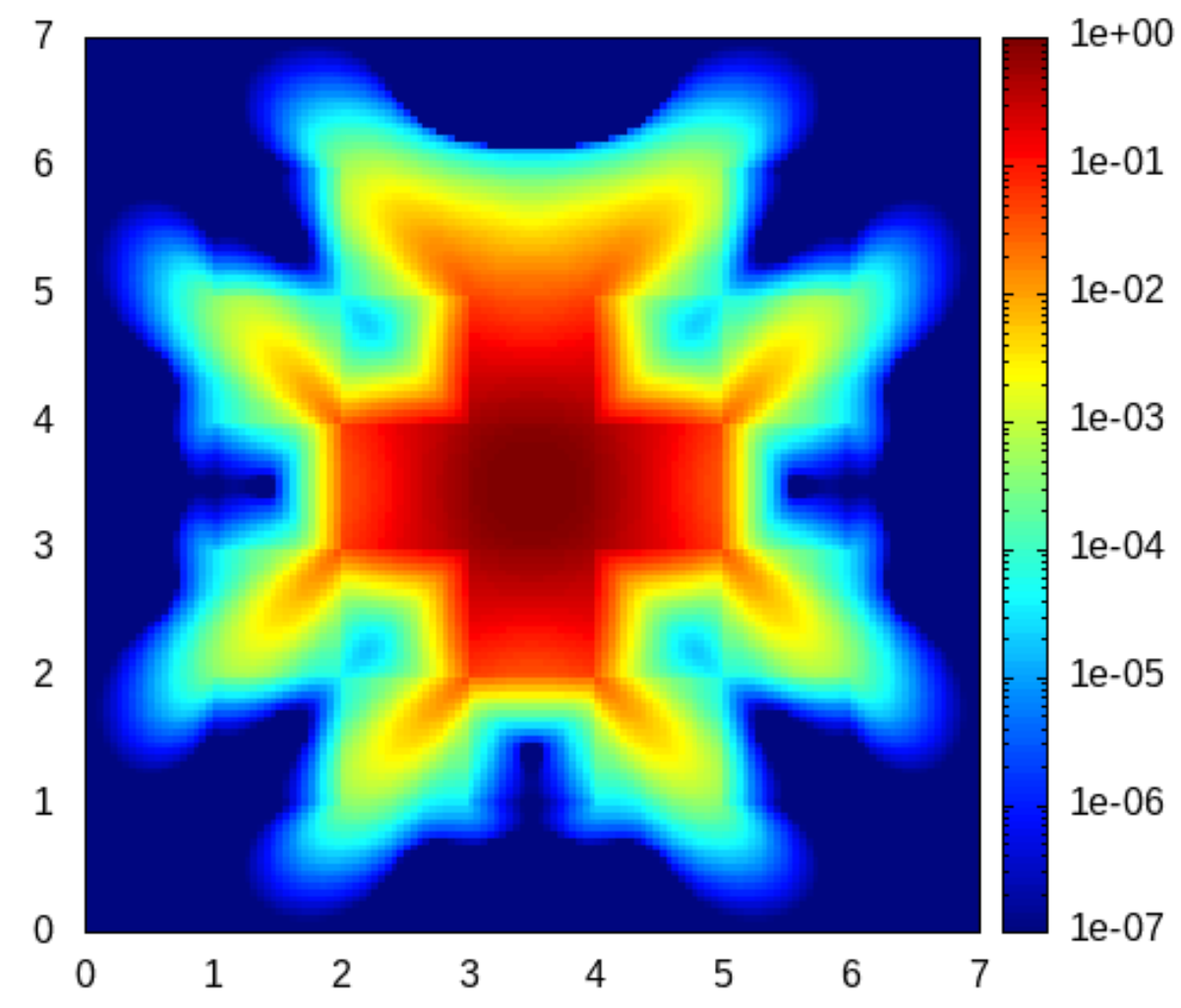

Figure 28: Cartesian mesh $140 \times 140$, lattice problem, map of $e, N=1(K=4)$, time $t=3.2$.

\section{Appendix F. Proof that the diffusion scheme is conservative}

We consider our diffusion scheme: $\left\{\begin{array}{l}\frac{1}{\Delta t}\left(e^{n+1}-e^{n}\right)+M\left(e^{n+1}\right) e^{n+1}=h+g \\ e^{n+1} \geq 0\end{array}\right.$ With

$$
\begin{aligned}
\Phi_{j, l} & =\mu_{1}|l|\left(\frac{\lambda_{j}}{\left\|\boldsymbol{x}_{j r_{1}}\right\|}+\frac{\eta_{j}}{\left\|\boldsymbol{x}_{j r_{2}}\right\|}\right), \\
\Phi_{m, l} & =\mu_{2}|l|\left(\frac{\lambda_{m}}{\left\|\boldsymbol{x}_{m r_{3}}\right\|}+\frac{\eta_{m}}{\left\|\boldsymbol{x}_{m r_{4}}\right\|}\right) .
\end{aligned}
$$




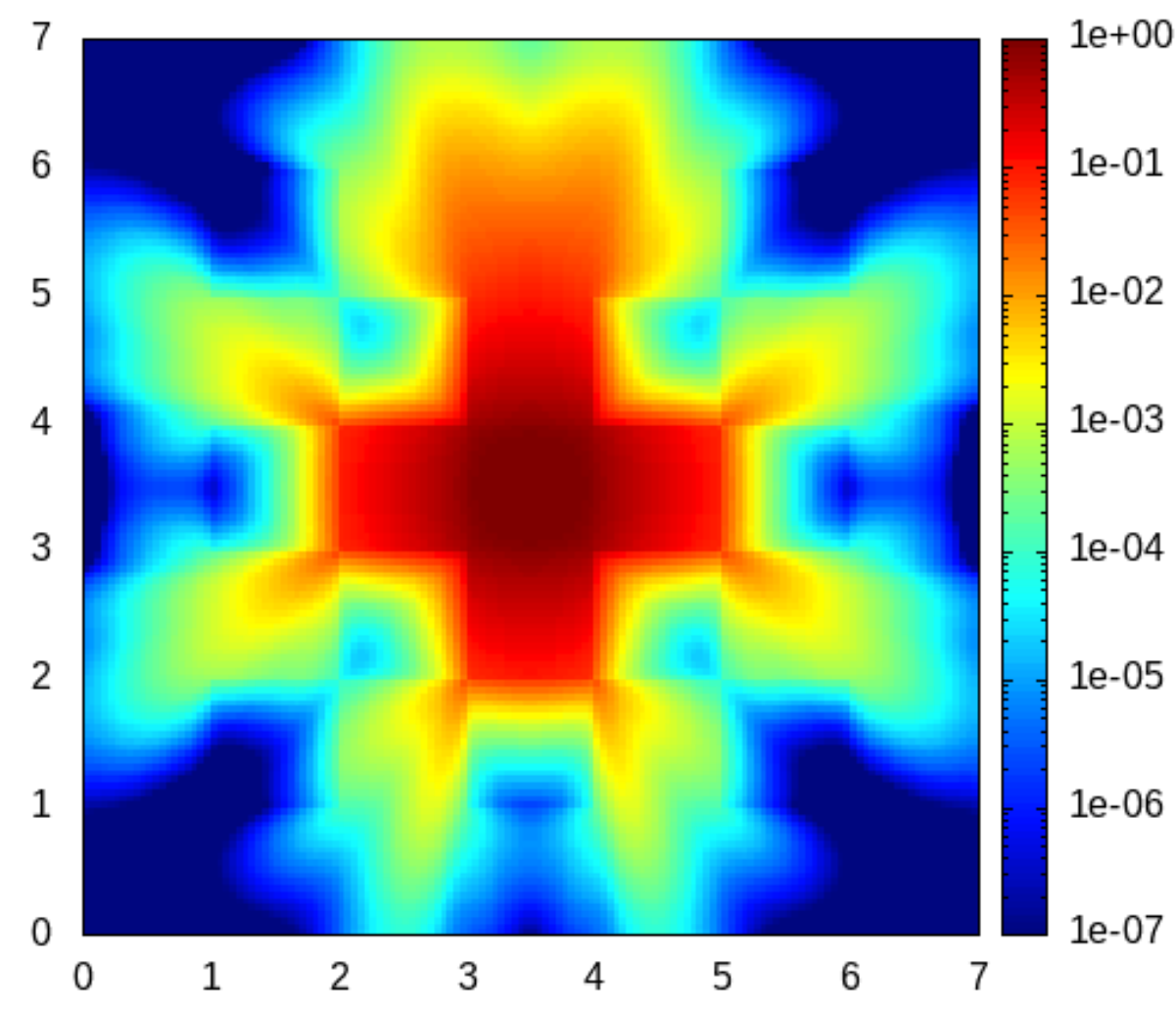

Figure 29: Cartesian mesh $140 \times 140$, lattice problem, map of $e, N=2(K=16)$, time $t=3.2$.

and

$$
\left\{\begin{array}{l}
{[M(e)]_{j j}=\frac{1}{V_{j}}\left(\sum_{l \in j \backslash \partial \Omega} \Phi_{j, l}+\sum_{l \in j \cap \partial \Omega}|l| \alpha_{j}\right)} \\
{[M(e)]_{j m}=-\frac{\Phi_{m, l}}{V_{j}} \text { if } j \neq m}
\end{array}\right.
$$

We consider periodic boundary conditions, so we have $[M(e)]_{j j}=\frac{1}{V_{j}}\left(\sum_{l \in j} \Phi_{j, l}\right)$.

We need to prove that if $h=0$, then $\forall n \geq 0, \sum_{j \in \mathcal{M}} V_{j} e_{j}^{n+1}=\sum_{j \in \mathcal{M}} V_{j} e_{j}^{n}$, where $V_{j}$ corresponds to the volume of cell $j$.

The continuous version of it is $\frac{d}{d t} \int_{\Omega} e=0$. 


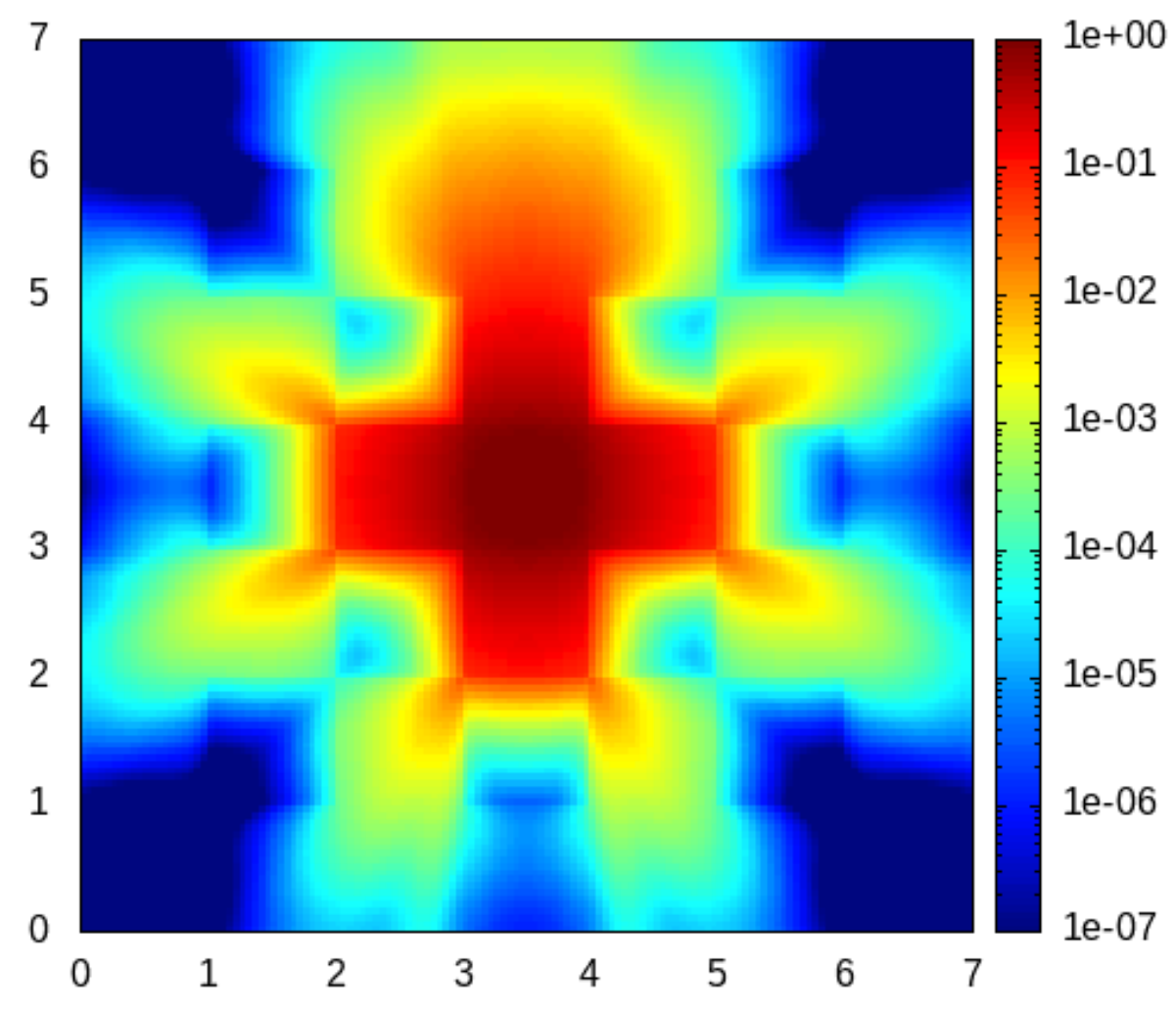

Figure 30: Cartesian mesh $140 \times 140$, lattice problem, map of $e, N=3(K=36)$, time $t=3.2$.

$$
\begin{gathered}
\forall j \in \mathcal{M}, \quad \frac{1}{\Delta t}\left(e_{j}^{n+1}-e_{j}^{n}\right)+\sum_{m \in \mathcal{M}}\left[M\left(e^{n+1}\right)\right]_{j m} e_{m}^{n+1}=0, \\
\Rightarrow \sum_{j \in \mathcal{M}} V_{j} e_{j}^{n+1}-\sum_{j \in \mathcal{M}} V_{j} e_{j}^{n}+\Delta t \sum_{j \in \mathcal{M}} V_{j} \sum_{m \in \mathcal{M}}\left[M\left(e^{n+1}\right)\right]_{j m} e_{m}^{n+1}=0 .
\end{gathered}
$$

And,

$$
\begin{gathered}
\sum_{j \in \mathcal{M}} V_{j} \sum_{m \in \mathcal{M}}\left[M\left(e^{n+1}\right)\right]_{j m} e_{m}^{n+1}=V_{j} \sum_{m \in \mathcal{M}}\left[M\left(e^{n+1}\right)\right]_{m m} e_{m}^{n+1}+\sum_{j \in \mathcal{M}, j \neq m} V_{j} \sum_{m \in \mathcal{M}}\left[M\left(e^{n+1}\right)\right]_{j m} e_{m}^{n+1} \\
=V_{j} \sum_{m \in \mathcal{M}} \sum_{l \in m} \frac{\Phi_{m, l}}{V_{j}} e_{m}^{n+1}+\sum_{j \in \mathcal{M}, j \neq m} V_{j} \sum_{m \in \mathcal{M}}-\frac{\Phi_{m, l}}{V_{j}} e_{m}^{n+1} \\
=\sum_{m \in \mathcal{M}} \sum_{l \in m} \Phi_{m, l} e_{m}^{n+1}-\sum_{m \in \mathcal{M}} \sum_{j \in \mathcal{M}, j \neq m} \Phi_{m, l} e_{m}^{n+1}
\end{gathered}
$$




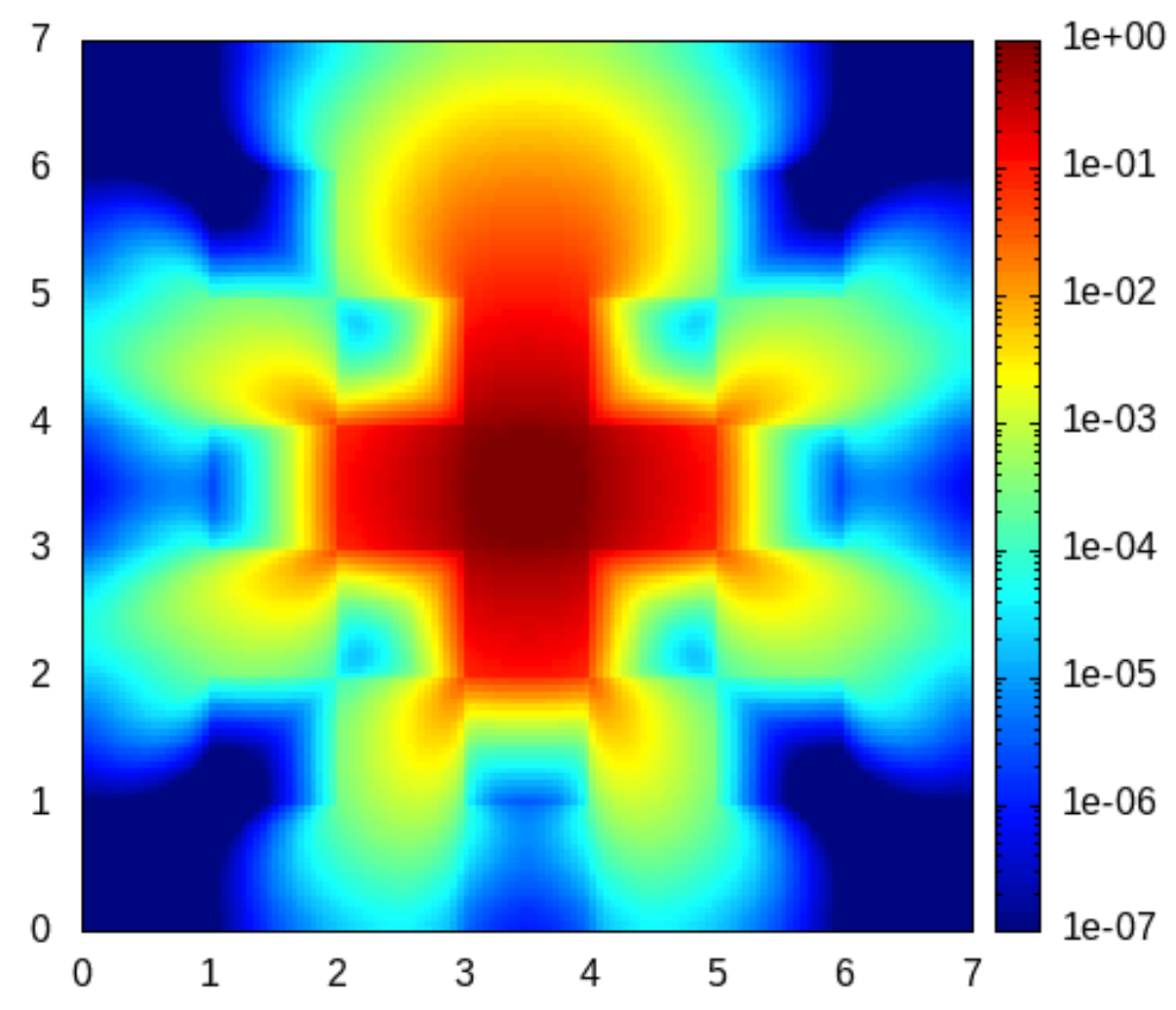

Figure 31: Cartesian mesh $140 \times 140$, lattice problem, map of $e, N=6(K=144)$, time $t=3.2$.

$$
=\sum_{m \in \mathcal{M}} \sum_{l \in m} \Phi_{m, l} e_{m}^{n+1}-\sum_{m \in \mathcal{M}} \sum_{l \in m} \Phi_{m, l} e_{m}^{n+1},
$$

${ }_{540}$ because $[M(e)]_{j m}=\Phi_{m, l}=0$ if $j$ and $m$ are not adjacent. Then, $\sum_{j \in \mathcal{M}} V_{j} \sum_{m \in \mathcal{M}}\left[M\left(e^{n+1}\right)\right]_{j m} e_{m}^{n+1}=0$, which implies:

$$
\forall n \geq 0, \quad \sum_{j \in \mathcal{M}} V_{j} e_{j}^{n+1}=\sum_{j \in \mathcal{M}} V_{j} e_{j}^{n}
$$




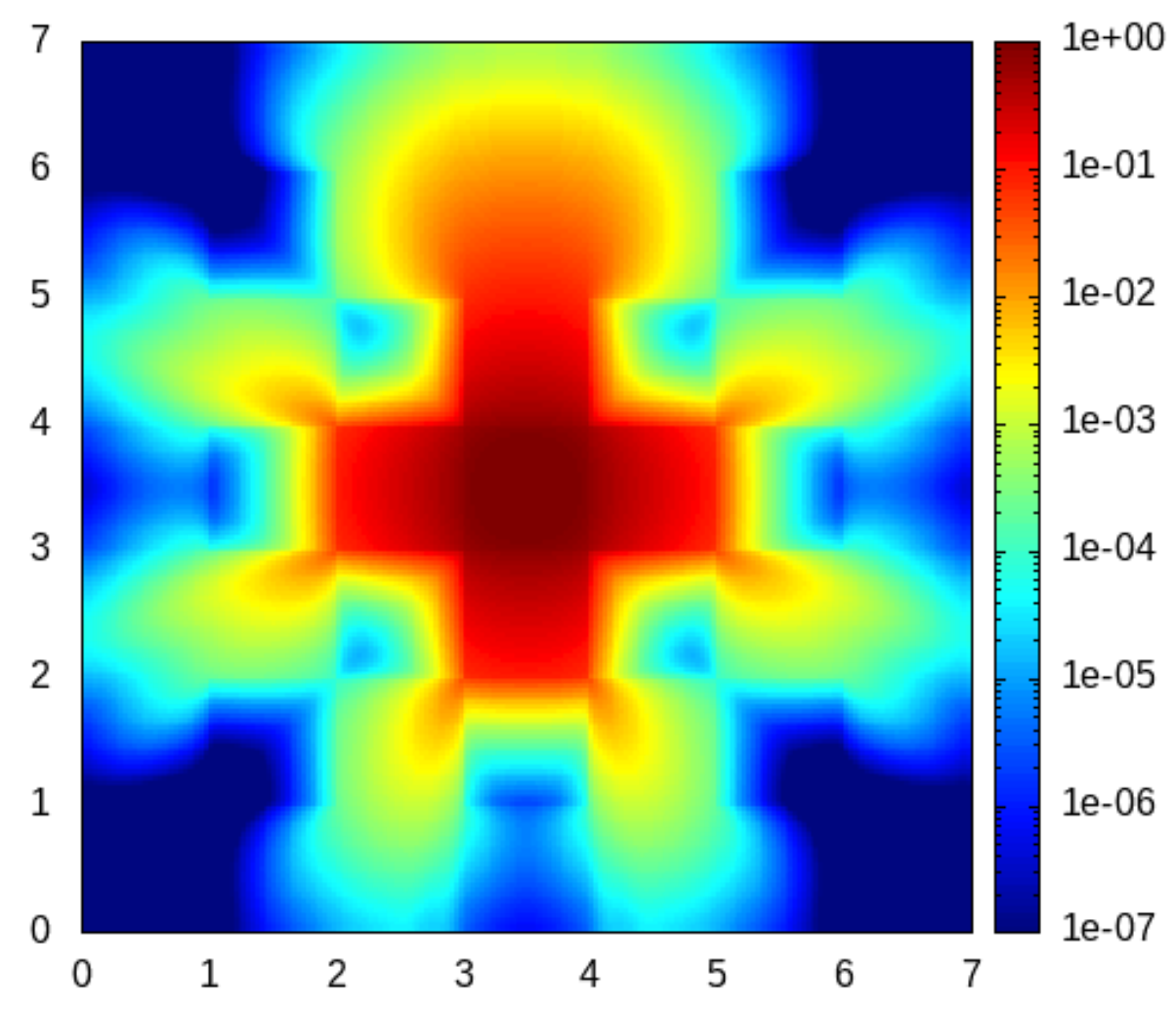

Figure 32: Cartesian mesh $210 \times 210$, lattice problem, map of $e, N=6(K=144)$, time $t=3.2$.

Appendix G. Proof that the diffusion scheme respect positivity and is unconditionally stable

Positivity. Setting $h=g=0$, our diffusion scheme writes: $e^{n+1}=e^{n}-\Delta t M\left(e^{n+1}\right) e^{n+1}$, with

$$
\begin{gathered}
\Phi_{j, l}=\mu_{1}|l|\left(\frac{\lambda_{j}}{\left\|\boldsymbol{x}_{j r_{1}}\right\|}+\frac{\eta_{j}}{\left\|\boldsymbol{x}_{j r_{2}}\right\|}\right), \\
\Phi_{m, l}=\mu_{2}|l|\left(\frac{\lambda_{m}}{\left\|\boldsymbol{x}_{m r_{3}}\right\|}+\frac{\eta_{m}}{\left\|\boldsymbol{x}_{m r_{4}}\right\|}\right) .
\end{gathered}
$$

and

$$
\left\{\begin{array}{l}
{[M(e)]_{j j}=\frac{1}{V_{j}}\left(\sum_{l \in j \backslash \partial \Omega} \Phi_{j, l}+\sum_{l \in j \cap \partial \Omega}|l| \alpha_{j}\right)} \\
{[M(e)]_{j m}=-\frac{\Phi_{m, l}}{V_{j}} \text { if } j \neq m}
\end{array}\right.
$$




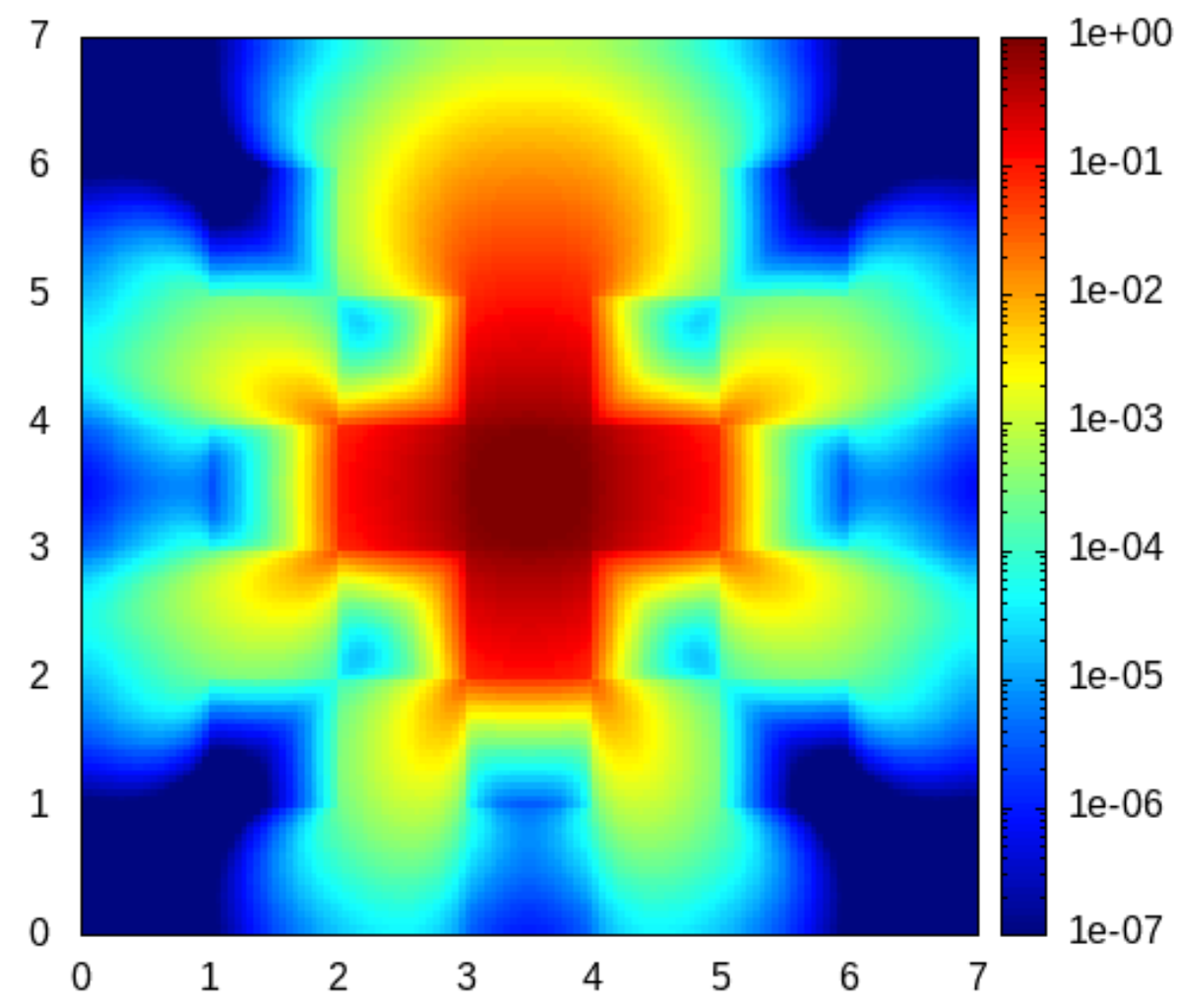

Figure 33: Cartesian mesh $140 \times 140$, lattice problem, map of $e, N=8(K=256)$, time $t=3.2$.

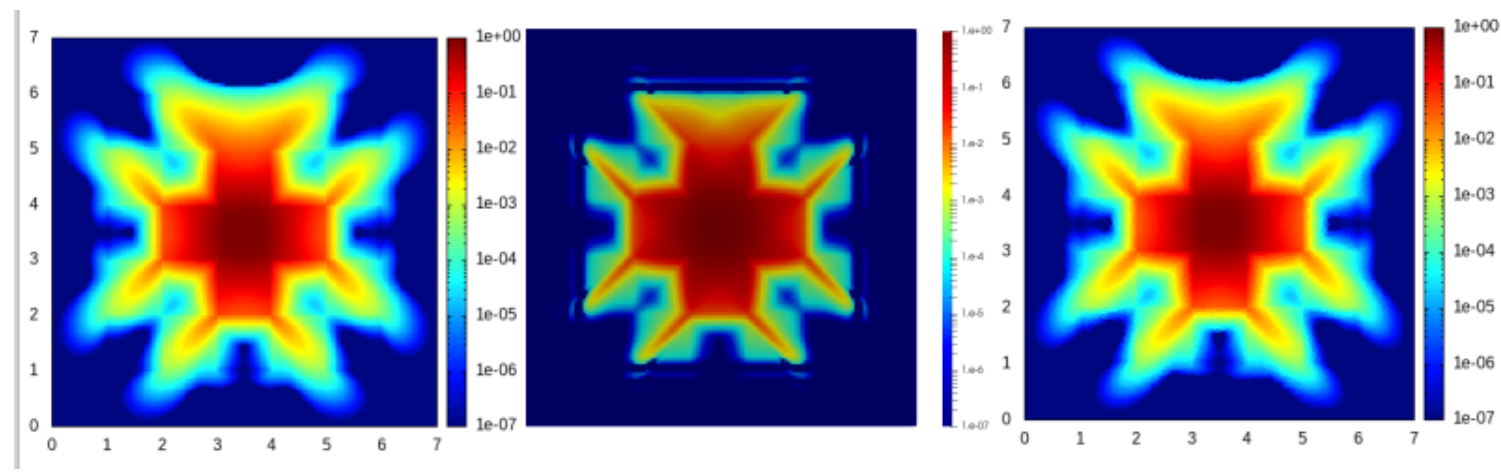

Figure 34: Comparison of results on lattice problem. Left: FV method, Cartesian mesh $140 \times 140$. Middle: DFE code, Cartesian mesh $140 \times 140$. Right: FV method unstructured mesh. $K=4$, time $t=3.2$.

We denote by $S$ the set of the index of the cells. Then, for all $j \in S$, we have

$$
e_{j}^{n+1}=e_{j}^{n}-\Delta t \sum_{m \in M}[M(e)]_{j m} e_{m}^{n+1}
$$



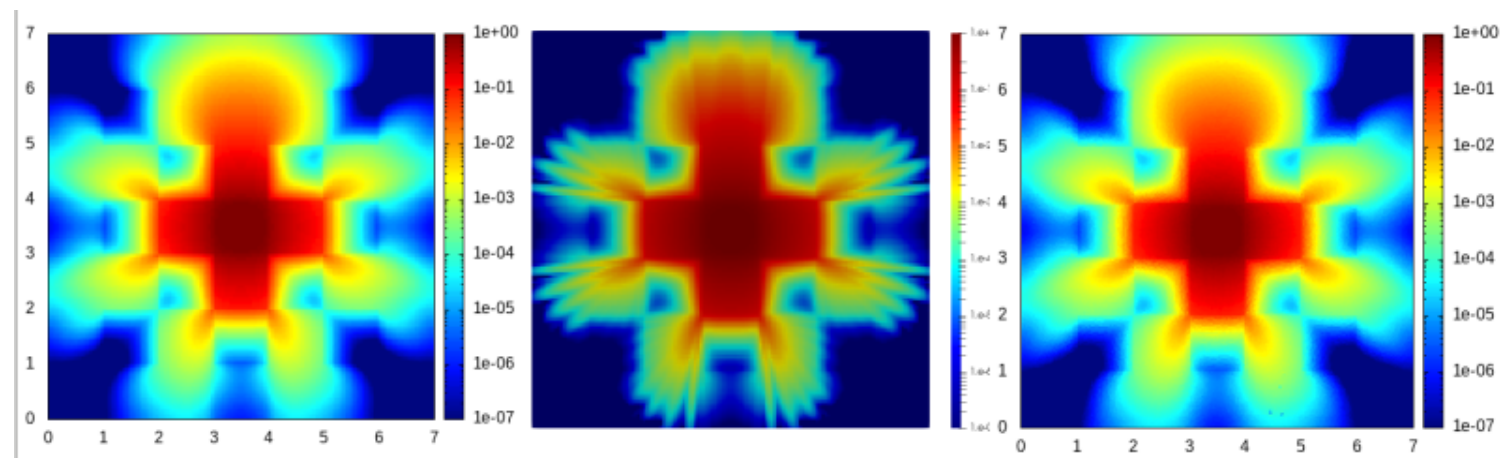

Figure 35: Comparison of results on lattice problem. Left: FV method, Cartesian mesh $140 \times 140$. Middle: DFE code, Cartesian mesh $140 \times 140$. Right: FV method unstructured mesh. $K=144$, time $t=3.2$.

$$
\begin{aligned}
& \Rightarrow e_{j}^{n+1}=e_{j}^{n}-\Delta t[M(e)]_{j j} e_{j}^{n+1}-\Delta t \sum_{m \in S, m \neq j}[M(e)]_{j m} e_{m}^{n+1} \\
& \Rightarrow e_{j}^{n+1}=\frac{1}{1+\Delta t[M(e)]_{j j}} e_{j}^{n}-\sum_{m \in S, m \neq j} \frac{\Delta t[M(e)]_{j m}}{1+\Delta t[M(e)]_{j j}} e_{m}^{n+1}
\end{aligned}
$$

Given definition of $[M(e)]_{j j}$ and $[M(e)]_{j m}$, we are able to easily show that $\frac{1}{1+\Delta t[M(e)]_{j j}}$ and $\sum_{m \in S, m \neq j} \frac{-\Delta t[M(e)]_{j m}}{1+\Delta t[M(e)]_{j j}}$ are two positive quantities.

Thus, the diffusion scheme is positive.

Stability. Concerning the stability of the scheme, we refer to [14].

In this article, it is shown that for $h \geq 0, g \geq 0$ and $e^{n} \geq 0$, it exists a solution $e^{n+1}$, and the fixed point strategy converges to it.

\section{Appendix H. Proof that the global scheme is conservative}

Summing over all the cells Eq. (25), considering the absorption term $\sigma_{a}=0$ and the source $s=0$, one gets

$$
\begin{aligned}
& \sum_{j} V_{j} e_{j}^{n+1, p+1}-\sum_{j} v \Delta t \sum_{l(j)}|l|\left(\overline{\overline{\boldsymbol{D}}}_{l}^{n}[\boldsymbol{\nabla} e]_{l}^{n+1, p+1}\right) \cdot \boldsymbol{n}_{j l} \\
= & \sum_{j} V_{j} e_{j}^{n}-\sum_{j} v \Delta t \sum_{r(j)} \sum_{k=1}^{K} w_{k} \boldsymbol{\omega}_{k}\left(\alpha_{k, r}^{n}+\beta_{k, r}^{n+1, p}\right) \cdot \boldsymbol{C}_{j r},
\end{aligned}
$$


The term $\sum_{j} v \Delta t \sum_{l(j)}|l|\left(\overline{\bar{D}}_{l}^{n}[\nabla e]_{l}^{n+1, p+1}\right) \cdot \boldsymbol{n}_{j l}=0$ because of property 3.6.

$$
\begin{aligned}
\sum_{j} \sum_{r(j)} \sum_{k=1}^{K} w_{k} \boldsymbol{\omega}_{k}\left(\alpha_{k, r}^{n}+\beta_{k, r}^{n+1, p}\right) \cdot \boldsymbol{C}_{j r} & =\sum_{r} \sum_{k=1}^{K} \sum_{j(r)} w_{k} \boldsymbol{\omega}_{k}\left(\alpha_{k, r}^{n}+\beta_{k, r}^{n+1, p}\right) \cdot \boldsymbol{C}_{j r} \\
& =\sum_{r} \sum_{k=1}^{K} w_{k} \boldsymbol{\omega}_{k}\left(\alpha_{k, r}^{n}+\beta_{k, r}^{n+1, p}\right) \cdot \sum_{j(r)} \boldsymbol{C}_{j r} \\
& =0,
\end{aligned}
$$

because $\sum_{j(r)} \boldsymbol{C}_{j r}=\mathbf{0}$, refer for instance to [22].

Appendix I. Proof that $\sum_{k=1}^{K} w_{k} \delta u_{k}^{n}=0, \quad \forall n$.

We prove it by induction. Initially, $\sum_{k=1}^{K} \delta u_{k, r}^{0}=0$. Let us assume that $\sum_{k=1}^{K} w_{k} \delta u_{k, r}^{n+1, p}=$ 0 .

We consider the scheme:

$$
\begin{aligned}
& \frac{1}{v} \frac{\delta u_{k, r}^{n+1, p+1}-\delta u_{k, r}^{n}}{\Delta t}+\left[\boldsymbol{\omega}_{k} \cdot \boldsymbol{\nabla} \delta u_{k}\right]_{r}^{n+1, p+1 / 2}+\sigma_{t, r}^{n} \delta u_{k, r}^{n+1, p+1} \\
= & \sum_{k^{\prime}=1}^{K} w_{k^{\prime}}\left[\boldsymbol{\omega}_{k^{\prime}} \cdot \boldsymbol{\nabla} \delta u_{k^{\prime}}\right]_{r}^{n+1, p+1 / 2}-\boldsymbol{\omega}_{k} \cdot[\boldsymbol{\nabla} e]_{r}^{n+1, p+1}+\delta q_{k, r}^{n}
\end{aligned}
$$

Then, we multiply the equation by $w_{k}$ and we sum over $\mathrm{k}$.

Using that $\sum_{k} w_{k}=1$, we have $\sum_{k=1}^{K} w_{k}\left[\boldsymbol{\omega}_{k} \cdot \nabla \delta u_{k}\right]_{r}^{n+1, p+1 / 2}=\sum_{k=1}^{K} w_{k} \sum_{k^{\prime}=1}^{K} w_{k^{\prime}}\left[\boldsymbol{\omega}_{k^{\prime}} \cdot \boldsymbol{\nabla} \delta u_{k^{\prime}}\right]_{r}^{n+1, p+1 / 2}$.

Then, we have $\sum_{k=1}^{K} w_{k} \delta q_{k, r}^{n}=\sum_{k=1}^{K} w_{k} q_{k, r}^{n}-\sum_{k=1}^{K} w_{k} s_{r}^{n}=s_{r}^{n}-s_{r}^{n}=0$, by definition of $\delta q$.

Then, we have that $\sum_{k=1}^{K} w_{k} \boldsymbol{\omega}_{k} \cdot[\boldsymbol{\nabla} e]_{r}^{n+1, p+1}=[\boldsymbol{\nabla} e]_{r}^{n+1, p+1} \cdot \sum_{k=1}^{K} w_{k} \boldsymbol{\omega}_{k}=0$ because 
$\sum_{k=1}^{K} w_{k} \boldsymbol{\omega}_{k}=0$, by definition.

$$
\begin{gathered}
\text { Finally, we get }\left(\frac{1}{v \Delta t}+\sigma_{t, r}^{n}\right) \sum_{k=1}^{K} w_{k} \delta u_{k, r}^{n+1, p+1}=\frac{1}{v \Delta t} \sum_{k=1}^{K} w_{k} \delta u_{k, r}^{n}=0 \\
\Rightarrow \sum_{k=1}^{K} w_{k} \delta u_{k, r}^{n+1, p+1}=0
\end{gathered}
$$

because $\frac{1}{v \Delta t}+\sigma_{t, r}^{n} \neq 0$. Then by induction, $\sum_{k=1}^{K} w_{k} \delta u_{k, r}^{m, q}=0 \quad \forall m$ and $\forall q$.

\section{Appendix J. Proof that the global scheme is Asymptotic Preserving}

From the hypothesis $\sigma_{s} \rightarrow \infty, \sigma_{a} \rightarrow 0$ and $q \rightarrow 0$, up to $\mathcal{O}\left(\frac{1}{\sigma_{s}^{2}}\right)$, we define the discrete Knudsen number $\varepsilon=1 /\left(v \Delta t \sigma_{t, r}^{n}\right)$. In addition, we assume that the initial condition is well-prepared, meaning that $\delta u_{k, r}^{n}=\mathcal{O}(\varepsilon)$.

Then from definition (17), we infer that:

$$
a_{k, r}^{n}=\varepsilon+\mathcal{O}\left(\varepsilon^{2}\right), \quad \text { and } \quad b_{k, r}^{n}=\mathcal{O}(1) .
$$

It yields that $\left(\overline{\overline{\mathbf{I}}}_{K}+\frac{\boldsymbol{a}_{r}^{n} \otimes \boldsymbol{b}_{r}^{n}}{1-\boldsymbol{a}_{r}^{n} \cdot \boldsymbol{b}_{r}^{n}}\right)=\overline{\overline{\mathbf{I}}}_{K}+\mathcal{O}(\varepsilon)$ and from Eq. (18) that

$$
\mathcal{A}_{k, r}^{n}=\mathcal{O}\left(\varepsilon^{2}\right), \quad \mathcal{B}_{k, r}^{n+1}=\mathcal{O}\left(\varepsilon^{2}\right) \text { and } \mathcal{C}_{k, r}^{n+1}=\mathcal{O}(\varepsilon) .
$$

We can now infer from Eq. (19) and Eq. (20) that

$$
\delta u_{k, r}^{n+1}=\mathcal{O}(\varepsilon) .
$$

Moreover, the expression of the flux $\boldsymbol{f}_{r}^{n+1, p+1}$ given by Eq. (22) reduces to

$$
\boldsymbol{f}_{r}^{n+1, p+1}=-\overline{\overline{\boldsymbol{D}}}_{r}^{n}[\boldsymbol{\nabla} e]_{r}^{n+1, p+1}+\mathcal{O}\left(\varepsilon^{2}\right),
$$

and from the definition (23) of $\overline{\overline{\boldsymbol{D}}}_{r}^{n}$, we deduce that

$$
\begin{aligned}
\overline{\overline{\boldsymbol{D}}}_{r}^{n} & =\sum_{k=1}^{K} w_{k} \frac{v \Delta t}{v \Delta t \sigma_{t, r}^{n}} \boldsymbol{\omega}_{k} \otimes \boldsymbol{\omega}_{k}+\mathcal{O}\left(\varepsilon^{2}\right), \\
& =\frac{1}{3 \sigma_{t, r}^{n}} \overline{\overline{\mathbf{I}}}_{K}+\mathcal{O}\left(\varepsilon^{2}\right)
\end{aligned}
$$


because of the constraints on the angular quadrature (6). The reformulation (25) does not change this analysis, which implies the result.

\section{References}

[1] Adams, M. (1977). Subcell Balance Methods for Radiative Transfer on Arbitrary Grids. Transport Theory and Statistical Physics, 26:385-431.

[2] Adams, M. (2001). Discontinuous Finite Element Transport Solutions in Thick Diffusive Problems. Nuclear Science and Engineering, 137:298 - 333.

[3] Adams, M. L. and Nowak, P. F. (1998). Asymptotic analysis of a computational method for time- and frequency-dependent radiative transfer. Journal of Computational Physics, 146(1):366 - 403.

[4] Alcouffe, R. E. (1977). Diffusion synthetic acceleration methods for the diamond-differenced discrete-ordinates equations. Nuclear Science and Engineering, 64(2):344-355.

[5] Bailey, T. (2008). The piecewise linear discontinuous finite element method applied to the $R Z$ and $X Y Z$ transport equations. PhD thesis, Texas A\&M University.

[6] Bailey, T., Chang, J. H., Warsa, J. S., and Adams, M. L. (2011). A Piecewise Bi-Linear Discontinuous Finite Element Spatial Discretization of the Sn Transport Equation. In MEC 2011: International conference on mathematics and computational methods applied to nuclear science and engineering, Brazil.

[7] Bal, G. and Maday, Y. (2002). Coupling of transport and diffusion models in linear transport theory. ESAIM: Mathematical Modelling and Numerical AnalysisModélisation Mathématique et Analyse Numérique, 36(1):69-86.

[8] Bardos, C., Golse, F., Perthame, B., and Sentis, R. (1988). The nonaccretive radiative transfer equations: Existence of solutions and Rosseland approximation. J. Funct. Anal., 77(2):434-460.

[9] Bennoune, M., Lemou, M., and Mieussens, L. (2008). Uniformly stable numerical schemes for the Boltzmann equation preserving the compressible Navier-Stokes asymptotics. Journal of Computational Physics, 227(8):3781-3803. 
[10] Berthon, C., Moebs, G., Sarazin-Desbois, C., and Turpault, R. (2016). An asymptotic-preserving scheme for systems of conservation laws with source terms on 2D unstructured meshes. Communications in Applied Mathematics and Computational Science, 11(1):55-77.

[11] Berthon, C. and Turpault, R. (2011). Asymptotic preserving HLL schemes. Numerical methods for partial differential equations, 27(6):1396-1422.

[12] Blachère, F. and Turpault, R. (2016). An admissibility and asymptoticpreserving scheme for systems of conservation laws with source term on 2D unstructured meshes. Journal of Computational Physics, 315:98-123.

[13] Blachère, F. and Turpault, R. (2017). An admissibility and asymptotic preserving scheme for systems of conservation laws with source term on 2D unstructured meshes with high-order MOOD reconstruction. Computer Methods in Applied Mechanics and Engineering, 317:836-867.

[14] Blanc, X. and Labourasse, E. (2016). A positive scheme for diffusion problems on deformed meshes. ZAMM - Journal of Applied Mathematics and Mechanics / Zeitschrift für Angewandte Mathematik und Mechanik, 96(6):660-680.

[15] Brunner, T. A. and Holloway, J. P. (2005). Two-dimensional time dependent Riemann solvers for neutron transport. Journal of Computational Physics, 210(1):386 $-399$.

[16] Buet, C., Després, B., and Franck, E. (2015). Asymptotic preserving schemes on distorted meshes for Friedrichs systems with stiff relaxation: application to angular models in linear transport. Journal of Scientific Computing, 62(2):371-398.

[17] Buet, C., Després, B., Franck, E., and Leroy, T. (2017). Proof of uniform convergence for a cell-centered AP discretization of the hyperbolic heat equation on general meshes. Mathematics of Computation, 86(305):1147-1202.

[18] Buet, C., Despres, B., and Morel, G. (2020). Trefftz Discontinuous Galerkin basis functions for a class of Friedrichs systems coming from linear transport. Advances in Computational Mathematics, 46:1-27.

[19] Caramana, E. J., Burton, D. E., Shashkov, M. J., and Whalen, P. P. (1998). The construction of compatible hydrodynamics algorithms utilizing conservation of total energy. Journal of Computational Physics, 146:227-262. 
[20] Carlson, B. G. (1970). Transport theory: discrete ordinates quadrature over the unit sphere. Technical report, Los Alamos Scientific Lab., N. Mex.

[21] Carlson, B. G. and Lathrop, K. D. (1968). Computing Methods in Reactor Physics, page 171. Gordon and Breach.

[22] Carré, G., Del Pino, S., Després, B., and Labourasse, E. (2009). A cell-centered Lagrangian hydrodynamics scheme on general unstructured meshes in arbitrary dimension. Journal of Computational Physics, 228(14):5160-5183.

[23] Case, K. and Zweifel, P. (1967). Linear Transport Theory. Addison-Wesley series in nuclear engineering. Addison-Wesley Publishing Company.

[24] Castor, J. I. (2004). Radiation Hydrodynamics. Cambridge University Press.

[25] Chaland, F. and Samba, G. (2016). Discrete Ordinates Method for the Transport Equation Preserving One-Dimensional Spherical Symmetry in Two-Dimensional Cylindrical Geometry. Nuclear Science and Engineering, 182(4):417-434.

[26] Coudière, Y., Vila, J.-P., and Villedieu, P. (1999). Convergence rate of a finite volume scheme for a two dimensional convection-diffusion problem. Math. Model. Numer. Anal., 33(3):493-516.

[27] Crestetto, A., Crouseilles, N., Dimarco, G., and Lemou, M. (2019). Asymptotically complexity diminishing schemes (ACDS) for kinetic equations in the diffusive scaling. Journal of Computational Physics, 394:243 - 262.

[28] Crockatt, M., Christlieb, A., Garrett, K., and Hauck, C. (2017). An arbitraryorder, fully implicit, hybrid kinetic solver for linear radiative transport using integral deferred correction. Journal of Computational Physics, 346:212 - 241.

[29] Crouseilles, N. and Lemou, M. (2011). An asymptotic preserving scheme based on a micro-macro decomposition for collisional Vlasov equations: diffusion and high-field scaling limits. Kinetic and Related Models, 4(2):441-477.

[30] Dautray, R. and Lyons, J.-L. (1985). Analyse mathématique et calcul numérique pour les sciences et les techniques. Number 6. Masson.

[31] Degond, P., Dimarco, G., and Mieussens, L. (2010). A multiscale kinetic-fluid solver with dynamic localization of kinetic effects. Journal of Computational Physics, 229(13):4907 - 4933. 
[32] Degond, P., Jin, S., and Mieussens, L. (2005). A smooth transition model between kinetic and hydrodynamic equations. Journal of Computational Physics, 209(2):665-694.

[33] Del Pino, S., Labourasse, E., and Morel, G. (2018). An asymptotic preserving multidimensional ALE method for a system of two compressible flows coupled with friction. Journal of Computational Physics, 363:268 - 301.

[34] Einkemmer, L., Hu, J., and Wang, Y. (2020). An asymptotic-preserving dynamical low-rank method for the multi-scale multi-dimensional linear transport equation. arXiv preprint arXiv:2005.06571.

[35] Eymard, R., Gallouët, T., Guichard, C., Herbin, R., and Masson, R. (2014). TP or not TP, that is the question. Computational Geosciences, 18(3-4):285-296.

[36] Fleck Jr, J. A. and Cummings Jr, J. D. (1971). An implicit Monte Carlo scheme for calculating time and frequency dependent nonlinear radiation transport. Journal of Computational Physics, 8(3):313-342.

[37] Franck, E. (2012). Design and numerical analysis of asymptotic preserving schemes on unstructured meshes. Application to the linear transport and Friedrichs systems. PhD thesis, Université Pierre et Marie Curie.

[38] Golse, F., Jin, S., and Levermore, C. D. (2003). A domain decomposition analysis for a two-scale linear transport problem. ESAIM: Mathematical Modelling and Numerical Analysis, 37(6):869-892.

[39] Gosse, L. (2011). Transient radiative transfer in the grey case: Well-balanced and asymptotic-preserving schemes built on Case's elementary solutions. Journal of Quantitative Spectroscopy and Radiative Transfer, 112(12):1995-2012.

[40] Hauck, C. D. and McClarren, R. G. (2013). A collision-based hybrid method for time-dependent, linear, kinetic transport equations. Multiscale Modeling $\&$ Simulation, 11(4):1197-1227.

[41] Hermeline, F. (2000). A finite volume method for the approximation of diffusion operators on distorted meshes. Journal of Computational Physics, 160(2):481-499.

[42] Hermeline, F. (2016). A discretization of the multigroup PN radiative transfer equation on general meshes. Journal of Computational Physics, 313:549 - 582. 
[43] Himpe, C., Leibner, T., and Rave, S. (2018). Hierarchical approximate proper orthogonal decomposition. SIAM Journal on Scientific Computing, 40(5):A3267A3292.

[44] Hu, J., Jin, S., and Li, Q. (2017). Chapter 5 - Asymptotic-Preserving Schemes for Multiscale Hyperbolic and Kinetic Equations. In Abgrall, R. and Shu, C.-W., editors, Handbook of Numerical Methods for Hyperbolic Problems, volume 18 of Handbook of Numerical Analysis, pages 103 - 129. Elsevier.

[45] Jin, S. (2012). Asymptotic preserving (AP) schemes for multiscale kinetic and hyperbolic equations: a review. Rivista di Matematica della Universita di Parma, $3: 177-216$.

[46] Jin, S. and Levermore, C. (1996). Numerical schemes for hyperbolic conservation laws with stiff relaxation terms. Journal of Computational Physics, 126:449-467.

[47] Jin, S. and Shi, Y. (2010). A micro-macro decomposition-based asymptoticpreserving scheme for the multispecies boltzmann equation. SIAM Journal on Scientific Computing, 31(6):4580-4606.

[48] Klar, A. (1998). Asymptotic-induced domain decomposition methods for kinetic and drift diffusion semiconductor equations. SIAM Journal on Scientific Computing, 19(6):2032-2050.

[49] Klar, A. and Schmeiser, C. (2001). Numerical passage from radiative heat transfer to nonlinear diffusion models. Mathematical Models and Methods in Applied Sciences, 11(05):749-767.

[50] Laiu, M. P., Frank, M., and Hauck, C. D. (2019). A Positive AsymptoticPreserving Scheme for Linear Kinetic Transport Equations. SIAM Journal on Scientific Computing, 41(3):A1500-A1526.

[51] Larsen, E. and Morel, J. (1989). Asymptotic solutions of numerical transport problems in optically thick, diffusive regimes II. Journal of Computational Physics, 83(1):212 - 236 .

[52] Larsen, E., Morel, J., and Miller, W. (1987). Asymptotic solutions of numerical transport problems in optically thick, diffusive regimes. Journal of Computational Physics, 69(2):283 - 324 .

[53] Larsen, E. W. and Keller, J. B. (1974). Asymptotic solution of neutron transport problems for small mean free paths. Journal of Mathematical Physics, 15(1):75-81. 
[54] Lemou, M. and Mieussens, L. (2008). A New Asymptotic Preserving Scheme Based on Micro-Macro Formulation for Linear Kinetic Equations in the Diffusion Limit. SIAM Journal on Scientific Computing, 31(1):334-368.

[55] Lipnikov, K., Svyatskiy, D., and Vassilevski, Y. (2010). A monotone finite volume method for advection-diffusion equations on unstructured polygon meshes. Journal of Computational Physics, 229(11):4017-4032.

[56] Maire, P.-H., Abgrall, R., Breil, J., and Ovadia, J. (2007). A cell-centered Lagrangian scheme for two-dimensional compressible flow problems. SIAM J. Sci. Comput., 29(4):1781-1824.

[57] Mazeran, C. (2007). Sur la structure mathématique et l'approximation numérique de l'hydrodynamique Lagrangienne bidimensionelle. PhD thesis, Université Bordeaux I.

[58] McClarren, R. and Hauck, C. (2010). Robust and accurate filtered spherical harmonics expansions for radiative transfer. Journal of Computational Physics, 229(16):5597 - 5614 .

[59] Mihalas, D. and Mihalas, B. W. (1984). Foundations of radiation hydrodynamics. New York etc.: Oxford University Press. XV, 718 p.

[60] Nikitin, K. and Vassilevski, Y. (2010). A monotone nonlinear finite volume method for advection-diffusion equations on unstructured polyhedral meshes in 3D. Russian J. Numer. Anal. Math. Modelling, 25(4):335-358.

[61] Plimpton, S., Hendrickson, B., Burns, S., and Rauchwerger, L. (2005). Parallel Sn sweeps on unstructured grids: Algorithms for prioritization, grid partitioning, and cycle detection. Nucl. Sci. Eng., 150(3):267-283.

[62] Radice, D., Abdikamalov, E., Rezzolla, L., and Ott, C. (2013). A new spherical harmonics scheme for multi-dimensional radiation transport I. Static matter configurations. Journal of Computational Physics, 242:648 - 669.

[63] Ramone, G., Adams, M., and Nowak, P. (1997). A Transport Synthetic Acceleration Method for Transport Iterations. Nuclear Science and Engineering, 125(3):257-283.

[64] Roger, M., Caliot, C., Crouseilles, N., and Coelho, P. (2014). A hybrid transportdiffusion model for radiative transfer in absorbing and scattering media. Journal of Computational Physics, 275:346 - 362. 
[65] Samba, G. (1986). Discontinuous Finite Element Method for Solving the TwoDimesional Neutron Transport Equation on Lagrangian Meshes. Nuclear Science and Engineering, 92(2):197-203.

[66] Schäfer, M., Frank, M., and Levermore, C. D. (2011). Diffusive corrections to $\$ p_{n}$ n\$ approximations. Multiscale Modeling \& Simulation, 9(1):1-28.

[67] Seibold, B. and Frank, M. (2014). StaRMAP-A Second Order Staggered Grid Method for Spherical Harmonics Moment Equations of Radiative Transfer. $A C M$ Trans. Math. Softw., 41(1).

[68] Sheng, Z. and Yuan, G. (2008). A finite volume scheme for diffusion equations on distorted quadrilateral meshes. Transport Theory Statist. Phys., 37(2-4):171-207.

[69] Toro, E. F. (1997). Riemann solvers and numerical methods for fluid dynamics. Springer.

[70] von Neumann, J. and Richtmyer, R. (1950). A method for the numerical calculation of hydrodynamic shocks. J. Appl. Phys., 21(3):232-237.

775 [71] Zel'dovich, Y. B. and Raizer, Y. P. (1966). Physics of Shock Waves and HighTemperature Hydrodynamic Phenomena. Academic Press. 